\title{
Strategies for Expanding Structural Diversity Available from Olefin Isomerization-Claisen Rearrangement Reactions
}

\author{
Supporting Information
}

\author{
Benjamin D. Stevens, Christopher J. Bungard and Scott G. Nelson* \\ Department of Chemistry, University of Pittsburgh, Pittsburgh, Pennsylvania 15260
}

TABLE OF CONTENTS

Compounds 7a-d

General Procedure A for Preparing Propargylic Ethers 5a-d

Compounds 5a-d

General Procedure B for Preparing Vinyl Boronic Esters 6a-d

Compounds 6a-d

General Procedure $\mathbf{C}$ for Preparing $\beta$-Boronic Aldehydes 8a-d

Compounds 8a-d, 11, 12, 14

General Procedure D for Suzuki Cross Coupling Reactions of 14

Compounds 15a-f

$\mathrm{X}$-ray structure data for compounds $\mathbf{8 c}$ and 12'

${ }^{1} \mathrm{H}$-NMR Study of $\mathrm{LiO}^{n} \mathrm{Pr}$ Addition to $\beta$-Boronic Aldehyde 8a

${ }^{1} \mathrm{H}$ and ${ }^{13} \mathrm{C}$ spectra
$\mathrm{S} 1-\mathrm{S} 2$

S2

S3

S3

S4

S5

S5-S8

S8

S8-S11

S12-S13

S14

S15-S68

General Information: Unless otherwise stated all reactions were performed in dry glassware under an atmosphere of oxygen free nitrogen using standard inert atmosphere techniques for the manipulation of solvents and reagents. Anhydrous solvents were obtained by passing through successive alumina columns on a solvent purification system. $\left[\mathrm{IrCl}\left(\mathrm{C}_{8} \mathrm{H}_{14}\right)_{2}\right]_{2}{ }^{1}$ and $\mathrm{PCy}_{3}$ were stored and weighed out in a glove box. Acetone was distilled from Drierite and stored under dry nitrogen. Pinacolborane was purchased from Aldrich, distilled under partial vacuum, and stored under nitrogen in a freezer. Chemical shifts are reported relative to residual $\mathrm{C}_{\mathbf{H}} \mathrm{Cl}_{3}$ (7.27 ppm) for ${ }^{1} \mathrm{H}$, and $\mathrm{CDCl}_{3}(77.0 \mathrm{ppm})$ or $\mathrm{CD}_{3} \mathrm{CN}(1.39 \mathrm{ppm})$ for ${ }^{13} \mathrm{C}$ NMR spectra. Reactions conducted using microwave irradiation were performed using a CEM Discover microwave reactor with internal temperature monitoring using an integrated infrared detector.

1-Phenyl-hept-1-en-3-ol (7a): ${ }^{2}$ To $1.90 \mathrm{~mL}(2.00 \mathrm{~g}, 15.1 \mathrm{mmol})$ of cinnamaldehyde in $15 \mathrm{~mL}$ of $\mathrm{Et}_{2} \mathrm{O}$ at $-78^{\circ} \mathrm{C}$ was added $10.4 \mathrm{~mL}(16.6 \mathrm{mmol})$ of $n$-butyl lithium in hexanes $(1.6 \mathrm{M})$ via syringe. The mixture was stirred at $-78{ }^{\circ} \mathrm{C}$ for $30 \mathrm{~min}$, then quenched carefully with sat. aq. $\mathrm{NH}_{4} \mathrm{Cl}$. The aqueous layer was extracted with $\mathrm{Et}_{2} \mathrm{O}(3 \mathrm{x})$, the combined organic layers were dried over $\mathrm{Na}_{2} \mathrm{SO}_{4}$, filtered and the crude product mixture was concentrated in vacuo. Purification by flash chromatography on $\mathrm{SiO}_{2}(7.5: 1$ hexanes/EtOAc) yielded $2.57 \mathrm{~g}(89 \%)$ of the title compound as a yellow oil: ${ }^{1} \mathrm{H} \mathrm{NMR}\left(300 \mathrm{MHz}, \mathrm{CDCl}_{3}\right): \delta$ 7.46-7.22 (m, 5H), $6.58(\mathrm{~d}, J=16.0 \mathrm{~Hz}, 1 \mathrm{H}), 6.23(\mathrm{dd}, J=15.9,6.8 \mathrm{~Hz}, 1 \mathrm{H}), 4.29(\mathrm{q}, J=6.5 \mathrm{~Hz}, 1 \mathrm{H}), 1.69-1.50$ (m, 2H), 1.49-1.30 (m, 4H), $0.93(\mathrm{t}, J=6.7 \mathrm{~Hz}, 3 \mathrm{H})$.

\footnotetext{
${ }^{1}$ Onderdelinden, A. L.; van der Ent, A. Inorg. Chim. Acta 1972, 6, 420.

${ }^{2}$ Trost, B. M.; Kulawiec, R. J. J. Am. Chem. Soc. 1993, 115, 2027.
} 
1-Phenyl-but-2-en-1-ol (7b): ${ }^{3}$ To $4.74 \mathrm{~g}(3.18 \mathrm{~mL}, 30.2 \mathrm{mmol})$ of bromobenzene in $200 \mathrm{~mL}$ of $\mathrm{Ph} \mathrm{Me}_{2} \mathrm{O}$ at $-78^{\circ} \mathrm{C}$ was added $35.5 \mathrm{~mL}(60.4 \mathrm{mmol})$ of tert-butyllithium in pentane (1.7 M). Following $1 \mathrm{~h}, 2.51 \mathrm{~mL}(2.12 \mathrm{~g}, 30.2 \mathrm{mmol})$ of crotonaldehyde was added slowly via syringe, and the mixture was stirred an additional $20 \mathrm{~min}$. The reaction was quenched carefully with water and slowly raised to ambient temperature. The aqueous layer was extracted with $\mathrm{Et}_{2} \mathrm{O}(3 \mathrm{x})$, the combined organic layers were dried over $\mathrm{Na}_{2} \mathrm{SO}_{4}$, filtered and the crude product mixture was concentrated in vacuo. The crude product was used as isolated: ${ }^{1} \mathrm{H}$ NMR $\left(300 \mathrm{MHz}, \mathrm{CDCl}_{3}\right): \delta 7.40-7.27(\mathrm{~m}, 5 \mathrm{H}), 5.85-5.67(\mathrm{~m}, 2 \mathrm{H}), 5.18(\mathrm{br} . \mathrm{dd}, J=6.4,3.2 \mathrm{~Hz}, 1 \mathrm{H})$, $1.85(\mathrm{~d}, J=3.5 \mathrm{~Hz}, 1 \mathrm{H}), 1.73(\mathrm{~d}, J=5.5 \mathrm{~Hz}, 3 \mathrm{H})$.

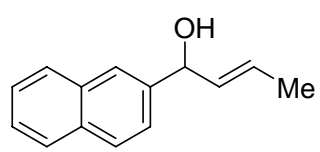

1-Naphthalen-2-yl-but-2-en-1-ol (7c): ${ }^{4}$ To $1.2 \mathrm{~g}$ (49 mol) of mechanically activated $\operatorname{Mg}(0)$ was added $15 \mathrm{~mL}$ of THF and $\mathrm{I}_{2}$ (cat., in $0.5 \mathrm{~mL}$ of THF). Initiation was afforded by brief warming with a heatgun (brown $\rightarrow$ clear/white color shift), after which $0.010 \mathrm{~kg}$ (48 mmol) of 2-bromonapthalene in $10 \mathrm{~mL}$ of THF was carefully added over $30 \mathrm{~min}$ to maintain a gentle reflux. The mixture was refluxed $15 \mathrm{~min}$ longer with a heatgun, then stirred at ambient temperature for $1 \mathrm{~h}$. In a separate flask, $4.8 \mathrm{~mL}(4.1 \mathrm{~g})$ of crotonaldehyde was dissolved in $19 \mathrm{~mL}$ of THF and the temperature reduced to $-78^{\circ} \mathrm{C}$. The active Grignard reagent was added via syringe over 15 min and after $1 \mathrm{~h}$, the reaction was quenched carefully with sat. aq. $\mathrm{NH}_{4} \mathrm{Cl}$ and raised to ambient temperature. The aqueous layer was extracted with $\mathrm{Et}_{2} \mathrm{O}(3 \mathrm{x})$, the combined organic layers were dried over $\mathrm{Na}_{2} \mathrm{SO}_{4}$, filtered and the crude product mixture was concentrated in vacuo. The product was purified by flash chromatography on $\mathrm{SiO}_{2}(6: 1$ hexanes/EtOAc) to afford $7.5 \mathrm{~g}(79 \%)$ of the product as a highly viscous, yellow oil: ${ }^{1} \mathrm{H} \mathrm{NMR}(300 \mathrm{MHz}$, $\left.\mathrm{CDCl}_{3}\right): \delta 7.86-7.82(\mathrm{~m}, 4 \mathrm{H}), 7.51-7.45(\mathrm{~m}, 3 \mathrm{H}), 5.90-5.73(\mathrm{~m}, 2 \mathrm{H}), 5.35(\mathrm{~d}, J=5.1 \mathrm{~Hz}, 1 \mathrm{H}), 2.00(\mathrm{~s}, 1 \mathrm{H}), 1.76$ $(\mathrm{d}, J=5.6 \mathrm{~Hz}, 3 \mathrm{H})$.

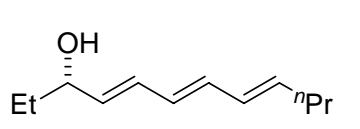

(S,4E,6E,8E)-Dodeca-4,6,8-trien-3-ol (7d): Both the trienal aldehyde and MIB ligand were prepared according to literature procedures. ${ }^{5,6}$ To a solution of $41 \mathrm{mg}(0.17 \mathrm{mmol})$ of MIB in $3.3 \mathrm{~mL}$ toluene was added $6.6 \mathrm{~mL}(6.6 \mathrm{mmol})$ of $\mathrm{Et}_{2} \mathrm{Zn}$ in hexanes $(1.0 \mathrm{M})$ at ambient temperature. Following $30 \mathrm{~min}$, the flask was immersed in an ice bath and $0.50 \mathrm{~g}(3.3 \mathrm{mmol})$ of the aldehyde was added dropwise by syringe. The reaction was stirred at $0{ }^{\circ} \mathrm{C}$ for $30 \mathrm{~min}$, then quenched carefully with sat. aq. Rochelle's salt and stirred vigorously for $30 \mathrm{~min}$ while warming to ambient temperature. The aqueous layer was extracted with EtOAc (3x), the combined organic layers were dried over $\mathrm{Na}_{2} \mathrm{SO}_{4}$, filtered, and the crude product mixture was concentrated in vacuo. Purification by flash chromatography on $\mathrm{SiO}_{2}(6: 1$ hexanes/EtOAc) yielded $0.43 \mathrm{~g}(73 \%)$ of the title compound as a clear oil. Separation of the enantiomers by chiral HPLC (Daicel Chirace ${ }^{\mathrm{TM}}$ OD-H column, flow rate $1.0 \mathrm{ml} / \mathrm{min}, 2.0 \% \mathrm{i}$-PrOH, $98.0 \%$ hexanes) provided the enantiomeric ratio: $(S)-7 \mathbf{d}(\mathrm{Tr}=12.1):(R)-7 \mathbf{d}(\mathrm{Tr}=13.3) 94.5: 5.5(89 \%$ ee $):[\alpha]_{\mathrm{D}}=+24.0^{\circ}\left(\mathrm{c} 1.24, \mathrm{CHCl}_{3}\right) ; \mathrm{IR}$ (thin film) 3354, 3015, 2961, 1636, 1436, $995 \mathrm{~cm}^{-1} ;{ }^{1} \mathrm{H}$ NMR $\left(500 \mathrm{MHz}, \mathrm{CDCl}_{3}\right): \delta 6.26-6.18(\mathrm{~m}, 2 \mathrm{H}), 6.11(\mathrm{dd}, J$ $=15.0,10.4 \mathrm{~Hz}, 1 \mathrm{H}), 6.07(\mathrm{dd}, J=15.0,10.6 \mathrm{~Hz}, 1 \mathrm{H}), 5.73(\mathrm{dt}, J=14.5,7.1 \mathrm{~Hz}, 1 \mathrm{H}), 5.66(\mathrm{dd}, J=15.2,6.9 \mathrm{~Hz}$, $1 \mathrm{H}), 4.11-4.06(\mathrm{~m}, 1 \mathrm{H}), 2.09(\mathrm{q}, J=7.2 \mathrm{~Hz}, 2 \mathrm{H}), 1.65-1.51(\mathrm{~m}, 2 \mathrm{H}), 1.46-1.39(\mathrm{~m}, 3 \mathrm{H}), 0.93(\mathrm{t}, J=7.1 \mathrm{~Hz}, 3 \mathrm{H})$, $0.91(\mathrm{t}, J=7.1 \mathrm{~Hz}, 3 \mathrm{H}) ;{ }^{13} \mathrm{C} \mathrm{NMR}\left(75 \mathrm{MHz}, \mathrm{CDCl}_{3}\right): \delta 135.6,135.0,133.4,130.9,130.3,129.6,74.0,34.8,30.1$, 22.4, 13.6, 9.6; MS (EI) m/z $180\left(\mathrm{M}^{+}\right), 162,147,133,119,105,91$; HRMS (EI) $\mathrm{m} / \mathrm{z}$ calculated for $\mathrm{C}_{12} \mathrm{H}_{20} \mathrm{O}_{1}\left(\mathrm{M}^{+}\right)$: 180.1514 , found 180.1506 .

General Procedure A for Preparing Propargylic Ethers 5a-d: To $0.080 \mathrm{~g}$ (2.0 mmol) of sodium hydride (60\% dispersion in mineral oil, pre-washed $3 \mathrm{x}$ with pentane) was added $1.4 \mathrm{~mL}$ of THF. The solution was cooled to 0 ${ }^{\circ} \mathrm{C}$, and the allylic alcohol $(1.0 \mathrm{mmol})$ was added via syringe or Pasteur pipette. The reaction was stirred at $0{ }^{\circ} \mathrm{C}$ for $\sim 15 \mathrm{~min}$, then warmed slowly to ambient temperature. At this time, a condenser was attached to the reaction vessel and the reaction mixture was heated to reflux for $30 \mathrm{~min}$, whereupon $0.30 \mathrm{~g}(2.0 \mathrm{mmol})$ of propargyl

\footnotetext{
${ }^{3}$ Pocker, Y.; Hill, M. J. J. Am. Chem. Soc. 1969, 91, 3243.

${ }^{4}$ Dishington, A. P.; Douthwaite, R. E.; Mortlock, A.; Muccioli, A. B.; Simpkins, N. S. J. Chem. Soc., Perkin Trans. 1, 1997, 323.

${ }^{5}$ Ishida, A.; Mukaiyama, T. Bull. Chem. Soc. Japan 1977, 50, 1161.

${ }^{6}$ Nugent, W. A. Chem. Commun. 1999, 1369.
} 
bromide in toluene $(80 \% / \mathrm{wt})$ was added carefully through the condenser. Following $1 \mathrm{~h}$ at reflux, the solution was cooled to ambient temperature and quenched carefully with $\mathrm{H}_{2} \mathrm{O}$. The aqueous layer was extracted with $\mathrm{Et}_{2} \mathrm{O}$ $(3 \mathrm{x})$, the combined organic layers were dried over $\mathrm{MgSO}_{4}$, and the solvent was filtered and removed in vacuo. The product was purified as indicated.

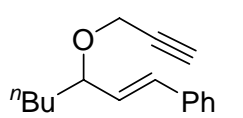

(3-Prop-2-ynyloxy-hept-1-enyl)benzene (5a): The general procedure $\mathbf{A}$ was followed employing $1.14 \mathrm{~g}(6.00 \mathrm{mmol})$ of allylic alcohol 7a. Purification by flash chromatography on $\mathrm{SiO}_{2}(40: 1$ hexanes/EtOAc) afforded $1.03 \mathrm{~g}(75 \%)$ of the product as a red-orange oil. Further purification was accomplished by distillation at low pressure $\left(\sim 100^{\circ} \mathrm{C}\right)$ : IR (thin film) $3301,3027,2956,2116$, 1494, 1071, 969, 750, $693 \mathrm{~cm}^{-1} ;{ }^{1} \mathrm{H}$ NMR $\left(300 \mathrm{MHz}, \mathrm{CDCl}_{3}\right): \delta$ 7.43-7.24 (m, 5H), $6.58(\mathrm{~d}, J=15.9 \mathrm{~Hz}, 1 \mathrm{H})$, $6.03(\mathrm{dd}, J=15.9,8.3 \mathrm{~Hz}, 1 \mathrm{H}), 4.23(\mathrm{dd}, J=15.6,2.1 \mathrm{~Hz}, 1 \mathrm{H}), 4.08(\mathrm{dd}, J=15.5,2.1 \mathrm{~Hz}, 1 \mathrm{H}), 4.05(\mathrm{q}, J=6.7$ $\mathrm{Hz}, 1 \mathrm{H}), 2.42(\mathrm{t}, J=2.2 \mathrm{~Hz}, 1 \mathrm{H}), 1.78-1.69(\mathrm{~m}, 1 \mathrm{H}), 1.65-1.53(\mathrm{~m}, 1 \mathrm{H}), 1.49-1.32(\mathrm{~m}, 4 \mathrm{H}), 0.91(\mathrm{t}, J=6.7 \mathrm{~Hz}$, $3 \mathrm{H}) ;{ }^{13} \mathrm{C}$ NMR $\left(75 \mathrm{MHz}, \mathrm{CDCl}_{3}\right.$ ): $\delta 136.3,133.0,129.3,128.5,127.7,126.4,80.2,79.6,73.8,55.0,35.2,27.4$, 22.5, 13.9; MS (EI) m/z $228\left(\mathrm{M}^{+}\right), 198,189,171,131,85,57$; HRMS (EI) $m / z$ calculated for $\mathrm{C}_{16} \mathrm{H}_{20} \mathrm{O}: 228.1514$, found 228.1508 .

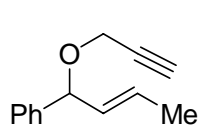

(1-Prop-2-ynyloxy-but-2-enyl)benzene (5b): The general procedure A was followed employing $889 \mathrm{mg}(6.00 \mathrm{mmol})$ of allylic alcohol $\mathbf{7 b}$. The product was purified by flash chromatography on $\mathrm{SiO}_{2}(40: 1$ hexanes/EtOAc) to afford $939 \mathrm{mg}(84 \%)$ of the product as a red-orange oil: IR (thin film) $3295,3029,2916,2116,1493,1451,1062,968,755,700 \mathrm{~cm}^{-1} ;{ }^{1} \mathrm{H}$ NMR $\left(300 \mathrm{MHz}, \mathrm{CDCl}_{3}\right)$ : $\delta$ 7.39-7.26 (m, 5H), $5.78(\mathrm{dq}, J=15.3,6.2 \mathrm{~Hz}, 1 \mathrm{H}), 5.60(\mathrm{ddq}, J=15.3,7.2,1.3 \mathrm{~Hz}, 1 \mathrm{H}), 4.98(\mathrm{~d}, J=7.4 \mathrm{~Hz}$ $1 \mathrm{H}), 4.17$ (dd, $J=15.7,2.4 \mathrm{~Hz}, 1 \mathrm{H}), 4.09$ (dd, $J=15.7,2.4 \mathrm{~Hz}, 1 \mathrm{H}), 2.42$ (t, $J=2.4 \mathrm{~Hz}, 1 \mathrm{H}), 1.74(\mathrm{dd}, J=6.4$, $1.4 \mathrm{~Hz}, 3 \mathrm{H}) ;{ }^{13} \mathrm{C}$ NMR $\left(75 \mathrm{MHz}, \mathrm{CDCl}_{3}\right): \delta 140.7,131.1,129.1,128.3,127.5,126.8,80.8,79.9,74.1,54.7,17.1$; MS (EI) $\mathrm{m} / \mathrm{z} 186\left(\mathrm{M}^{+}\right), 171,147,131,105,91,77,69$; HRMS (EI) $\mathrm{m} / \mathrm{z}$ calculated for $\mathrm{C}_{13} \mathrm{H}_{14} \mathrm{O}: 186.1045$, found 186.1039 .

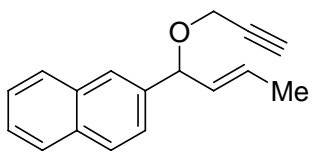

2-(1-Prop-2-ynyloxy-but-2-enyl)naphthalene (5c): The general procedure $\mathbf{A}$ was followed employing $1.42 \mathrm{~g}(7.16 \mathrm{mmol})$ of allylic alcohol 7c, $0.480 \mathrm{~g}(12.0 \mathrm{mmol})$ of $\mathrm{NaH}$, and $1.79 \mathrm{~g}(12.0 \mathrm{mmol})$ of propargyl bromide. Purification by flash chromatography on $\mathrm{SiO}_{2}(50: 1 \rightarrow 25: 1$ hexanes/EtOAc) afforded $1.23 \mathrm{~g}(73 \%)$ of the product as a viscous, redorange oil. Further purification was accomplished by distillation at low pressure $\left(\sim 120^{\circ} \mathrm{C}\right)$ : IR (thin film) 3293 , $3056,2854,2116,1508,1440,1062,967,750 \mathrm{~cm}^{-1} ;{ }^{1} \mathrm{H}$ NMR $\left(300 \mathrm{MHz}, \mathrm{CDCl}_{3}\right): \delta 7.86-7.82(\mathrm{~m}, 4 \mathrm{H}), 7.50-7.45$ $(\mathrm{m}, 3 \mathrm{H}), 5.83(\mathrm{dq}, J=15.2,6.2 \mathrm{~Hz}, 1 \mathrm{H}), 5.68(\mathrm{ddq}, J=15.4,7.2,1.4 \mathrm{~Hz}, 1 \mathrm{H}), 5.15(\mathrm{~d}, J=7.2 \mathrm{~Hz}, 1 \mathrm{H}), 4.22(\mathrm{dd}$, $J=15.6,2.3 \mathrm{~Hz}, 1 \mathrm{H}), 4.12(\mathrm{dd}, J=15.8,2.3 \mathrm{~Hz}, 1 \mathrm{H}), 2.45(\mathrm{t}, J=2.4 \mathrm{~Hz}, 1 \mathrm{H}), 1.75(\mathrm{~d}, J=6.4 \mathrm{~Hz}, 3 \mathrm{H}) ;{ }^{13} \mathrm{C}$ NMR $\left(75 \mathrm{MHz}, \mathrm{CDCl}_{3}\right): \delta 138.0,133.2,133.0,131.0,129.5,128.2,127.9,127.6,126.0,125.8,125.7,124.8,81.0,79.9$, 74.3, 55.0, 17.7; MS (EI) m/z $236\left(\mathrm{M}^{+}\right), 221,197,179,155,141,127$; HRMS (EI) $\mathrm{m} / \mathrm{z}$ calculated for $\mathrm{C}_{17} \mathrm{H}_{16} \mathrm{O}$ : 236.1201, found 236.1201 .

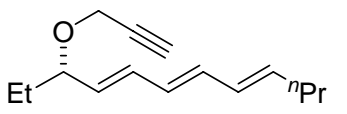

(S,4E,6E,8E)-3-(Prop-2-ynyloxy)dodeca-4,6,8-triene (5d): The general procedure A was followed employing $2.1 \mathrm{~g}(12 \mathrm{mmol})$ of trienyl alcohol $7 \mathbf{d}$ with $1.5 \mathrm{~h}$ heating time. Purification by flash chromatography on $\mathrm{SiO}_{2}(50: 1$ hexanes/EtOAc) afforded $2.2 \mathrm{~g}$ $(83 \%)$ of the product as a yellow oil: $[\alpha]_{\mathrm{D}}=-141\left(\mathrm{c} 1.32, \mathrm{CHCl}_{3}\right)$; IR (thin film) 3307, 3016, 2962, 2116, 1636, 1463, 1072, 997, $663 \mathrm{~cm}^{-1}$; ${ }^{1} \mathrm{H}$ NMR $\left(300 \mathrm{MHz}, \mathrm{CDCl}_{3}\right): \delta 6.27-6.04(\mathrm{~m}, 4 \mathrm{H}), 5.75(\mathrm{dt}, J=14.6,7.0 \mathrm{~Hz}, 1 \mathrm{H})$, $5.44(\mathrm{dd}, J=15.4,8.4 \mathrm{~Hz}, 1 \mathrm{H}), 4.17(\mathrm{dd}, J=15.6,2.3 \mathrm{~Hz}, 1 \mathrm{H}), 4.01(\mathrm{dd}, J=15.7,2.3 \mathrm{~Hz}, 1 \mathrm{H}), 3.84(\mathrm{dt}, J=8.3$, $6.5 \mathrm{~Hz}, 1 \mathrm{H}), 2.39$ (t, $J=2.3 \mathrm{~Hz}, 1 \mathrm{H}), 2.09$ (q, $J=7.2 \mathrm{~Hz}, 2 \mathrm{H}), 1.72-1.61(\mathrm{~m}, 1 \mathrm{H}), 1.60-1.48(\mathrm{~m}, 1 \mathrm{H}), 1.48-1.37$ $(\mathrm{m}, 2 \mathrm{H}), 0.91(\mathrm{t}, J=7.3 \mathrm{~Hz}, 3 \mathrm{H}), 0.91(\mathrm{t}, J=7.5 \mathrm{~Hz}, 3 \mathrm{H}) ;{ }^{13} \mathrm{C}$ NMR $\left(75 \mathrm{MHz}, \mathrm{CDCl}_{3}\right): \delta 136.0,133.8(2 \mathrm{C})$, 131.8, 130.2, 129.4, 80.9. 80.4, 73.6, 55.1, 34.9, 28.4, 22.4, 13.6, 9.7; MS (EI) m/z $218\left(\mathrm{M}^{+}\right), 189,162,133,119$, 107, 91, 79; HRMS (EI) $\mathrm{m} / \mathrm{z}$ calculated for $\mathrm{C}_{15} \mathrm{H}_{22} \mathrm{O}_{1}\left(\mathrm{M}^{+}\right): 218.1671$, found 218.1665. 
General Procedure B for Preparing Vinyl Boronic Esters 6a-d: ${ }^{7}$ The alkyne (1.0 equiv) was added to a suspension of $\mathrm{Cp}_{2} \mathrm{Zr}(\mathrm{H}) \mathrm{Cl}^{8}\left(0.05\right.$ equiv) in $\mathrm{CH}_{2} \mathrm{Cl}_{2}(3.0 \mathrm{M})$ at $0{ }^{\circ} \mathrm{C}$ in a microwave reaction vessel. Pinacolborane (1.1 equiv) was added and the resulting suspension was warmed directly to ambient temperature, then heated at $100{ }^{\circ} \mathrm{C}$ in a microwave reactor for $45 \mathrm{~min}$. The solvent was removed in vacuo and the residue purified by flash chromatography on Iatrobeads 6RS-8060 silica gel.

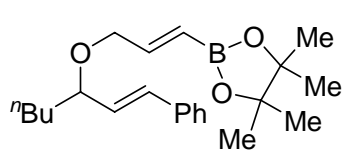

2-\{(1E)-3-[(E)-1-Phenylhept-1-en-3-yloxy]prop-1-enyl $\}-4,4,5,5-$ tetramethyl-1,3,2-dioxaborolane (6a): General Procedure $\mathbf{B}$ was followed employing $1.00 \mathrm{~g}$ of 1-[(E)-3-(prop-2-ynyloxy)hept-1-enyl]benzene $(4.38 \mathrm{mmol})$. Purification by flash chromatography ( $5 \%$ EtOAc/hexanes) gave $1.18 \mathrm{~g} \mathrm{(75 \% )} \mathrm{of} \mathrm{the} \mathrm{title} \mathrm{compound} \mathrm{as}$ a colorless oil: ${ }^{1} \mathrm{H}$ NMR $\left(300 \mathrm{MHz}, \mathrm{CDCl}_{3}\right): \delta 7.42-7.20(\mathrm{~m}, 5 \mathrm{H}), 6.66(\mathrm{dt}, J=18,4.6 \mathrm{~Hz}, 1 \mathrm{H}), 6.49(\mathrm{~d}, J=16$ $\mathrm{Hz}, 1 \mathrm{H}), 6.05$ (dd, $J=16,8.0 \mathrm{~Hz}, 1 \mathrm{H}), 5.73$ (dt, $J=18,1.8 \mathrm{~Hz}, 1 \mathrm{H}), 4.15$ (ddd, $J=15,4.4,1.9 \mathrm{~Hz}, 1 \mathrm{H}), 3.96$ (ddd, $J=15,4.8,1.8 \mathrm{~Hz}, 1 \mathrm{H}), 3.85(\mathrm{dt}, J=6.5,7.3 \mathrm{~Hz}, 1 \mathrm{H}), 1.78-1.50(\mathrm{~m}, 2 \mathrm{H}), 1.27(\mathrm{~s}, 12 \mathrm{H}), 1.40-1.24(\mathrm{~m}, 4 \mathrm{H})$, $0.89(\mathrm{~m}, 3 \mathrm{H}) ;{ }^{13} \mathrm{C}$ NMR $\left(75 \mathrm{MHz}, \mathrm{CDCl}_{3}\right): \delta 149.8,136.5,132.0,130.5,128.4,127.5,126.3,118.6$ (br), 83.0, 80.4, 69.5, 35.5, 27.4, 24.6, 22.6, 14.0; MS (EI) $\mathrm{m} / \mathrm{z} 356\left(\mathrm{M}^{+}\right), 341,326,299,270,257,199,173,167,155,143$, $131,117,105,91,85,77,67,57$; HRMS $m / z$ calculated for $\mathrm{C}_{22} \mathrm{H}_{33}{ }^{11} \mathrm{BO}_{3}: 356.2523$, found 356.2523 .

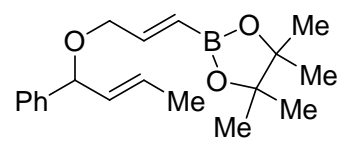

2-\{(1E)-3-[(E)-1-Phenylbut-2-enyloxy]prop-1-enyl\}-4,4,5,5-tetramethyl-1,3,2dioxaborolane (6b): General Procedure B was followed employing $1.14 \mathrm{~g}$ of 1-[(E)-1(prop-2-ynyloxy)but-2-enyl]benzene $\quad(6.14 \mathrm{mmol})$. Purification by flash chromatography $(5 \%$ EtOAc/hexanes) gave $1.60 \mathrm{~g}(83 \%)$ of the title compound as a colorless oil: ${ }^{1} \mathrm{H}$ NMR $\left(300 \mathrm{MHz}, \mathrm{CDCl}_{3}\right): \delta 7.40-7.20(\mathrm{~m}, 5 \mathrm{H}), 6.67(\mathrm{dt}, J=18,4.5 \mathrm{~Hz}, 1 \mathrm{H}), 5.76(\mathrm{dt}, J=16,1.8$ $\mathrm{Hz}, 1 \mathrm{H}), 5.70(\mathrm{ddq}, J=15,5.9,0.6 \mathrm{~Hz}, 1 \mathrm{H}), 5.57(\mathrm{ddq}, J=15,7.2 .1 .2 \mathrm{~Hz}, 1 \mathrm{H}), 4.74(\mathrm{~d}, J=7.0 \mathrm{~Hz}, 1 \mathrm{H}), 4.07$ (ddd, $J=15,4.5,1.8 \mathrm{~Hz}, 1 \mathrm{H}), 4.00(\mathrm{ddd}, J=15,4.5,1.8 \mathrm{~Hz}, 1 \mathrm{H}), 1.70(\mathrm{dd}, J=6.3,1.3 \mathrm{~Hz}, 3 \mathrm{H}), 1.27(\mathrm{~s}, 12 \mathrm{H})$; ${ }^{13} \mathrm{C}$ NMR $\left(75 \mathrm{MHz}, \mathrm{CDCl}_{3}\right): \delta 149.4,141.5,132.0,128.2,128.0,127.2,126.5,118.9$ (br), 83.0, 81.8, 69.3, 24.6, 17.6; MS (EI) m/z $314\left(\mathrm{M}^{+}\right), 299,284,271,256,230,214,208,199,169,147,131,119,91,85,69,59$; HRMS $\mathrm{m} / \mathrm{z}$ calculated for $\mathrm{C}_{19} \mathrm{H}_{27}{ }^{11} \mathrm{BO}_{3}: 314.2053$, found 314.2060 .

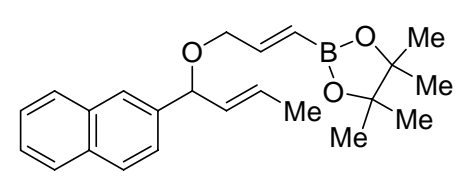

2-\{(1E)-3-[(E)-1-(Naphthalen-2-yl)but-2-enyloxy]prop-1-enyl $\}-4,4,5,5-$ tetramethyl-1,3,2-dioxaborolane (6c): General Procedure B was followed employing $1.00 \mathrm{~g}$ of 2-[(E)-1-(prop-2-ynyloxy)but-2-enyl]naphthalene (4.23 mmol). Purification by flash chromatography ( $8 \%$ EtOAc/hexanes) gave $1.19 \mathrm{~g}$ (77\%) of the title compound as a colorless oil: ${ }^{1} \mathrm{H}$ NMR $\left(300 \mathrm{MHz}, \mathrm{CDCl}_{3}\right): \delta 7.81(\mathrm{~m}, 4 \mathrm{H}), 7.46(\mathrm{~m}, 3 \mathrm{H}), 6.70$ $(\mathrm{dt}, J=18,4.5 \mathrm{~Hz}, 1 \mathrm{H}), 5.81-5.61(\mathrm{~m}, 3 \mathrm{H}), 4.92(\mathrm{~d}, J=6.6 \mathrm{~Hz}, 1 \mathrm{H}), 4.11(\mathrm{ddd}, J=15,4.5,1.8 \mathrm{~Hz}, 1 \mathrm{H}), 4.05$ $(\mathrm{ddd}, J=15,4.5,1.9 \mathrm{~Hz}, 1 \mathrm{H}), 1.72(\mathrm{~d}, J=5.8 \mathrm{~Hz}, 3 \mathrm{H}), 1.27(\mathrm{~s}, 12 \mathrm{H}) ;{ }^{13} \mathrm{C} \mathrm{NMR}\left(75 \mathrm{MHz}, \mathrm{CDCl}_{3}\right): \delta 149.4$, $138.9,133.1,132.8,131.8,128.2,128.0,127.8,127.5,125.8,125.6,125.2,124.7,118.8$ (br), 83.0, 81.9, 69.3, 24.6, 17.6; MS (EI) m/z $364\left(\mathrm{M}^{+}\right), 349,280,197,181,169,155,141,127,115,101,85,69,59 ; \mathrm{HRMS} \mathrm{m} / \mathrm{z}$ calculated for $\mathrm{C}_{23} \mathrm{H}_{29}{ }^{11} \mathrm{BO}_{3}: 364.2210$, found 364.2228 .

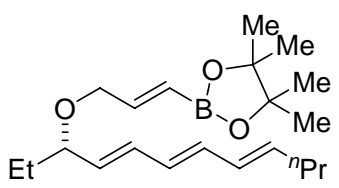

2-\{(1E)-3-[(S,4E,6E,8E)-Dodeca-4,6,8-trien-3-yloxy]prop-1-enyl\}-4,4,5,5tetramethyl-1,3,2-dioxaborolane (6d): General Procedure B was followed employing $0.88 \mathrm{~g}$ of $(S, 4 E, 6 E, 8 E)$-3-(prop-2-ynyloxy)dodeca-4,6,8-triene $(4.0 \mathrm{mmol})$, heating at 80 ${ }^{\circ} \mathrm{C}$ for $45 \mathrm{~min}$. Purification by flash chromatography (22:1 hexanes/EtOAc) gave $0.75 \mathrm{~g}$ $(55 \%)$ of the title compound as a colorless oil: $[\alpha]_{\mathrm{D}}=-14.2\left(\mathrm{c} 1.22, \mathrm{CHCl}_{3}\right)$; IR (thin film) 2976, 1644, 1463, 1354, 1146, 996, 850, $628 \mathrm{~cm}^{-1} ;{ }^{1} \mathrm{H}$ NMR $\left(500 \mathrm{MHz}, \mathrm{CDCl}_{3}\right): \delta 6.64(\mathrm{dt}, J=18.1,4.5$ $\mathrm{Hz}, 1 \mathrm{H}), 6.24-6.04(\mathrm{~m}, 4 \mathrm{H}), 5.72(\mathrm{dt}, J=14.9,7.1 \mathrm{~Hz}, 1 \mathrm{H}), 5.71(\mathrm{~d}, J=18.0 \mathrm{~Hz}, 1 \mathrm{H}), 5.48(\mathrm{dd}, J=14.4,8.1 \mathrm{~Hz}$, $1 \mathrm{H}), 4.10(\mathrm{ddd}, J=14.7,4.2,1.7 \mathrm{~Hz}, 1 \mathrm{H}), 3.91(\mathrm{ddd}, J=14.7,4.7,1.6 \mathrm{~Hz}, 1 \mathrm{H}), 3.66(\mathrm{dt}, J=7.8,6.5 \mathrm{~Hz}, 1 \mathrm{H})$, $2.08(\mathrm{q}, J=7.1,2 \mathrm{H}), 1.70-1.62(\mathrm{~m}, 1 \mathrm{H}), 1.56-1.49(\mathrm{~m}, 1 \mathrm{H}), 1.46-1.38(\mathrm{~m}, 2 \mathrm{H}), 1.27(\mathrm{~s}, 12 \mathrm{H}), 0.91(\mathrm{t}, J=7.5 \mathrm{~Hz}$, $3 \mathrm{H}), 0.89(\mathrm{t}, J=7.6 \mathrm{~Hz}, 3 \mathrm{H}) ;{ }^{13} \mathrm{C} \mathrm{NMR}\left(75 \mathrm{MHz}, \mathrm{CDCl}_{3}\right): \delta 149.9,135.6,133.3,133.2,132.8,130.3,129.6$,

\footnotetext{
${ }^{7}$ A modification of the procedure described by: Pereira, S.; Srebnik, M. Organometallics 1995, 14, $3127-3128$.

${ }^{8}$ Buchwald, S.; LaMaire, S.; Nielsen, R.; Watson, B.; King, S. Org. Synth., Coll. Vol. IX 1994, 162.
} 
118.6 (br), 83.1, 81.5, 69.6, 34.9, 28.6, 24.8, 22.4, 13.6, 9.7; MS (EI) $\mathrm{m} / \mathrm{z} 346\left(\mathrm{M}^{+}\right), 317,288,248,187,179,163$, 107; HRMS (EI) $\mathrm{m} / \mathrm{z}$ calculated for $\mathrm{C}_{21} \mathrm{H}_{35} \mathrm{BO}_{3}\left(\mathrm{M}^{+}\right)$: 346.2679, found 346.2679.

General Procedure $\mathbf{C}$ for Preparing $\boldsymbol{\beta}$-Boronic Aldehydes 8 a-d: Note that distillation of propargylic ethers 5a-c benefits the reproducibility of iridium catalyzed isomerizations. A solution of $\left[\left({ }^{c} \mathrm{C}_{8} \mathrm{H}_{14}\right)_{2} \mathrm{IrCl}\right]_{2}(1.0 \mathrm{~mol} \%$, 0.02 equiv Ir) and $\mathrm{PCy}_{3}\left(6.0 \mathrm{~mol} \%, 0.06\right.$ equiv) in anhydrous $\mathrm{CH}_{2} \mathrm{Cl}_{2}$ or 1,2-dichloroethane (1,2-DCE) was added to a solution of $\mathrm{NaBPh}_{4}\left(2.0 \mathrm{~mol} \%, 0.02\right.$ equiv) in and equal volume of $\mathrm{CH}_{2} \mathrm{Cl}_{2} /$ acetone $(25: 1)$ or 1,2DCE/acetone (25:1) (0.67M final concentration in substrate 6) and the resulting yellow solution stirred for 5 min at ambient temperature. Allyl borolane 6 (1.0 equiv) was added and the reaction stirred for 90 min at ambient temperature whereupon $\mathrm{PPh}_{3}\left(6.0 \mathrm{~mol} \%, 0.06\right.$ equiv) was added and the resulting solution heated at $\left(40\right.$ or $\left.80{ }^{\circ} \mathrm{C}\right)$ for the indicated time. The solvent was removed in vacuo and the residue purified by flash chromatography on Iatrobeads 6RS-8060 silica gel. Diastereomeric ratios were determined by integration of the specified resonances from $300 \mathrm{MHz}{ }^{1} \mathrm{H}-\mathrm{NMR}$. Satisfactory mass spectral data could not be obtained for compounds 8a-d; copies of ${ }^{1} \mathrm{H}$ and ${ }^{13} \mathrm{C}$ spectra are provided and mass spectral data is provided for all compounds derived from $\mathbf{8}$.

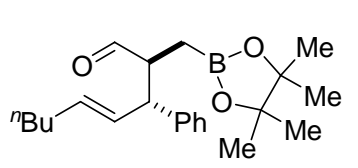

$R^{*}$-(E,2R,3S)-2-[(4,4,5,5-Tetramethyl-1,3,2-dioxaborolan-2-yl)methyl]-3-phenylnon4-enal (8a): General Procedure $\mathbf{C}\left(1,2-\mathrm{DCE}, 80^{\circ} \mathrm{C}\right)$ was followed employing $1.15 \mathrm{~g}$ of 2-((1E)-3-((E)-1-phenylhept-1-en-3-yloxy)prop-1-enyl)-4,4,5,5-tetramethyl-1,3,2dioxaborolane $(3.23 \mathrm{mmol})$ and a reaction time of 2.5 hours. Purification by flash chromatography (6\% EtOAc/hexanes) on Iatrobeads gave $0.772 \mathrm{~g}(67 \%)$ of the title compound as a colorless oil $\left(\mathrm{C} \underline{\mathbf{H}} \mathrm{O}\right.$, anti:syn = 92:8): ${ }^{1} \mathrm{H} \mathrm{NMR}\left(300 \mathrm{MHz}, \mathrm{CDCl}_{3}\right): \delta 9.77(\mathrm{~d}, J=1.8 \mathrm{~Hz}, 1 \mathrm{H}), 7.35-7.25(\mathrm{~m}, 2 \mathrm{H}), 7.25-7.15$ $(\mathrm{m}, 3 \mathrm{H}), 5.67(\mathrm{ddt}, J=15,8.7,1.2 \mathrm{~Hz}, 1 \mathrm{H}), 5.53(\mathrm{dt}, J=15,6.6 \mathrm{~Hz}, 1 \mathrm{H}), 3.53(\mathrm{t}, J=8.6 \mathrm{~Hz}, 1 \mathrm{H}), 2.94(\mathrm{~m}, 1 \mathrm{H})$, $2.00(\mathrm{~m}, 2 \mathrm{H}), 1.21(\mathrm{~s}, 6 \mathrm{H}), 1.35-1.19(\mathrm{~m}, 4 \mathrm{H}), 1.18(\mathrm{~s}, 6 \mathrm{H}), 0.87(\mathrm{~m}, 3 \mathrm{H}), 0.80(\mathrm{dd}, J=16,9.6 \mathrm{~Hz}, 1 \mathrm{H}), 0.68(\mathrm{dd}$, $J=16,5.0 \mathrm{~Hz}, 1 \mathrm{H}) ;{ }^{13} \mathrm{C} \mathrm{NMR}\left(75 \mathrm{MHz}, \mathrm{CDCl}_{3}\right): \delta 205.1,142.1,132.9,129.9,128.4,127.8,126.4,83.0,52.6$, 51.1, 32.1, 31.3, 24.7, 24.5, 22.0, 13.8, 9.7 (br).

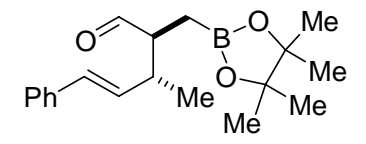

$R^{*}$-(E,2R,3S)-3-Methyl-2-[(4,4,5,5-tetramethyl-1,3,2-dioxaborolan-2-yl)methyl]-5phenylpent-4-enal (8b): General Procedure $\mathbf{C}\left(\mathrm{CH}_{2} \mathrm{Cl}_{2}, 40{ }^{\circ} \mathrm{C}\right)$ was followed employing $0.500 \mathrm{~g}$ of 2-((1E)-3-((E)-1-phenylbut-2-enyloxy)prop-1-enyl)-4,4,5,5-tetramethyl-1,3,2dioxaborolane $(1.59 \mathrm{mmol})$ and a reaction time of 4 hours. Purification by flash chromatography $(8 \% \mathrm{EtOAc} /$ hexanes $)$ on Iatrobeads gave $0.326 \mathrm{~g}(65 \%)$ of the title compound as a colorless oil (vinyl $\mathrm{C} \underline{\mathbf{H}}$, syn:anti = 92:8): ${ }^{1} \mathrm{H}$ NMR $\left(300 \mathrm{MHz}, \mathrm{CDCl}_{3}\right): \delta 9.76(\mathrm{~d}, J=0.8 \mathrm{~Hz}, 1 \mathrm{H}), 7.36-7.20(\mathrm{~m}, 5 \mathrm{H}), 6.42(\mathrm{~d}$, $J=16 \mathrm{~Hz}, 1 \mathrm{H}), 6.19(\mathrm{dd}, J=16,7.5 \mathrm{~Hz}, 1 \mathrm{H}), 2.85(\mathrm{~m}, 1 \mathrm{H}), 2.70(\mathrm{~m}, 1 \mathrm{H}), 1.24(\mathrm{~s}, 6 \mathrm{H}), 1.21(\mathrm{~s}, 6 \mathrm{H}), 1.12(\mathrm{~d}, J=$ $6.9 \mathrm{~Hz}, 1 \mathrm{H}), 1.00(\mathrm{dd}, J=16,10 \mathrm{~Hz}, 1 \mathrm{H}), 0.82(\mathrm{dd}, J=16,5.0 \mathrm{~Hz}, 1 \mathrm{H}) ;{ }^{13} \mathrm{C} \mathrm{NMR}\left(75 \mathrm{MHz}, \mathrm{CDCl}_{3}\right): \delta 204.8$, $137.1,132.7,130.1,128.4,127.1,126.0,83.1,53.2,37.8,24.7,24.5,16.4,6.7$ (br).

$R^{*}$-(E,2R,3S)-3-Methyl-2-[(4,4,5,5-tetramethyl-1,3,2-dioxaborolan-2-

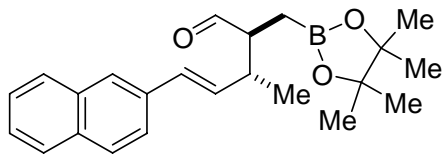
yl)methyl]-5-(naphthalen-2-yl)pent-4-enal (8c): General Procedure $\mathbf{C}\left(\mathrm{CH}_{2} \mathrm{Cl}_{2}\right.$, $\left.40{ }^{\circ} \mathrm{C}\right)$ was followed employing $0.264 \mathrm{~g}$ of 2-((1E)-3-((E)-1-(naphthalen-2-yl)but2-enyloxy)prop-1-enyl)-4,4,5,5-tetramethyl-1,3,2-dioxaborolane $(0.723 \mathrm{mmol})$ and a reaction time of 4 hours. Purification by flash chromatography $(10 \%$ EtOAc/hexanes) on Iatrobeads gave $0.159 \mathrm{~g}(61 \%)$ of the title compound as a colorless oil (Cㅡㅇ, syn:anti $=$ 91:9). Slow evaporation from pentane at $-22{ }^{\circ} \mathrm{C}$ afforded crystals which were suitable for X-Ray analysis: ${ }^{1} \mathrm{H}$ NMR (300 MHz, $\left.\mathrm{CDCl}_{3}\right): \delta 9.79(\mathrm{~d}, J=0.9 \mathrm{~Hz}, 1 \mathrm{H}), 7.80-7.75(\mathrm{~m}, 3 \mathrm{H}), 7.69(\mathrm{~s}, 1 \mathrm{H}), 7.56(\mathrm{dd}, J=8.6,1.7 \mathrm{~Hz}$, $1 \mathrm{H}), 7.48-7.39(\mathrm{~m}, 2 \mathrm{H}), 6.58(\mathrm{~d}, J=16 \mathrm{~Hz}, 1 \mathrm{H}), 6.32(\mathrm{dd}, J=16,7.5 \mathrm{~Hz}, 1 \mathrm{H}), 2.91(\mathrm{~m}, 1 \mathrm{H}), 2.76(\mathrm{~m}, 1 \mathrm{H}), 1.24$ $(\mathrm{s}, 6 \mathrm{H}), 1.21(\mathrm{~s}, 6 \mathrm{H}), 1.16(\mathrm{~d}, J=6.9 \mathrm{~Hz}, 3 \mathrm{H}), 1.04(\mathrm{dd}, J=16,10 \mathrm{~Hz}, 1 \mathrm{H}), 0.86(\mathrm{dd}, J=16,5.1 \mathrm{~Hz}, 1 \mathrm{H}) ;{ }^{13} \mathrm{C}$ NMR $\left(75 \mathrm{MHz}, \mathrm{CDCl}_{3}\right): \delta 204.7,134.5,133.5,133.1,132.7,130.2,128.0,127.7,127.5,126.1,125.7,125.5$, $123.4,83.1,53.2,37.9,24.7,24.5,16.4,6.9$ (br). 


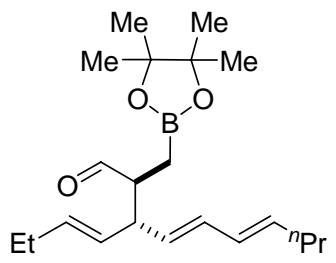

$(2 S, 3 S, 4 E, 6 E)-3-[(E)-B u t-1-e n y l]-2-[(4,4,5,5$-tetramethyl-1,3,2-dioxaborolan-2yl)methyl]deca-4,6-dienal (8d): General Procedure C $\left(1,2-\mathrm{DCE}, 80{ }^{\circ} \mathrm{C}\right)$ was followed employing $0.67 \mathrm{~g}$ of $2-\{(1 E)-3-[(S, 4 E, 6 E, 8 E)$-dodeca-4,6,8-trien-3-yloxy]prop-1-enyl $\}$ 4,4,5,5-tetramethyl-1,3,2-dioxaborolane $(1.9 \mathrm{mmol})$ and a reaction time of 5 hours. Purification by flash chromatography (18:1 hexanes:EtOAc) on Iatrobeads gave $0.39 \mathrm{~g}$ $(58 \%)$ of the title compound as an orange oil $(\mathrm{CHO},=88: 7: 5):[\alpha]_{\mathrm{D}}=+24.7(\mathrm{c} 1.47$, $\mathrm{CHCl}_{3}$ ); IR (thin film) 2963, 2725, 1724, 1461, 1371, 1146, 989, $847 \mathrm{~cm}^{-1} ;{ }^{1} \mathrm{H}$ NMR $\left(300 \mathrm{MHz}, \mathrm{C}_{6} \mathrm{D}_{6}\right): \delta 9.68(\mathrm{~d}$, $J=0.8 \mathrm{~Hz}, 1 \mathrm{H}), 6.03(\mathrm{dd}, J=14.4,10.4 \mathrm{~Hz}, 1 \mathrm{H}), 5.95(\mathrm{dd}, J=14.4,10.3 \mathrm{~Hz}, 1 \mathrm{H}), 5.51(\mathrm{dd}, J=14.3,7.0 \mathrm{~Hz}$, $1 \mathrm{H}), 5.46-5.33(\mathrm{~m}, 2 \mathrm{H}), 5.29(\mathrm{dd}, J=15.4,7.3 \mathrm{~Hz}, 1 \mathrm{H}), 3.00(\mathrm{q}, J=7.0 \mathrm{~Hz}, 1 \mathrm{H}), 2.70(\mathrm{br} \mathrm{dt}, J=9.9,5.9 \mathrm{~Hz}, 1 \mathrm{H})$, $1.97-1.80(\mathrm{~m}, 2 \mathrm{H}), 1.36-1.23(\mathrm{~m}, 1 \mathrm{H}), 1.17(\mathrm{dd}, J=16.0,10.0 \mathrm{~Hz}, 1 \mathrm{H}), 1.10(\mathrm{~s}, 6 \mathrm{H}), 1.09(\mathrm{~s}, 6 \mathrm{H}), 0.96(\mathrm{dd}, J=$ $16.0,4.8 \mathrm{~Hz}, 1 \mathrm{H}), 0.83(\mathrm{t}, J=7.4 \mathrm{~Hz}, 3 \mathrm{H}), 0.82(\mathrm{t}, J=7.3 \mathrm{~Hz}, 3 \mathrm{H}) ;{ }^{13} \mathrm{C} \mathrm{NMR}\left(75 \mathrm{MHz}, \mathrm{CDCl}_{3}\right): \delta 204.9,134.5$, 133.9, 131.9, 130.9, 130.0, 128.4, 83.2, 52.5, 47.7, 34.7, 25.6, 24.8, 24.7, 22.4, 13.7 (B- $\underline{\mathbf{C}}=$ unobserved).

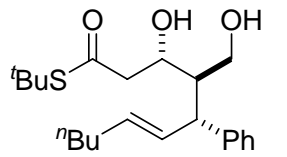

$R^{*}$-3-Hydroxy-4-hydroxymethyl-5-phenylundec-6-enethioic acid S-tert-butyl ester (11): To $0.15 \mathrm{~g}(0.42 \mathrm{mmol})$ of $\beta$-boronic aldehyde $8 \mathrm{a}\left(92: 8 \mathrm{syn}\right.$ : anti) in $4.2 \mathrm{~mL} \mathrm{CH}_{2} \mathrm{Cl}_{2}$ was added $112 \mathrm{mg}(0.547 \mathrm{mmol})$ of (1-tert-butylsulfanyl-vinyloxy)-trimethyl-silane, ${ }^{9}$ and the flask was immersed in a $-78{ }^{\circ} \mathrm{C}$ bath. To the mixture was added $0.63 \mathrm{~mL}(0.63 \mathrm{mmol})$ of dimethylaluminum chloride in hexanes $(1.0 \mathrm{M})$ dropwise and the reaction was stirred for $1 \mathrm{~h}$ at $-78^{\circ} \mathrm{C}$. The reaction was quenched with $10 \% \mathrm{w} / \mathrm{w}$ citric acid in $\mathrm{MeOH}$ and slowly raised to ambient temperature. Water was added to form a biphasic mixture, and the aqueous layer was extracted with $\mathrm{Et}_{2} \mathrm{O}(3 \mathrm{x})$. The combined organic layers were dried over $\mathrm{Na}_{2} \mathrm{SO}_{4}$, filtered, and the solvent removed. The crude borane was passed through a plug of silica (5:1 hexanes:EtOAc) and isolated in vacuo. The compound mixture was then subject to $12 \mathrm{~mL}$ of a $2: 1: 1$ $\mathrm{CH}_{2} \mathrm{Cl}_{2}$ : aq. $1 \mathrm{M} \mathrm{NaOH}: 30 \% \mathrm{HOOH}$ solution for $1.5 \mathrm{~h}$. Following this time, the aqueous layer was extracted with $\mathrm{CH}_{2} \mathrm{Cl}_{2}(3 \mathrm{x})$, and the combined organic layers were dried over $\mathrm{Na}_{2} \mathrm{SO}_{4}$. The solvent was filtered and removed in vacuo. Purification by flash chromatography on $\mathrm{SiO}_{2}(5: 1$ hexanes/EtOAc) yielded $95 \mathrm{mg}(60 \%)$ of the product as a clear, viscous oil: IR (thin film) 3364, 3027, 2960, 1679, 1454, 1364, 1054, 969, 758, $700 \mathrm{~cm}^{-1}$; ${ }^{1} \mathrm{H}$ NMR $\left(300 \mathrm{MHz}, \mathrm{CDCl}_{3}\right): \delta 7.33-7.17(\mathrm{~m}, 5 \mathrm{H}), 5.68-5.52(\mathrm{~m}, 2 \mathrm{H}), 4.55(\mathrm{dq}, J=9.6,3.3 \mathrm{~Hz}, 1 \mathrm{H}), 3.79(\mathrm{dt}, J=$ $11.6,2.6 \mathrm{~Hz}, 1 \mathrm{H}), 3.74(\mathrm{dd}, J=10.9,8.4 \mathrm{~Hz}, 1 \mathrm{H}), 3.33(\mathrm{ddd}, J=11.8,8.0,3.8 \mathrm{~Hz}, 1 \mathrm{H}), 3.31(\mathrm{~d}, J=4.1 \mathrm{~Hz}, 1 \mathrm{H})$, $2.95(\mathrm{dd}, J=15.6,9.6 \mathrm{~Hz}, 1 \mathrm{H}), 2.67(\mathrm{dd}, J=15.7,3.3 \mathrm{~Hz}, 1 \mathrm{H}), 2.62(\mathrm{dd}, J=8.0,2.6 \mathrm{~Hz}, 1 \mathrm{H}), 2.03(\mathrm{q}, J=6.7$ $\mathrm{Hz}, 2 \mathrm{H}), 1.64-1.56(\mathrm{~m}, 1 \mathrm{H}), 1.49(\mathrm{~s}, 9 \mathrm{H}), 1.40-1.24(\mathrm{~m}, 4 \mathrm{H}), 0.88(\mathrm{t}, J=7.1 \mathrm{~Hz}, 3 \mathrm{H}) ;{ }^{13} \mathrm{C} \mathrm{NMR}(75 \mathrm{MHz}$, $\left.\mathrm{CDCl}_{3}\right): \delta 200.2,143.6,132.6,131.6,128.6,127.8,126.2,69.8,60.5,50.3,48.5,48.0,47.6,32.2,31.4,29.7$, 22.1, 13.8; MS (EI) $\mathrm{m} / \mathrm{z} 360\left(\mathrm{M}^{+}-\mathrm{H}_{2} \mathrm{O}\right), 304,173,117$, 91; HRMS (EI) $\mathrm{m} / \mathrm{z}$ calculated for $\mathrm{C}_{22} \mathrm{H}_{32} \mathrm{O}_{2} \mathrm{~S}\left(\mathrm{M}^{+}-\mathrm{H}_{2} \mathrm{O}\right)$ : 360.2123 , found 360.2128 .

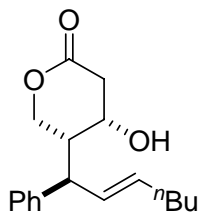

$\boldsymbol{R}^{*}$-4-Hydroxy-5-(1-phenyl-hept-2-enyl)tetrahydropyran-2-one (12): To $0.050 \mathrm{~g}(0.14 \mathrm{mmol})$ of $\beta$-boronic aldehyde 8a (92:8 syn : anti) in $1.4 \mathrm{~mL} \mathrm{CH}_{2} \mathrm{Cl}_{2}$ was added $37 \mathrm{mg}(0.18 \mathrm{mmol})$ of (1tert-butylsulfanyl-vinyloxy)-trimethyl-silane, ${ }^{9}$ and the flask was immersed in a $-78{ }^{\circ} \mathrm{C}$ bath. To the mixture was added $0.21 \mathrm{~mL}(0.21 \mathrm{mmol})$ of dimethylaluminum chloride in hexanes $(1.0 \mathrm{M})$ dropwise and the reaction was stirred for $1 \mathrm{~h}$ at $-78^{\circ} \mathrm{C}$. The reaction was quenched at $-78{ }^{\circ} \mathrm{C}$ by addition of $\mathrm{H}_{2} \mathrm{O}$ and slowly raised to ambient temperature. The aqueous layer was extracted with $\mathrm{Et}_{2} \mathrm{O}(3 \mathrm{x})$ and the combined organic layers were dried over $\mathrm{Na}_{2} \mathrm{SO}_{4}$. Filtration of the organic extracts followed by removal of the solvent in vacuo left a residue that was immediately subject to $4 \mathrm{~mL}$ of a 2:1:1 $\mathrm{MeOH}$ : aq. $1 \mathrm{M} \mathrm{NaOH}: 30 \%$ $\mathrm{HOOH}$ solution for $1 \mathrm{~h}$. Following this time, the solution was acidified with aq. $1 \mathrm{M} \mathrm{HCl}$ to $\sim \mathrm{pH} 0.5$ and stirred for $2 \mathrm{~h}$. The aqueous layer was then extracted with $\mathrm{Et}_{2} \mathrm{O}(3 \mathrm{x})$ and the combined organic layers were dried over $\mathrm{Na}_{2} \mathrm{SO}_{4}$, filtered and the crude product mixture was concentrated in vacuo. Purification of the crude product mixture by flash chromatography on $\mathrm{SiO}_{2}$ (7:3 hexanes/EtOAc) afforded $29 \mathrm{mg}$ (71\%) of the title compound as a clear, viscous oil. Separation of the diastereomers by GC-MS provided the diastereomer ratio: $2.7 \%\left(\mathrm{~T}_{\mathrm{r}}=\right.$ 19.26), 97.3\% $\left(\mathrm{T}_{\mathrm{r}}=19.38\right)$. IR (thin film) 3431, 3028, 2957, 2926, 1720, 1188, 1064, 982, $702 \mathrm{~cm}^{-1} ;{ }^{1} \mathrm{H}$ NMR $\left(300 \mathrm{MHz}, \mathrm{CDCl}_{3}\right): \delta$ 7.36-7.16 (m, 5H), 5.69-5.54 (m, 2H), 4.48-4.35 (m, 1H), $4.32(\mathrm{t}, J=11.5 \mathrm{~Hz}, 1 \mathrm{H}), 3.81$ $(\mathrm{dd}, J=11.6,4.9 \mathrm{~Hz}, 1 \mathrm{H}), 3.25(\mathrm{dd}, J=10.9,8.3 \mathrm{~Hz}, 1 \mathrm{H}), 2.79(\mathrm{dd}, J=18.2,2.9 \mathrm{~Hz}, 1 \mathrm{H}), 2.72(\mathrm{dd}, J=18.2,3.9$ $\mathrm{Hz}, 1 \mathrm{H}), 2.24(\mathrm{tdd}, J=11.6,4.9,1.6 \mathrm{~Hz}, 1 \mathrm{H}), 2.02(\mathrm{q}, J=6.8 \mathrm{~Hz}, 2 \mathrm{H}), 1.36-1.25(\mathrm{~m}, 4 \mathrm{H}), 0.88(\mathrm{t}, J=7.0 \mathrm{~Hz}$,

\footnotetext{
${ }^{9}$ Evans, D. A.; Scheidt, K. A.; Johnston, J. N.; Willis, M. C. J. Am. Chem. Soc. 2001, 123, 4480.
} 
$1 \mathrm{H}) ;{ }^{13} \mathrm{C}$ NMR (75 MHz, $\left.\mathrm{CDCl}_{3}\right): \delta 170.3,141.7,133.1,130.3,128.9,127.2,126.9,68.3,63.4,47.5,41.8,39.3$, 32.1, 31.3, 22.2, 13.8; MS (EI) $\mathrm{m} / \mathrm{z} 270\left(\mathrm{M}^{+}-\mathrm{H}_{2} \mathrm{O}\right), 210,173,117$, 91; HRMS (EI) $\mathrm{m} / \mathrm{z}$ calculated for $\mathrm{C}_{18} \mathrm{H}_{22} \mathrm{O}_{2}$ $\left(\mathrm{M}^{+}-\mathrm{H}_{2} \mathrm{O}\right): 270.1620$, found 270.1623. An X-ray structure determination for a related compound is provided below.

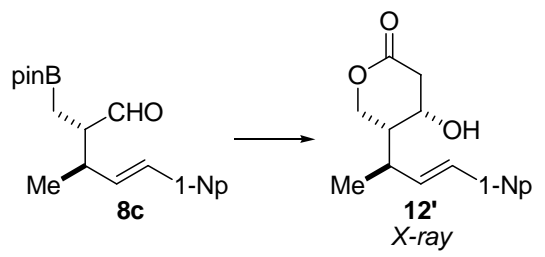

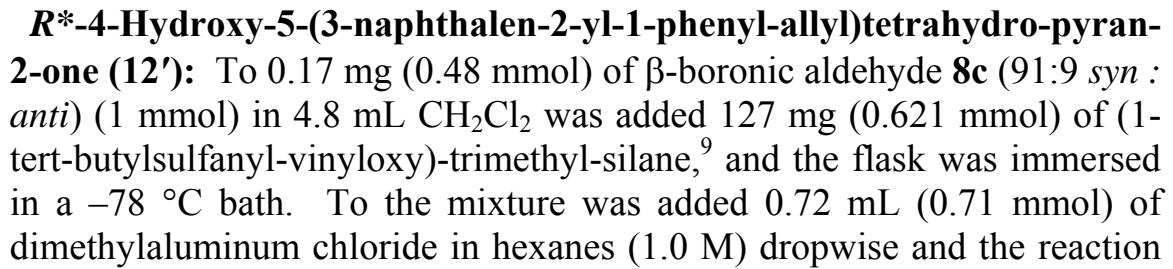
was stirred for $1 \mathrm{~h}$ at $-78^{\circ} \mathrm{C}$. The reaction was quenched at $-78{ }^{\circ} \mathrm{C}$ with $10 \% \mathrm{w} / \mathrm{w}$ citric acid in $\mathrm{MeOH}$ and slowly raised to ambient temperature. Water was added to form a biphasic mixture and the aqueous layer was extracted with $\mathrm{Et}_{2} \mathrm{O}(3 \mathrm{x})$. The combined organic layers were dried over $\mathrm{Na}_{2} \mathrm{SO}_{4}$, filtered and the solvent removed. The crude borane was passed through a plug of silica (5:1 hexanes:EtOAc) and isolated in vacuo. The product mixture was then subject to $12 \mathrm{~mL}$ of a $2: 1: 1 \mathrm{CH}_{2} \mathrm{Cl}_{2}$ : aq. $1 \mathrm{M} \mathrm{NaOH}$ : $30 \% \mathrm{HOOH}$ solution for $1.5 \mathrm{~h}$. Following this time, the aqueous layer was extracted with $\mathrm{CH}_{2} \mathrm{Cl}_{2}(3 \mathrm{x})$, and the organic layers were dried over $\mathrm{Na}_{2} \mathrm{SO}_{4}$. The layers were filtered and the solvent removed in vacuo. The crude product was then treated with 12 $\mathrm{mL} 1 \mathrm{M} \mathrm{NaOH}$ in $\mathrm{MeOH}$ for $1 \mathrm{~h}$ at ambient temperature, then acidified to $\sim \mathrm{pH} 2$ with aq. $1 \mathrm{M} \mathrm{HCl}$ and stirred for an additional $1 \mathrm{~h}$. The reaction was then diluted with water and the aqueous layer was extracted with $\mathrm{Et}_{2} \mathrm{O}(3 \mathrm{x})$. The combined organic layers were dried over $\mathrm{Na}_{2} \mathrm{SO}_{4}$, filtered, and the crude product mixture concentrated in vacuo. Remaining solvents were removed under high vacuum. Purification by flash chromatography on $\mathrm{SiO}_{2}$ (3:2 hexanes/EtOAc) gave $54 \mathrm{mg}(38 \%)$ of the product as a white foam. Recrystallization from hexanes/EtOAc (slow evaporation) gave crystals suitable for X-ray analysis: m.p. 117-119 ${ }^{\circ} \mathrm{C}$; IR (KBr) 3362, 3053, 2965, 1709, 1195, 1041, $971 \mathrm{~cm}^{-1} ;{ }^{1} \mathrm{H}$ NMR $\left(300 \mathrm{MHz}, \mathrm{CDCl}_{3}\right): \delta 7.81-7.43(\mathrm{~m}, 7 \mathrm{H}), 6.66(\mathrm{~d}, J=15.8 \mathrm{~Hz}, 1 \mathrm{H}), 6.21(\mathrm{dd}, J=$ 15.8, $9.3 \mathrm{~Hz}, 1 \mathrm{H}), 4.54(\mathrm{t}, J=11.1 \mathrm{~Hz}, 1 \mathrm{H}), 4.47(\mathrm{dd}, J=10.9,5.7 \mathrm{~Hz}, 1 \mathrm{H}), 4.35-4.30(\mathrm{~m}, 1 \mathrm{H}), 2.73(\mathrm{dd}, J=$ $18.1,2.8 \mathrm{~Hz}, 1 \mathrm{H}), 2.65(\mathrm{dd}, J=18.1,3.7 \mathrm{~Hz}, 1 \mathrm{H}), 2.51$ (tq, $J=9.2,6.8 \mathrm{~Hz}, 1 \mathrm{H}), 1.87(\mathrm{td}, J=9.7,5.9 \mathrm{~Hz}, 1 \mathrm{H})$, $1.18(\mathrm{~d}, J=6.7 \mathrm{~Hz}, 3 \mathrm{H}) ;{ }^{13} \mathrm{C}$ NMR $\left(75 \mathrm{MHz}, \mathrm{CDCl}_{3}\right): \delta 170.1,134.2,133.5,133.0,132.8,130.8,128.2,127.8$, 127.6, 126.3, 125.9 (2C), 123.3, 68.3, 63.9, 42.5, 39.4, 36.0, 18.6; MS (EI) $\mathrm{m} / \mathrm{z} 296\left(\mathrm{M}^{+}\right), 278,181$; HRMS (EI) $\mathrm{m} / \mathrm{z}$ calculated for $\mathrm{C}_{19} \mathrm{H}_{20} \mathrm{O}_{3}\left(\mathrm{M}^{+}\right): 296.1412$, found 296.1401 .<smiles>CC/C=C\C(=O)c1ccccc1C(C)[C@@H](O)CC(=O)[Sn](C)(C)C</smiles>

$\boldsymbol{R}^{*}$-3-Hydroxy-4-methyl-5-phenylnon-6-enethioic acid S-tert-butyl ester: To $0.100 \mathrm{~g}$ $(0.494 \mathrm{mmol})$ of aldehyde $\mathbf{1 3}^{10}$ in $5 \mathrm{~mL} \mathrm{CH}_{2} \mathrm{Cl}_{2}$ was added $131 \mathrm{mg}(0.641 \mathrm{mmol})$ of (1-tertbutylsulfanyl-vinyloxy)-trimethyl-silane, and the flask was immersed in a $-78{ }^{\circ} \mathrm{C}$ bath. To the mixture was added $0.74 \mathrm{~mL}(0.74 \mathrm{mmol})$ of dimethylaluminum chloride in hexanes $(1.0 \mathrm{M})$ dropwise and the reaction was stirred for $1 \mathrm{~h}$ at $-78{ }^{\circ} \mathrm{C}$. The reaction was quenched by addition of $10 \% \mathrm{w} / \mathrm{v}$ citric acid in $\mathrm{MeOH}$ and slowly raised to ambient temperature; stirring was continued for $1 \mathrm{~h}$. The reaction was diluted with water and the aqueous layer was extracted with $\mathrm{Et}_{2} \mathrm{O}(3 \mathrm{x})$. The combined organic layers were dried over $\mathrm{Na}_{2} \mathrm{SO}_{4}$, filtered and the crude product mixture was concentrated in vacuo. Remaining solvents were removed under high vacuum. Purification via flash chromatography on $\mathrm{SiO}_{2}(10: 1$ hexanes/EtOAc) afforded $143 \mathrm{mg}$ $(86 \%)$ of the product as a clear, viscous oil. Separation of the diastereomers by GC-MS provided the diastereomer ratio: $97.8 \%\left(\mathrm{~T}_{\mathrm{r}}=17.70\right), 2.2 \%\left(\mathrm{~T}_{\mathrm{r}}=17.77\right)$ : IR (thin film) 3485, 3026, 2964, 1678, 1454, 1364, $969,753,700 \mathrm{~cm}^{-1} ;{ }^{1} \mathrm{H}$ NMR $\left(300 \mathrm{MHz}, \mathrm{CDCl}_{3}\right): \delta$ 7.32-7.14 (m, 5H), 5.63-5.52 (m, 2H), $4.42(\mathrm{ddd}, J=9.5,3.1$, $2.2 \mathrm{~Hz}, 1 \mathrm{H}), 3.23$ (dd, $J=10.4,8.6 \mathrm{~Hz}, 1 \mathrm{H}), 2.77(\mathrm{dd}, J=15.5,9.5 \mathrm{~Hz}, 1 \mathrm{H}), 2.54(\mathrm{dd}, J=15.5,3.2 \mathrm{~Hz}, 1 \mathrm{H}), 2.03$ $(\mathrm{qd}, J=7.4,4.7 \mathrm{~Hz}, 2 \mathrm{H}), 1.73(\mathrm{ddq}, J=10.5,6.9,2.2 \mathrm{~Hz}, 1 \mathrm{H}), 1.49(\mathrm{~s}, 9 \mathrm{H}), 0.97(\mathrm{t}, J=7.4 \mathrm{~Hz}, 3 \mathrm{H}), 0.68(\mathrm{~d}, J=$ $6.8 \mathrm{~Hz}, 3 \mathrm{H}) ;{ }^{13} \mathrm{C} \mathrm{NMR}\left(75 \mathrm{MHz}, \mathrm{CDCl}_{3}\right): \delta 200.3,144.5,133.4,131.5,128.5,127.8,126.0,68.3,52.8,49.9$, 48.4, 42.4, 29.8, 25.5, 13.7, 10.9; MS (ESI) $\mathrm{m} / \mathrm{z} 357\left(\mathrm{M}^{+}+\mathrm{Na}\right.$ ); HRMS (ESI) $\mathrm{m} / \mathrm{z}$ calculated for $\mathrm{NaC}_{20} \mathrm{H}_{30} \mathrm{O}_{2} \mathrm{~S}$ $\left(\mathrm{M}^{+}+\mathrm{Na}\right): 357.1864$, found 357.1847 .

${ }^{10}$ Nelson, S. G.; Bungard, C. J.; Wang, K. J. Am. Chem. Soc. 2003, 125, 13000. 


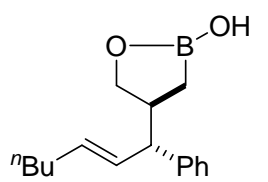

Cyclic borinic acid (14): To a solution of $1.1 \mathrm{~g}(3.1 \mathrm{mmol})$ boronic aldehyde $8 \mathrm{a}$ in $31 \mathrm{~mL}$ pentane at $-78{ }^{\circ} \mathrm{C}$ is slowly added $3.7 \mathrm{~mL}(3.7 \mathrm{mmol}){ }^{i} \mathrm{Bu}_{2} \mathrm{AlH}$ in pentane $(1.0 \mathrm{M})$. The reaction is stirred at $-78{ }^{\circ} \mathrm{C}$ for $30 \mathrm{~min}$, then quenched slowly with $\mathrm{H}_{2} \mathrm{O}$ and warmed to ambient temperature. The cloudy biphasic mixture is extracted with $\mathrm{Et}_{2} \mathrm{O}(3 \mathrm{x})$ and the combined organic extracts are dried over $\mathrm{Na}_{2} \mathrm{SO}_{4}$. (NOTE: emulsions can form in large-scale reductions; $1 \mathrm{M}$ $\mathrm{HCl}$ can be added dropwise until the salts dissolve or a florsil plug can be utilized following extraction to alleviate this problem.) Following filtration, the solvents are removed in vacuo to afford the crude borinic acid. Purification of the product via flash chromatography on $\mathrm{SiO}_{2}(3: 1$ hexanes:EtOAc) afforded $0.46 \mathrm{~g}(55 \%)$ of the product as a clear, viscous oil that was used directly in subsequent transformations.

General Procedure D for Suzuki Cross Coupling Reactions of 14: CEM microwave tubes with snap-on septa were utilized for all coupling reactions and were found to be convenient alternatives to Schlenk tubes for low temperature applications. For reproducible results, it is essential to remove all atmospheric oxygen from the borane/pre-catalyst mixture via high vacuum prior to introduction of the solvent. Degassed solvents are required to give optimal yields for large-scale applications.

A mixture of $11 \mathrm{mg}(0.05 \mathrm{mmol}, 5 \mathrm{~mol} \%)$ of palladium acetate, $39 \mathrm{mg}(0.15 \mathrm{mmol})$ of triphenylphosphine and the borinic acid $14(1.0 \mathrm{mmol})$ are placed in a CEM microwave tube. ${ }^{11}$ The tube is sealed with Teflon tape and the atmosphere is removed under vacuum for $30 \mathrm{~min}$. The reaction vessel is backfilled with nitrogen $3 \mathrm{x}$, following which time $2 \mathrm{~mL}$ of ${ }^{t}$ amyl alcohol is added. ${ }^{12}$ To the stirring solution is immediately added the aryl bromide $(2.1 \mathrm{mmol})$ followed by $0.92 \mathrm{~mL}$ of aq. $1.3 \mathrm{M}$ sodium carbonate. The reaction is stirred for $60 \mathrm{~min}$ at ambient temperature followed by heating at $80^{\circ} \mathrm{C}$ for the indicated period of time (yellow $\rightarrow$ white suspension or clear solution). Upon completion, the reaction is diluted with water, the biphasic mixture is transferred to a seperatory funnel and the aqueous layer is extracted $3 \mathrm{x}$ with EtOAc. The combined organics are dried over $\mathrm{Na}_{2} \mathrm{SO}_{4}$, filtered, and the solvent is removed in vacuo. The crude alcohol is purified as specified. Representative isolated diastereomeric ratios were determined by GC-MS [HP-1 $(12 \mathrm{~m} \mathrm{x} 0.20 \mathrm{~mm})$, pressure $21 \mathrm{kPa}$, method: 70 ${ }^{\circ} \mathrm{C}$ for $2.00 \mathrm{~min}$, ramp $@ 10{ }^{\circ} \mathrm{C} / \mathrm{min}$ to $300{ }^{\circ} \mathrm{C}$, hold for $\left.60 \mathrm{~min}\right]$.

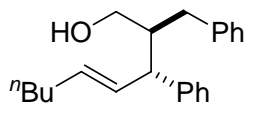

$R^{*}$-(E,2S,3R)-2-Benzyl-3-phenylnon-4-en-1-ol (15a): General Procedure D was followed employing $75 \mathrm{mg}(0.27 \mathrm{mmol})$ of borinic acid $14,3.1 \mathrm{mg}(0.014 \mathrm{mmol})$ of palladium acetate, $11 \mathrm{mg}(0.042 \mathrm{mmol})$ of triphenylphosphine, $0.060 \mathrm{~mL}(0.090 \mathrm{~g}, 0.57 \mathrm{mmol})$ of bromobenzene, $0.25 \mathrm{~mL}$ of aq. $1.3 \mathrm{M}$ sodium carbonate, and a reaction time of $5.5 \mathrm{~h}$. Purification by flash chromatography $(6: 1$ hexanes:EtOAc) on $\mathrm{SiO}_{2}$ gave $54 \mathrm{mg}(67 \%)$ of the title compound as a colorless oil. Separation of the diastereomers by GC-MS provided the diastereomer ratio: $4.5 \%\left(\mathrm{~T}_{\mathrm{r}}=18.80\right), 95.5 \%\left(\mathrm{~T}_{\mathrm{r}}=18.97\right)$ : IR (thin film) 3389, 3026, 2926, 1601, 1494, 1453, 1030, 970, $700 \mathrm{~cm}^{-1}$; ${ }^{1} \mathrm{H}$ NMR $\left(300 \mathrm{MHz}, \mathrm{CDCl}_{3}\right)$ : $\delta$ 7.37-7.09 (m, 10H), $5.69(\mathrm{dd}, J=15.2,8.8 \mathrm{~Hz}, 1 \mathrm{H}), 5.58(\mathrm{dt}, J=15.1,6.1 \mathrm{~Hz}, 1 \mathrm{H}), 3.70(\mathrm{dd}, J=11.2,4.0 \mathrm{~Hz}, 1 \mathrm{H}), 3.55(\mathrm{dd}, J=11.3$, $4.2 \mathrm{~Hz}, 1 \mathrm{H}), 3.34(\mathrm{t}, J=9.1 \mathrm{~Hz}, 1 \mathrm{H}), 2.55(\mathrm{dd}, J=13.8,4.8 \mathrm{~Hz}, 1 \mathrm{H}), 2.46(\mathrm{dd}, J=13.7 .9 .7 \mathrm{~Hz}, 1 \mathrm{H}), 2.14(\mathrm{~m}$, $1 \mathrm{H}), 2.03(\mathrm{q}, J=6.8 \mathrm{~Hz}, 2 \mathrm{H}), 1.33(\mathrm{~m}, 4 \mathrm{H}), 0.88(\mathrm{t}, J=7.0 \mathrm{~Hz}, 3 \mathrm{H}) ;{ }^{13} \mathrm{C}$ NMR $\left(75 \mathrm{MHz}, \mathrm{CDCl}_{3}\right): \delta 144.0,140.9$, 132.0, 131.9, 129.0, 128.6, 128.3, 127.9, 126.2, 125.8, 62.1, 51.2, 47.7, 35.2, 32.2, 31.5, 22.2, 13.9; MS (EI) m/z $308\left(\mathrm{M}^{+}\right), 290,233,199,173,117,91$; HRMS (EI) $\mathrm{m} / \mathrm{z}$ calculated for $\mathrm{C}_{22} \mathrm{H}_{28} \mathrm{O}: 308.2140$, found 308.2147.

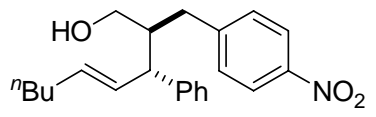

$R^{*}$-(E,2S,3R)-2-(4-Nitrobenzyl)-3-phenylnon-4-en-1-ol (15b): General Procedure D was followed employing $75 \mathrm{mg}(0.27 \mathrm{mmol})$ of borinic acid 14, $3.1 \mathrm{mg}(0.014 \mathrm{mmol})$ of palladium acetate, $11 \mathrm{mg}(0.042 \mathrm{mmol})$ of triphenylphosphine, $0.12 \mathrm{~g}(0.57 \mathrm{mmol})$ of 1-bromo-4-nitrobenzene, $0.25 \mathrm{~mL}$ of aq. $1.3 \mathrm{M}$ sodium carbonate, and a reaction time of $15 \mathrm{~h}$. Purification by flash chromatography (5:1 hexanes:EtOAc) on $\mathrm{SiO}_{2}$ gave $79 \mathrm{mg}(81 \%)$ of the title compound as a colorless oil. Separation of the diastereomers by GC-MS provided the diastereomer ratio: $1.2 \%\left(\mathrm{~T}_{\mathrm{r}}=22.58\right), 98.8 \%\left(\mathrm{~T}_{\mathrm{r}}=\right.$ 22.74): IR (thin film) 3441, 3027, 2927, 1600, 1518, 1345, $700 \mathrm{~cm}^{-1} ;{ }^{1} \mathrm{H}$ NMR $\left(300 \mathrm{MHz}, \mathrm{CDCl}_{3}\right): \delta 8.10(\mathrm{~d}, J=$ $8.8 \mathrm{~Hz}, 2 \mathrm{H}), 7.38-7.21(\mathrm{~m}, 7 \mathrm{H}), 5.66(\mathrm{dd}, J=15.2,8.3 \mathrm{~Hz}, 1 \mathrm{H}), 5.58(\mathrm{dt}, J=15.1,6.0 \mathrm{~Hz}, 1 \mathrm{H}), 3.71(\mathrm{dt}, J=$ $11.1,4.3 \mathrm{~Hz}, 1 \mathrm{H}), 3.50$ (ddd, $J=11.0,5.6,3.9 \mathrm{~Hz}, 1 \mathrm{H}), 3.32(\mathrm{t}, J=9.1 \mathrm{~Hz}, 1 \mathrm{H}), 2.68(\mathrm{dd}, J=13.6,9.5 \mathrm{~Hz}, 1 \mathrm{H})$, $2.58(\mathrm{dd}, J=13.6,4.7 \mathrm{~Hz}, 1 \mathrm{H}), 2.14(\mathrm{~m}, 1 \mathrm{H}), 2.03(\mathrm{q}, J=6.7 \mathrm{~Hz}, 2 \mathrm{H}), 1.31(\mathrm{~m}, 4 \mathrm{H}), 0.88(\mathrm{t}, J=7.3 \mathrm{~Hz}, 3 \mathrm{H}) ;{ }^{13} \mathrm{C}$

\footnotetext{
${ }^{11}$ General procedure: Huff, B. E.; Koenig, T. M.; Mitchell, D.; Staszak, M. A. Org. Synth., Coll. Vol. X 1995, 102.

${ }^{12}$ Solvent system: Kirchoff, J. H.; Netherton, M. R.; Hills, I. D.; Fu, G. C. J. Am. Chem. Soc. 2002, 124, 13662.
} 
NMR (75 MHz, $\left.\mathrm{CDCl}_{3}\right): \delta 149.2,146.4,143.5,132.4,131.5,129.9,128.8,127.8,126.5,123.5,61.5,51.3,47.5$, 35.1, 32.2, 31.5, 22.2, 13.9; MS (EI) $\mathrm{m} / \mathrm{z} 353\left(\mathrm{M}^{+}\right), 278,253,199,174,131,115$; HRMS (EI) $\mathrm{m} / \mathrm{z}$ calculated for $\mathrm{C}_{22} \mathrm{H}_{27} \mathrm{NO}_{3}: 353.1991$, found 353.2002.

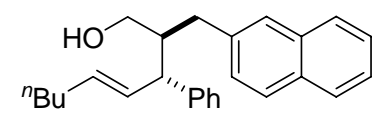

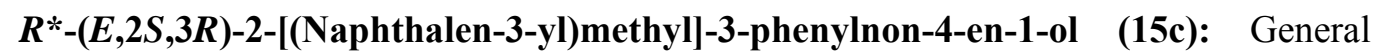
Procedure D was followed employing $75 \mathrm{mg}(0.27 \mathrm{mmol})$ of borinic acid 14, $3.1 \mathrm{mg}$ $(0.014 \mathrm{mmol})$ of palladium acetate, $11 \mathrm{mg}(0.042 \mathrm{mmol})$ of triphenylphosphine, $0.12 \mathrm{~g}$ $(0.57 \mathrm{mmol})$ of 2-bromonapthalene, $0.25 \mathrm{~mL}$ of aq. $1.3 \mathrm{M}$ sodium carbonate, and a reaction time of $1.5 \mathrm{~h}$. Purification by flash chromatography (8:1 hexanes:EtOAc) on $\mathrm{SiO}_{2}$ gave $63 \mathrm{mg}(67 \%)$ of the title compound as a colorless oil. Separation of the diastereomers by GC-MS provided the diastereomer ratio: $1.4 \%\left(\mathrm{~T}_{\mathrm{r}}=23.15\right)$, $98.6 \%\left(\mathrm{~T}_{\mathrm{r}}=23.39\right)$ : IR (thin film) $3382,3025,2926,1600,1452,1028,969,815,747,700 \mathrm{~cm}^{-1} ;{ }^{1} \mathrm{H}$ NMR $(300$ $\left.\mathrm{MHz}, \mathrm{CDCl}_{3}\right): \delta 7.81-7.73(\mathrm{~m}, 3 \mathrm{H}), 7.54(\mathrm{~s}, 1 \mathrm{H}), 7.48-7.21(\mathrm{~m}, 8 \mathrm{H}), 5.71(\mathrm{dd}, J=15.2,9.1 \mathrm{~Hz}, 1 \mathrm{H}), 5.60(\mathrm{dt}, J=$ 15.2, $6.4 \mathrm{~Hz}, 1 \mathrm{H}), 3.73(\mathrm{dt}, J=11.1,5.6 \mathrm{~Hz}, 1 \mathrm{H}), 3.57$ (dt, $J=10.9,5.9 \mathrm{~Hz}, 1 \mathrm{H}), 3.39$ (t, $J=9.0 \mathrm{~Hz}, 1 \mathrm{H}), 2.71$ $(\mathrm{dd}, J=13.7,5.1 \mathrm{~Hz}, 1 \mathrm{H}), 2.64(\mathrm{dd}, J=13.6,9.2 \mathrm{~Hz}, 1 \mathrm{H}), 2.29-2.18(\mathrm{~m}, 1 \mathrm{H}), 2.04(\mathrm{q}, J=6.7 \mathrm{~Hz}, 2 \mathrm{H}), 1.41-1.27$ $(\mathrm{m}, 4 \mathrm{H}), 0.89$ (t, $J=6.9 \mathrm{~Hz}, 3 \mathrm{H}) ;{ }^{13} \mathrm{C} \mathrm{NMR}\left(75 \mathrm{MHz}, \mathrm{CDCl}_{3}\right): \delta 144.0,138.4,133.5,132.1,132.0,131.8,128.6$, $127.9,127.8,127.6,127.5,127.4,127.3,126.3,125.9,125.1,62.0,51.3,47.6,35.3,32.2,31.5,22.2,13.9$; MS (EI) $\mathrm{m} / \mathrm{z} 358\left(\mathrm{M}^{+}\right), 340,283,269,255,199,173,142,117$; HRMS (EI) $\mathrm{m} / \mathrm{z}$ calculated for $\mathrm{C}_{26} \mathrm{H}_{30} \mathrm{O}: 358.2297$, found 358.2300 .

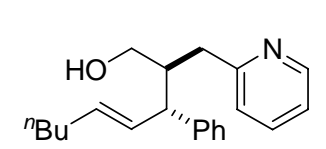

$R^{*}$-(E,2S,3R)-3-Phenyl-2-[(pyridin-2-yl)methyl]non-4-en-1-ol (15d): General Procedure D was followed employing $75 \mathrm{mg}(0.27 \mathrm{mmol})$ of borinic acid $14,3.1 \mathrm{mg}(0.014 \mathrm{mmol})$ of palladium acetate, $11 \mathrm{mg}(0.042 \mathrm{mmol})$ of triphenylphosphine, $0.090 \mathrm{~g}(0.57 \mathrm{mmol})$ of 2 bromopyridine, $0.25 \mathrm{~mL}$ of aq. $1.3 \mathrm{M}$ sodium carbonate, and a reaction time of $48 \mathrm{~h}$. Purification by flash chromatography $\left(2: 1 \rightarrow 1: 1\right.$ hexanes:EtOAc) on $\mathrm{SiO}_{2}$ gave $0.030 \mathrm{~g}(36 \%)$ of the title compound as a light yellow oil: IR (thin film) 3373, 3025, 2925, 1593, 1569, 1472, 969, 756, $701 \mathrm{~cm}^{-1}$; ${ }^{1} \mathrm{H}$ NMR $\left(300 \mathrm{MHz}, \mathrm{CDCl}_{3}\right): \delta 8.48(\mathrm{~d}, J=4.8 \mathrm{~Hz}, 1 \mathrm{H}), 7.55(\mathrm{td}, J=7.7,1.7 \mathrm{~Hz}, 1 \mathrm{H}), 7.35-7.10(\mathrm{~m}, 6 \mathrm{H}), 6.85(\mathrm{~d}, J=7.8$ $\mathrm{Hz}, 1 \mathrm{H}), 5.56(\mathrm{dd}, J=15.2,7.6 \mathrm{~Hz}, 1 \mathrm{H}), 5.47(\mathrm{dt}, J=15.1,5.9 \mathrm{~Hz}, 1 \mathrm{H}), 5.08$ (br. s, $1 \mathrm{H}), 3.69(\mathrm{dd}, J=11.6,4.2$ $\mathrm{Hz}, 1 \mathrm{H}), 3.61(\mathrm{dd}, J=11.6,6.0 \mathrm{~Hz}, 1 \mathrm{H}), 3.21(\mathrm{dd}, J=10.4,8.2 \mathrm{~Hz}, 1 \mathrm{H}), 2.80(\mathrm{dd}, J=14.1,4.2 \mathrm{~Hz}, 1 \mathrm{H}), 2.69$ (dd, $J=14.1,7.8 \mathrm{~Hz}, 1 \mathrm{H}), 2.35$ (dddt, $J=10.3,8.1,6.0,4.2 \mathrm{~Hz}, 1 \mathrm{H}), 1.97$ (q, $J=6.5 \mathrm{~Hz}, 2 \mathrm{H}), 1.35-1.23(\mathrm{~m}, 4 \mathrm{H})$, $0.86(\mathrm{t}, J=6.9 \mathrm{~Hz}, 3 \mathrm{H}) ;{ }^{13} \mathrm{C}$ NMR $\left(75 \mathrm{MHz}, \mathrm{CDCl}_{3}\right): \delta 160.3,148.5,144.1,136.7,132.1,131.7,128.6,128.0$, 126.2, 124.0, 121.2, 63.5, 51.3, 45.0, 38.4, 32.2, 31.5, 22.2, 13.9; MS (EI) m/z $309\left(\mathrm{M}^{+}\right), 278,174,169,136,118$, 106, 91; HRMS (EI) $\mathrm{m} / \mathrm{z}$ calculated for $\mathrm{C}_{21} \mathrm{H}_{27} \mathrm{NO}$ : 309.2093, found 309.2106.

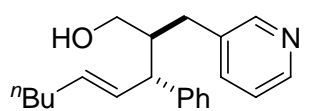

$R^{*}$-(E,2S,3R)-3-Phenyl-2-[(pyridin-3-yl)methyl]non-4-en-1-ol (15e): General Procedure D was followed employing $75 \mathrm{mg}(0.27 \mathrm{mmol})$ of borinic acid $14,3.1 \mathrm{mg}(0.014 \mathrm{mmol})$ of palladium acetate, $11 \mathrm{mg}(0.042 \mathrm{mmol})$ of triphenylphosphine, $55 \mu \mathrm{L}(0.090 \mathrm{~g}, 0.57 \mathrm{mmol})$ of 3-bromopyridine, $0.25 \mathrm{~mL}$ of aq. $1.3 \mathrm{M}$ sodium carbonate, and a reaction time of $20 \mathrm{~h}$. Purification by flash chromatography (1:1 hexanes:EtOAc $\rightarrow 2: 1$ EtOAc:hexanes) on $\mathrm{SiO}_{2}$ gave $66 \mathrm{mg}(78 \%)$ of the title compound as a light yellow oil: IR (thin film) 3276, 3027, 2925, 1597, 1577, 1424, 1029, 968, $702 \mathrm{~cm}^{-1}$; ${ }^{1} \mathrm{H} \mathrm{NMR}(300 \mathrm{MHz}$, $\left.\mathrm{CDCl}_{3}\right): \delta 8.42(\mathrm{dd}, J=4.8,1.5 \mathrm{~Hz}, 1 \mathrm{H}), 8.34(\mathrm{~d}, J=1.9 \mathrm{~Hz}, 1 \mathrm{H}), 7.40(\mathrm{dt}, J=7.8,1.9 \mathrm{~Hz}, 1 \mathrm{H}), 7.37-7.21(\mathrm{~m}$, $5 \mathrm{H}), 7.17(\mathrm{dd}, J=7.8,5.0 \mathrm{~Hz}, 1 \mathrm{H}), 5.66(\mathrm{dd}, J=15.2,8.5 \mathrm{~Hz}, 1 \mathrm{H}), 5.56(\mathrm{dt}, J=15.1,6.2 \mathrm{~Hz}, 1 \mathrm{H}), 3.70(\mathrm{dt}, J=$ $11.2,4.9 \mathrm{~Hz}, 1 \mathrm{H}), 3.53(\mathrm{dt}, J=10.8,4.5 \mathrm{~Hz}, 1 \mathrm{H}), 3.33(\mathrm{t}, J=9.0 \mathrm{~Hz}, 1 \mathrm{H}), 2.59-2.47(\mathrm{~m}, 2 \mathrm{H}), 2.18-2.06(\mathrm{~m}, 1 \mathrm{H})$, $2.02(\mathrm{q}, J=6.7 \mathrm{~Hz}, 2 \mathrm{H}), 1.49(\mathrm{t}, J=5.0 \mathrm{~Hz}, 1 \mathrm{H}), 1.39-1.22(\mathrm{~m}, 4 \mathrm{H}), 0.88(\mathrm{t}, J=6.9 \mathrm{~Hz}, 3 \mathrm{H}) ;{ }^{13} \mathrm{C} \mathrm{NMR}(75 \mathrm{MHz}$, $\left.\mathrm{CDCl}_{3}\right): \delta 150.4,147.2,143.7,136.6,136.4,132.2,131.6,128.7,127.8,126.3,123.2,61.2,50.9,47.3,32.2,32.0$, 31.5, 22.2, 13.9; MS (EI) m/z $309\left(\mathrm{M}^{+}\right), 173,117,91$; HRMS (EI) $\mathrm{m} / \mathrm{z}$ calculated for $\mathrm{C}_{21} \mathrm{H}_{27} \mathrm{NO}$ : 309.2093, found 309.2099 .

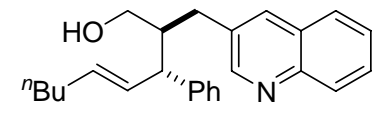

$R^{*}$-(E,2S,3R)-3-Phenyl-2-[(quinolin-3-yl)methyl]non-4-en-1-ol $\quad(15 f): \quad$ General Procedure D was followed employing $75 \mathrm{mg}(0.27 \mathrm{mmol})$ of borinic acid 14, 3.1 $\mathrm{mg}$ $(0.014 \mathrm{mmol})$ of palladium acetate, $11 \mathrm{mg}(0.042 \mathrm{mmol})$ of triphenylphosphine, $78 \mu \mathrm{L}$ $(0.12 \mathrm{~g}, 0.57 \mathrm{mmol})$ of 3-bromoquinoline, $0.25 \mathrm{~mL}$ of aq. $1.3 \mathrm{M}$ sodium carbonate, and a reaction time of $12 \mathrm{~h}$. Purification by flash chromatography (3:2 hexanes:EtOAc) on $\mathrm{SiO}_{2}$ gave $85 \mathrm{mg}(89 \%)$ of the title compound as a 
yellow oil: IR (thin film) 3290, 3026, 2925, 1601, 1574, 1495, 1452, 1034, 967, 787, 752, $701 \mathrm{~cm}^{-1}$; ${ }^{1} \mathrm{H}$ NMR $\left(300 \mathrm{MHz}, \mathrm{CDCl}_{3}\right): \delta 8.66(\mathrm{~d}, J=2.2 \mathrm{~Hz}, 1 \mathrm{H}), 8.07(\mathrm{~d}, J=8.5 \mathrm{~Hz}, 1 \mathrm{H}), 7.83(\mathrm{~d}, J=1.8 \mathrm{~Hz}, 1 \mathrm{H}), 7.73(\mathrm{~d}, J=8.2$ $\mathrm{Hz}, 1 \mathrm{H}), 7.66(\mathrm{ddd}, J=8.4,6.9,1.4 \mathrm{~Hz}, 1 \mathrm{H}), 7.52(\mathrm{ddd}, J=8.0,7.0,1.1 \mathrm{~Hz}, 1 \mathrm{H}), 7.34-7.22(\mathrm{~m}, 5 \mathrm{H}), 5.69(\mathrm{dd}, J$ $=15.2,8.6 \mathrm{~Hz}, 1 \mathrm{H}), 5.59(\mathrm{dt}, J=15.2,5.9 \mathrm{~Hz}, 1 \mathrm{H}), 3.74(\mathrm{dt}, J=11.0,4.8 \mathrm{~Hz}, 1 \mathrm{H}), 3.60(\mathrm{dt}, J=11.0,5.3 \mathrm{~Hz}$, $1 \mathrm{H}), 3.38(\mathrm{t}, J=9.1 \mathrm{~Hz}, 1 \mathrm{H}), 2.76(\mathrm{dd}, J=13.8,8.7 \mathrm{~Hz}, 1 \mathrm{H}), 2.70(\mathrm{dd}, J=14.0,5.2 \mathrm{~Hz}, 1 \mathrm{H}), 2.28-2.18(\mathrm{~m}, 1 \mathrm{H})$, $2.03(\mathrm{q}, J=6.7 \mathrm{~Hz}, 2 \mathrm{H}), 1.60(\mathrm{t}, J=5.4 \mathrm{~Hz}, 1 \mathrm{H}), 1.40-1.23(\mathrm{~m}, 4 \mathrm{H}), 0.88(\mathrm{t}, J=6.9 \mathrm{~Hz}, 3 \mathrm{H}) ;{ }^{13} \mathrm{C} \mathrm{NMR}(75 \mathrm{MHz}$, $\left.\mathrm{CDCl}_{3}\right): \delta 152.3,146.7,143.7,135.2,133.7,132.3,131.6,129.1,128.8,128.6,128.0,127.9,127.3,126.5,126.4$, 61.4, 51.1, 47.4, 32.2 (2C), 31.5, 22.2, 13.9; MS (EI) m/z $359\left(\mathrm{M}^{+}\right), 342,328,262,173,142,117,91$; HRMS (EI) $\mathrm{m} / \mathrm{z}$ calculated for $\mathrm{C}_{25} \mathrm{H}_{29} \mathrm{NO}$ : 359.2249 , found 359.2258 .

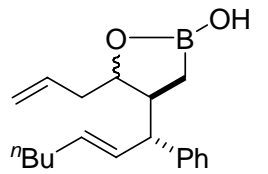

Cyclic homoallylic borinic acid (16): To a solution of $0.96 \mathrm{~g}(2.7 \mathrm{mmol})$ boronic aldehyde 8a in $27 \mathrm{~mL} \mathrm{Et}_{2} \mathrm{O}$ at $-78^{\circ} \mathrm{C}$ is slowly added $3.2 \mathrm{~mL}(3.2 \mathrm{mmol})$ allylmagnesium bromide in $\mathrm{Et}_{2} \mathrm{O}$ (1.0M). The reaction is stirred at $-78^{\circ} \mathrm{C}$ for $1 \mathrm{~h}$, then quenched slowly with $\mathrm{H}_{2} \mathrm{O}$ and warmed to ambient temperature. The cloudy biphasic mixture is extracted with $\mathrm{Et}_{2} \mathrm{O}(3 \mathrm{x})$ and the combined organic extracts are dried over $\mathrm{Na}_{2} \mathrm{SO}_{4}$. Following filtration, the solvents are removed in vacuo to afford the crude boronic alcohol. Purification of the product via flash chromatography on $\mathrm{SiO}_{2} \quad(4: 1$ hexanes:EtOAc) afforded $0.67 \mathrm{~g}(78 \%)$ of the product as a clear, viscous oil.

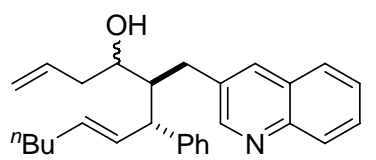

$R^{*}$-(E,4R,5S,6R)-6-Phenyl-5-[(quinolin-3-yl)methyl]dodeca-1,7-dien-4-ol $\quad+\quad R^{*}$ $(E, 4 S, 5 S, 6 R)-6-p h e n y l-5-[(q u i n o l i n-3-y l) m e t h y l] d o d e c a-1,7-d i e n-4-o l$ (17): General Procedure D was followed employing $0.75 \mathrm{~g}(2.4 \mathrm{mmol})$ of borinic acid 16, $27 \mathrm{mg}(0.12$ $\mathrm{mmol})$ of palladium acetate, $94 \mathrm{mg}(0.36 \mathrm{mmol})$ of triphenylphosphine, $0.65 \mathrm{~mL}(1.0 \mathrm{~g}$, $5.0 \mathrm{mmol}$ ) of 3-bromoquinoline, $2.2 \mathrm{~mL}$ of aq. $1.3 \mathrm{M}$ sodium carbonate, and a reaction time of $7 \mathrm{~h}$. Purification by flash chromatography $\left(2: 1\right.$ hexanes:EtOAc) on $\mathrm{SiO}_{2}$ gave $0.54 \mathrm{~g}(58 \%)$ of the title compound as a yellow oil (d.r. 2:1 by $300 \mathrm{MHz}{ }^{1} \mathrm{H}-\mathrm{NMR}$, aryl C ): Diastereomer $A$ - IR (thin film) 3336, 3062, 2955, 1639, 1600, 1573, 1495, 1451, 1049, $750 \mathrm{~cm}^{-1}$; ${ }^{1} \mathrm{H}$ NMR $\left(300 \mathrm{MHz}, \mathrm{CDCl}_{3}\right): \delta 8.52(\mathrm{~d}, J=2.1 \mathrm{~Hz}, 1 \mathrm{H}), 8.03(\mathrm{~d}, J=8.3 \mathrm{~Hz}, 1 \mathrm{H})$, 7.65-7.58 (m, 3H), $7.48(\mathrm{t}, J=7.7 \mathrm{~Hz}, 1 \mathrm{H}), 7.22-7.09(\mathrm{~m}, 5 \mathrm{H}), 5.84-5.76(\mathrm{~m}, 1 \mathrm{H}), 5.70(\mathrm{dd}, J=15.0,9.3 \mathrm{~Hz}, 1 \mathrm{H})$, $5.53(\mathrm{dt}, J=14.9,6.5 \mathrm{~Hz}, 1 \mathrm{H}), 5.17(\mathrm{~d}, J=11.7 \mathrm{~Hz}, 1 \mathrm{H}), 5.16(\mathrm{~d}, J=14.5 \mathrm{~Hz}, 1 \mathrm{H}), 3.95-3.88(\mathrm{~m}, 1 \mathrm{H}), 3.39(\mathrm{t}, J$ $=9.0 \mathrm{~Hz}, 1 \mathrm{H}), 2.98(\mathrm{dd}, J=14.3,6.0 \mathrm{~Hz}, 1 \mathrm{H}), 2.61(\mathrm{dd}, J=14.2,6.7 \mathrm{~Hz}, 1 \mathrm{H}), 2.53-2.42(\mathrm{~m}, 2 \mathrm{H}), 2.30-2.18(\mathrm{~m}$, $1 \mathrm{H}), 2.02(\mathrm{q}, J=6.7 \mathrm{~Hz}, 2 \mathrm{H}), 1.68(\mathrm{~d}, J=4.3 \mathrm{~Hz}, 1 \mathrm{H}), 1.39-1.26(\mathrm{~m}, 4 \mathrm{H}), 0.89(\mathrm{t}, J=7.0 \mathrm{~Hz}, 3 \mathrm{H}) ;{ }^{13} \mathrm{C}$ NMR $(75$ $\left.\mathrm{MHz}, \mathrm{CD}_{3} \mathrm{CN}\right): \delta 153.3,147.4,145.6,137.0,136.6,135.5,133.4,132.9,129.7,129.3(2 \mathrm{C}), 129.2,128.9,128.4$, 127.3, 127.0, 117.2, 71.3, 53.2, 49.9, 41.6, 32.9, 32.4, 31.2, 22.9, 14.2; MS (EI) $\mathrm{m} / \mathrm{z} 399\left(\mathrm{M}^{+}\right), 381,358,340,191$, 173, 142, 117; HRMS (EI) $\mathrm{m} / \mathrm{z}$ calculated for $\mathrm{C}_{28} \mathrm{H}_{33} \mathrm{NO}$ : 399.2570 , found 399.2562 .

Diastereomer B - ${ }^{1} \mathrm{H}$ NMR $\left(300 \mathrm{MHz}, \mathrm{CDCl}_{3}\right): \delta 8.52(\mathrm{~d}, J=2.1 \mathrm{~Hz}, 1 \mathrm{H}), 8.03(\mathrm{~d}, J=8.3 \mathrm{~Hz}, 1 \mathrm{H}), 7.65-7.58(\mathrm{~m}$, $3 \mathrm{H}), 7.48(\mathrm{t}, J=7.7 \mathrm{~Hz}, 1 \mathrm{H}), 7.22-7.09(\mathrm{~m}, 5 \mathrm{H}), 5.84-5.76(\mathrm{~m}, 1 \mathrm{H}), 5.70(\mathrm{dd}, J=15.0,9.3 \mathrm{~Hz}, 1 \mathrm{H}), 5.53(\mathrm{dt}, J=$ 14.9, $6.5 \mathrm{~Hz}, 1 \mathrm{H}), 5.17(\mathrm{~d}, J=11.7 \mathrm{~Hz}, 1 \mathrm{H}), 5.16(\mathrm{~d}, J=14.5 \mathrm{~Hz}, 1 \mathrm{H}), 3.95-3.88(\mathrm{~m}, 1 \mathrm{H}), 3.39(\mathrm{t}, J=9.0 \mathrm{~Hz}$, $1 \mathrm{H}), 2.98(\mathrm{dd}, J=14.3,6.0 \mathrm{~Hz}, 1 \mathrm{H}), 2.61(\mathrm{dd}, J=14.2,6.7 \mathrm{~Hz}, 1 \mathrm{H}), 2.53-2.42(\mathrm{~m}, 2 \mathrm{H}), 2.30-2.18(\mathrm{~m}, 1 \mathrm{H}), 2.02$ $(\mathrm{q}, J=6.7 \mathrm{~Hz}, 2 \mathrm{H}), 1.68(\mathrm{~d}, J=4.3 \mathrm{~Hz}, 1 \mathrm{H}), 1.39-1.26(\mathrm{~m}, 4 \mathrm{H}), 0.89(\mathrm{t}, J=7.0 \mathrm{~Hz}, 3 \mathrm{H}) ; \mathrm{MS}(\mathrm{EI}) \mathrm{m} / \mathrm{z} 399\left(\mathrm{M}^{+}\right)$, $381,358,340,191,173,142,117$; HRMS (EI) $\mathrm{m} / \mathrm{z}$ calculated for $\mathrm{C}_{28} \mathrm{H}_{33} \mathrm{NO}: 399.2610$, found 399.2562 .

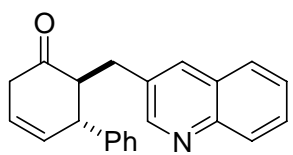

$R^{*}$-(5R,6S)-5-phenyl-6-[(quinolin-3-yl)methyl]cyclohex-3-enone (19): To a solution of 32

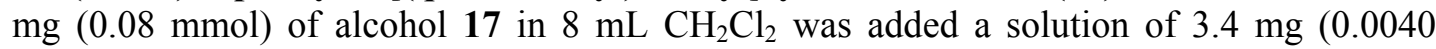
mmol) Grubbs II catalyst (18) in $8 \mathrm{~mL} \mathrm{CH}_{2} \mathrm{Cl}_{2}$ via cannula. The reaction was stirred at ambient temperature for $3 \mathrm{~h}$, then quenched with $15 \mu \mathrm{L}$ of $\mathrm{DMSO}^{13}$ and left for $12 \mathrm{~h}$. The crude reaction mixture was concentrated and the residue was purified by flash chromatography on $\mathrm{SiO}_{2}(2: 1$ EtOAc:hexanes). The purified RCM product was immediately oxidized using $51 \mathrm{mg}(0.12 \mathrm{mmol})$ of Dess-Martin periodinane in $\mathrm{CH}_{2} \mathrm{Cl}_{2}(1 \mathrm{~mL})$ for $30 \mathrm{~min}\left(0^{\circ} \mathrm{C} \rightarrow \mathrm{rt}\right) .^{14}$, The crude ketone is passed through a plug of florsil (1:1 hexanes:EtOAc eluent) to remove heterogeneous impurities and the filtrate was concentrated. Purifying the

\footnotetext{
${ }^{13}$ Ahn, Y. M.; Yang, K.; Georg, G. I. Org. Lett. 2001, 3, 1411.

${ }^{14}$ Dess, D. B.; Martin, J. C. J. Org. Chem. 1983, 48, 4155.

${ }^{15}$ Boeckman Jr., R. K; Shao, P.; Mullins, J. J. Org. Synth., Coll. Vol. X 1995, 696.
} 
crude product mixture by flash chromatography on $\mathrm{SiO}_{2}(2: 1$ hexanes:EtOAc) gave $12 \mathrm{mg}(48 \%)$ of the title compound as a viscous yellow oil: IR (thin film) 3029, 2924, 1716, 1678, 1602, 1571, 1494, 787, 751, $702 \mathrm{~cm}^{-1}$; ${ }^{1} \mathrm{H}$ NMR $\left(300 \mathrm{MHz}, \mathrm{CDCl}_{3}\right): \delta 8.52(\mathrm{~d}, J=1.5 \mathrm{~Hz}, 1 \mathrm{H}), 8.04(\mathrm{~d}, J=8.5 \mathrm{~Hz}, 1 \mathrm{H}), 7.84(\mathrm{~d}, J=1.3 \mathrm{~Hz}, 1 \mathrm{H}), 7.71$ $(\mathrm{d}, J=8.0 \mathrm{~Hz}, 1 \mathrm{H}), 7.64(\mathrm{td}, J=7.0,1.4 \mathrm{~Hz}, 1 \mathrm{H}), 7.50(\mathrm{t}, J=7.8 \mathrm{~Hz}, 1 \mathrm{H}), 7.41-7.22(\mathrm{~m}, 5 \mathrm{H}), 5.87-5.82(\mathrm{~m}, 2 \mathrm{H})$, $3.62(\mathrm{dt}, J=9.5,2.4 \mathrm{~Hz}, 1 \mathrm{H}), 3.27(\mathrm{dd}, J=13.8,8.7 \mathrm{~Hz}, 1 \mathrm{H}), 3.15-3.09(\mathrm{~m}, 2 \mathrm{H}), 2.95(\mathrm{dm}, J=\sim 15.8 \mathrm{~Hz}, 1 \mathrm{H})$, $2.75(\mathrm{dd}, J=13.8,3.3 \mathrm{~Hz}, 1 \mathrm{H}) ;{ }^{13} \mathrm{C} \mathrm{NMR}\left(75 \mathrm{MHz}, \mathrm{CDCl}_{3}\right): \delta 208.4,152.0,146.5,142.3,135.7,133.1,131.2$, 129.1, 128.8 (3C), 128.0, 127.4 (2C), 126.6, 124.1, 57.5, 50.5, 40.5, 30.2; MS (EI) m/z $313\left(\mathrm{M}^{+}\right), 222,182,143$, 130, 115; HRMS (EI) m/z calculated for $\mathrm{C}_{22} \mathrm{H}_{19} \mathrm{NO}$ : 313.1467, found 313.1464. 


\section{X-ray Structure Determinations for Compounds 8c and 12'}

\section{Compound 8c}

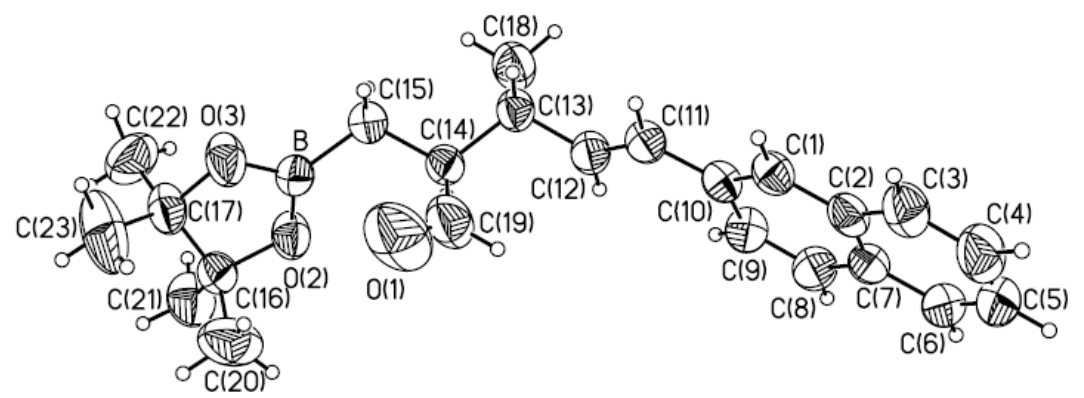

Figure S1. $R^{*}$-(E,2R,3S)-2-[(4,4,5,5-Tetramethyl-1,3,2-dioxaborolan-2-yl)methyl]-3-phenylnon-4-enal (8c)

Table 1. Crystal data and structure refinement for compound $\mathbf{8 c}$.

Empirical formula

Formula weight

Temperature

Wavelength

Crystal system

Space group

Unit cell dimensions

Volume

Z

Density (calculated)

Absorption coefficient

$\mathrm{F}(000)$

Crystal size

Theta range for data collection

Index ranges

Reflections collected

Independent reflections

Completeness to theta $=27.50^{\circ}$

Absorption correction

Max. and min. transmission

Refinement method

Data / restraints / parameters

Goodness-of-fit on $\mathrm{F}^{2}$

Final R indices [I $>2 \operatorname{sigma}(\mathrm{I})]$

$\mathrm{R}$ indices (all data)

Largest diff. peak and hole
$\mathrm{C}_{23} \mathrm{H}_{29} \mathrm{BO}_{3}$

364.27

295(2) K

$0.71073 \AA$

Orthorhombic

Pca2(1)

$\mathrm{a}=12.5986(6) \AA \quad \alpha=90^{\circ}$.

$\mathrm{b}=8.2876(4) \AA \quad \beta=90^{\circ}$.

$\mathrm{c}=20.2572(10) \AA \quad \gamma=90^{\circ}$.

2115.10(18) $\AA^{3}$

4

$1.144 \mathrm{Mg} / \mathrm{m}^{3}$

$0.073 \mathrm{~mm}^{-1}$

784

$0.29 \times 0.21 \times 0.21 \mathrm{~mm}^{3}$

2.01 to $27.50^{\circ}$.

$-16<=\mathrm{h}<=16,-10<=\mathrm{k}<=10,-26<=\mathrm{l}<=26$

19669

$2500[\mathrm{R}($ int $)=0.0238]$

$100.0 \%$

Semi-empirical from equivalents

0.9848 and 0.9791

Full-matrix least-squares on $\mathrm{F}^{2}$

$2500 / 1 / 249$

1.247

$\mathrm{R} 1=0.0481, \mathrm{wR} 2=0.1299$

$\mathrm{R} 1=0.0567, \mathrm{wR} 2=0.1360$

0.224 and -0.127 e. $\AA^{-3}$ 


\section{Compound 12'}

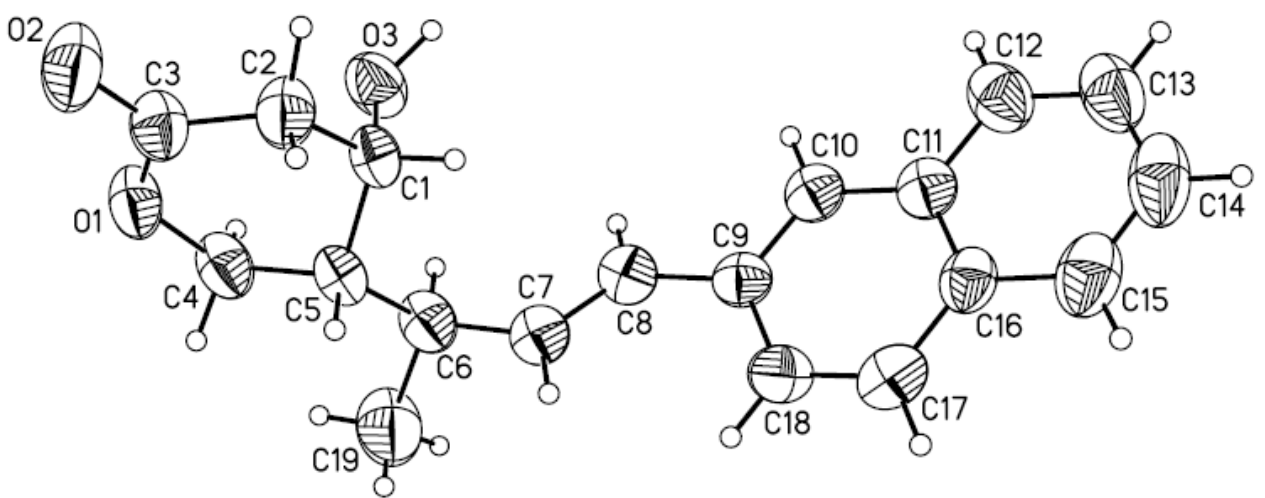

Figure S2. $R^{*}-(4 S, 5 R)$-Tetrahydro-4-hydroxy-5-[(R,E)-4-(naphthalen-3-yl)but-3-en-2-yl]pyran-2-one (12')

Table 2. Crystal data and structure refinement for compound $\mathbf{1 2}^{\prime}$.

Empirical formula

Formula weight

Temperature

Wavelength

Crystal system

Space group

Unit cell dimensions

Volume

Z

Density (calculated)

Absorption coefficient

$\mathrm{F}(000)$

Crystal size

Theta range for data collection

Index ranges

Reflections collected

Independent reflections

Completeness to theta $=25.00^{\circ}$

Absorption correction

Max. and min. transmission

Refinement method

Data / restraints / parameters

Goodness-of-fit on $\mathrm{F}^{2}$

Final R indices [I $>2 \operatorname{sigma}(\mathrm{I})]$

$\mathrm{R}$ indices (all data)

Largest diff. peak and hole

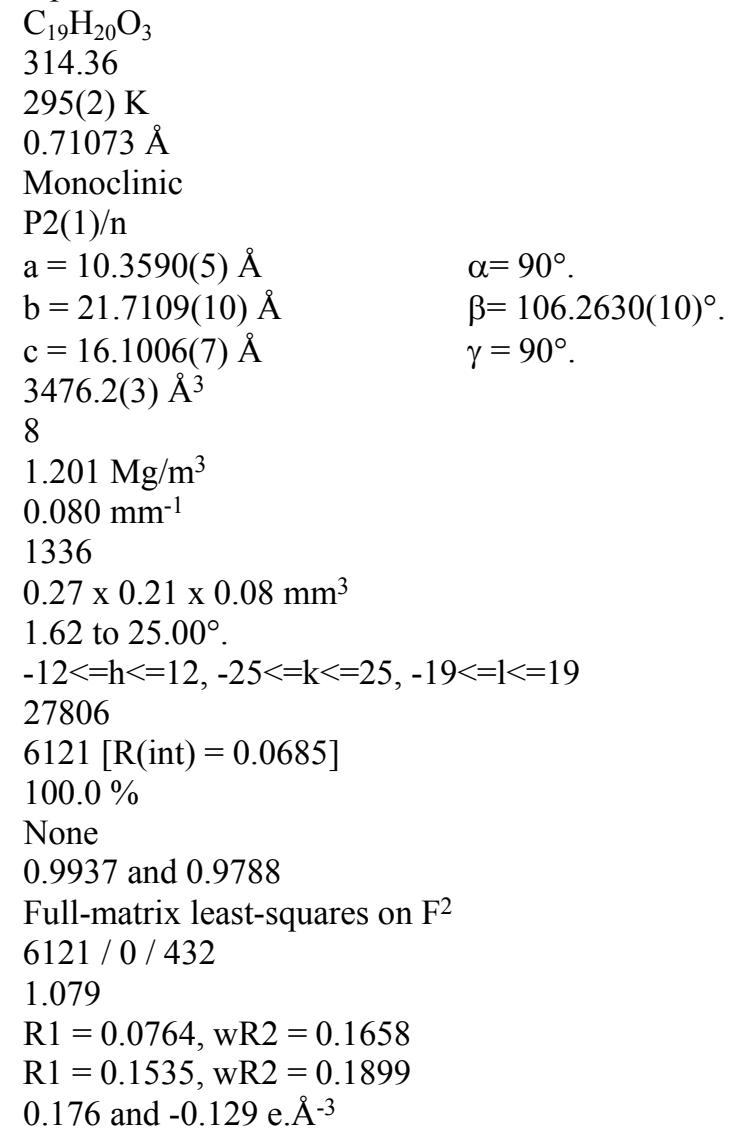




\section{${ }^{1}$ H-NMR Study of $\mathrm{LiO}^{n} \mathrm{Pr}$ Addition to $\beta$-Boronic Aldehyde 8a}

*Note: Spectra taken in $\mathrm{d}_{8}$-THF at high concentration $(\sim 45 \mathrm{mg} / 1 \mathrm{~mL})$, hence chemical shifts were not standardized.

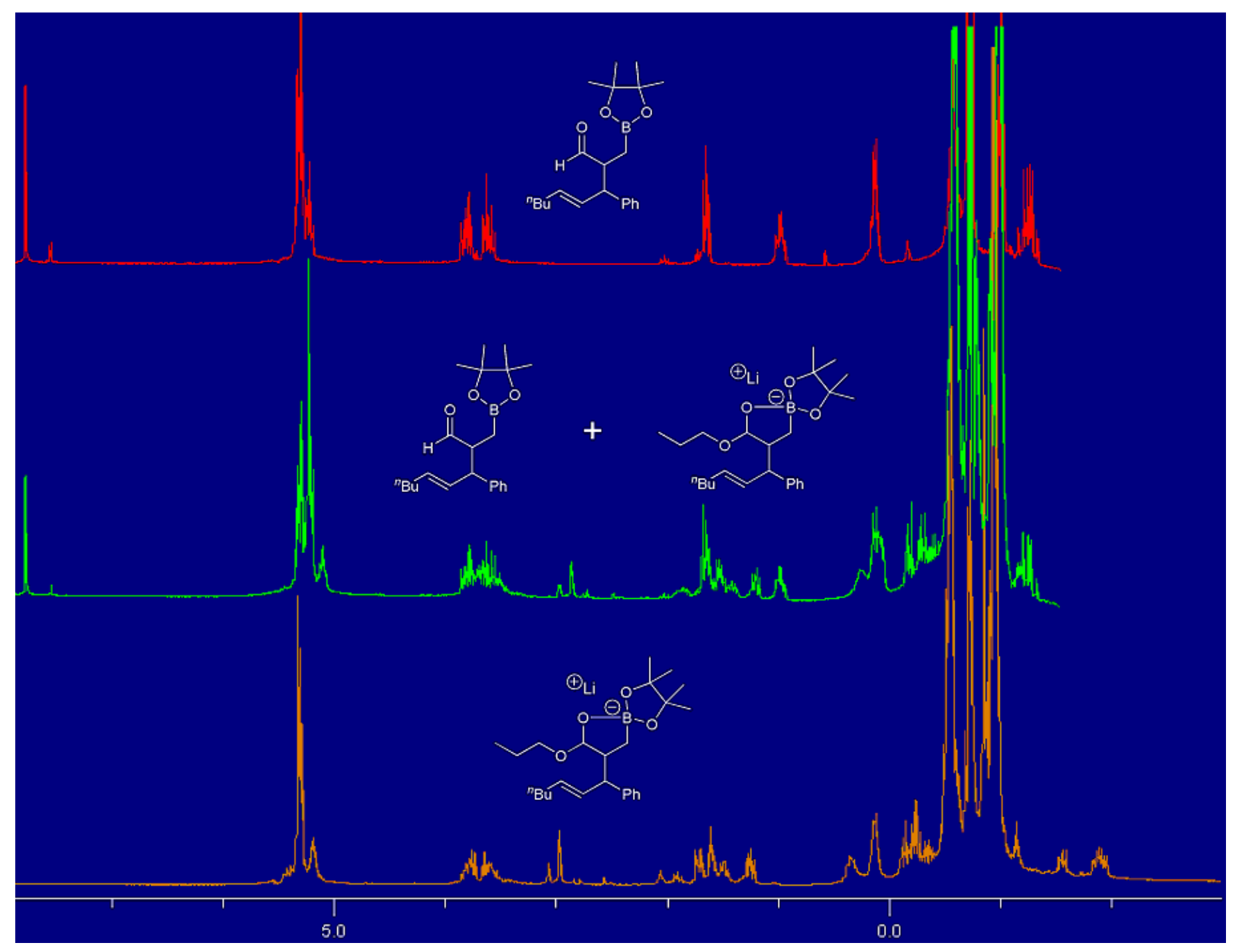


Nelson, S. G., Bungard, C. J. and Stevens, B. D.

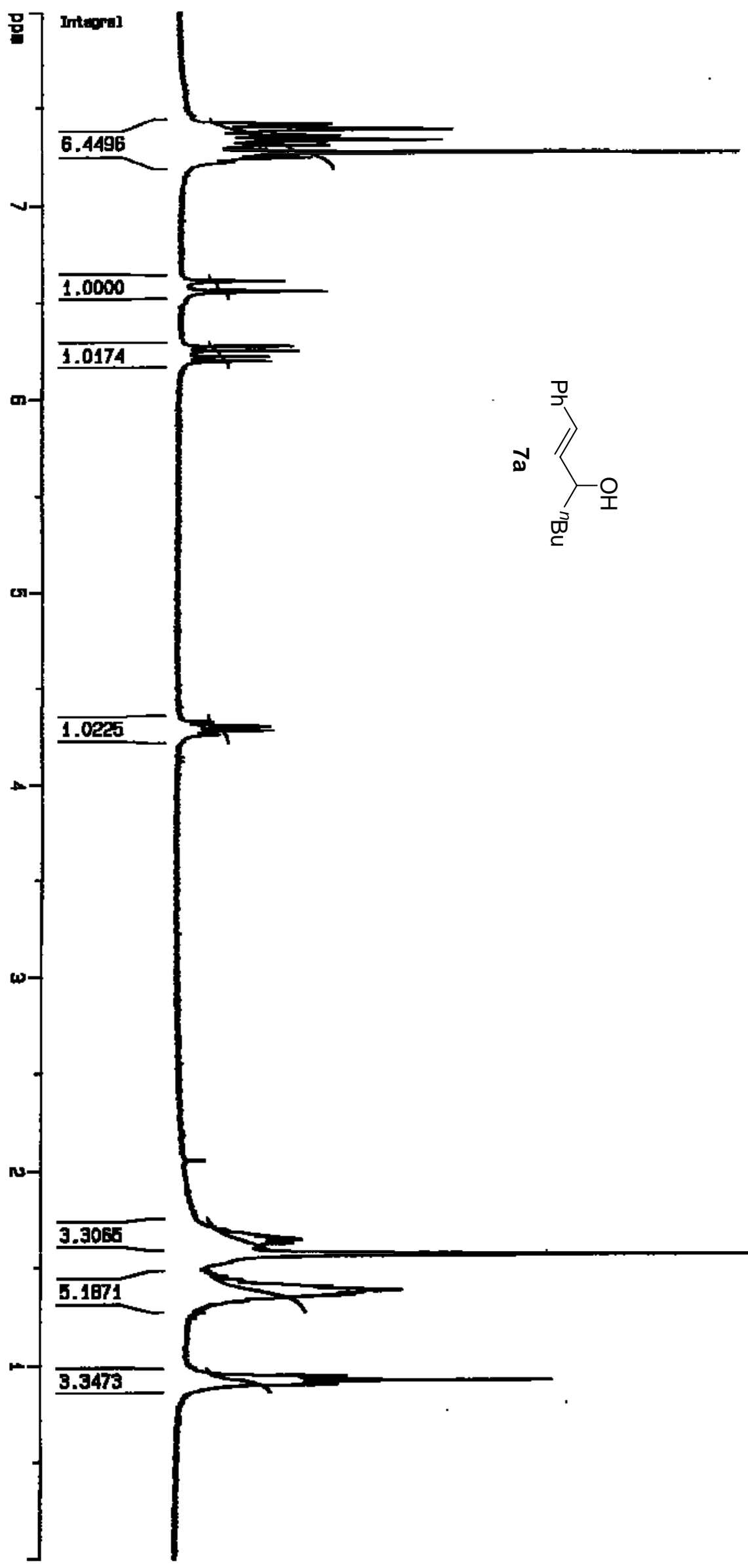

Supporting Information

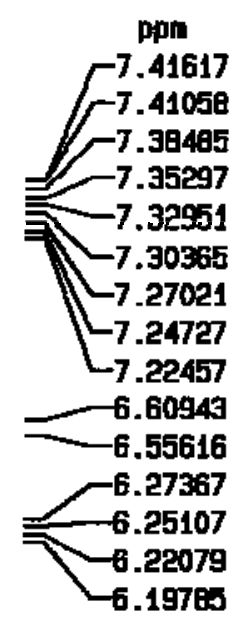




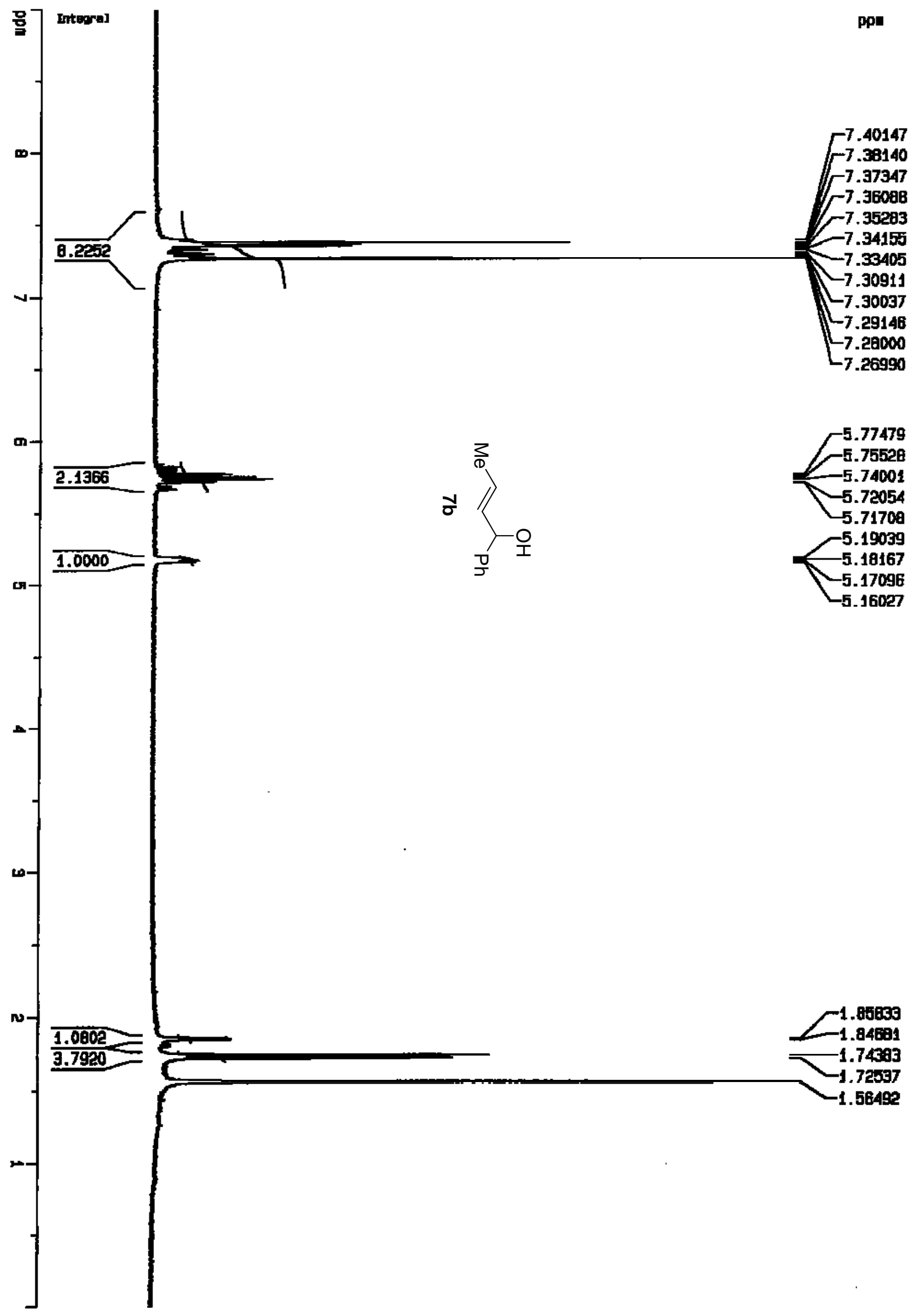


Nelson, S. G., Bungard, C. J. and Stevens, B. D.

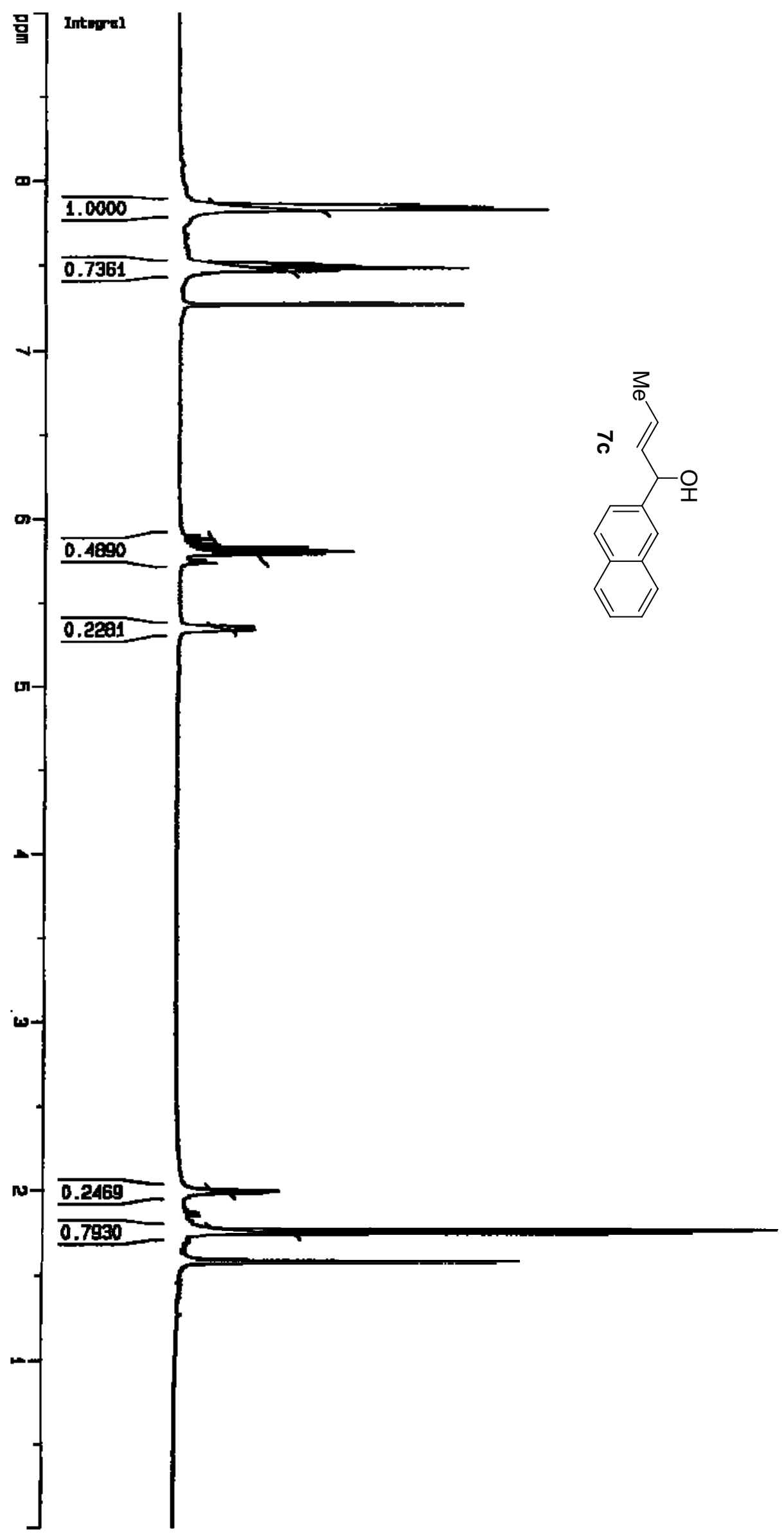

Supporting Information

ppm
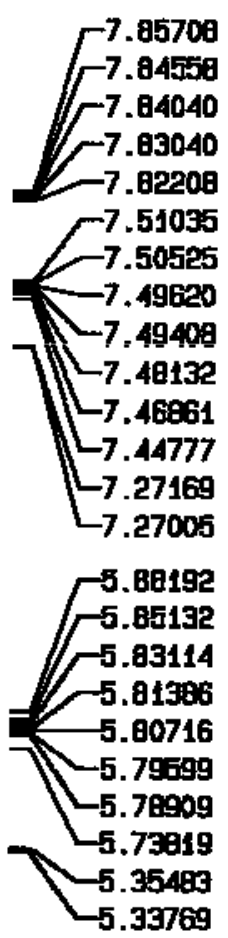


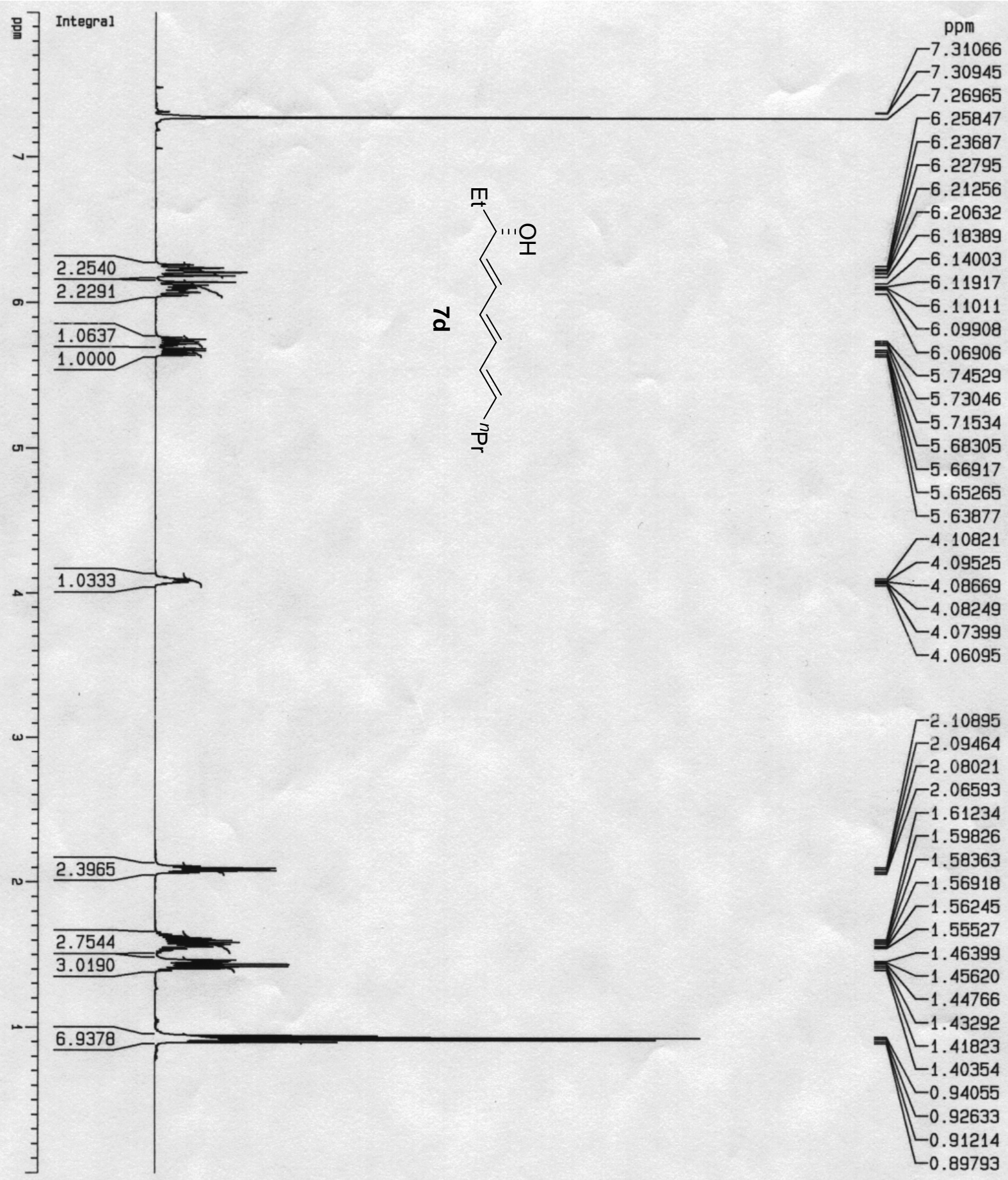

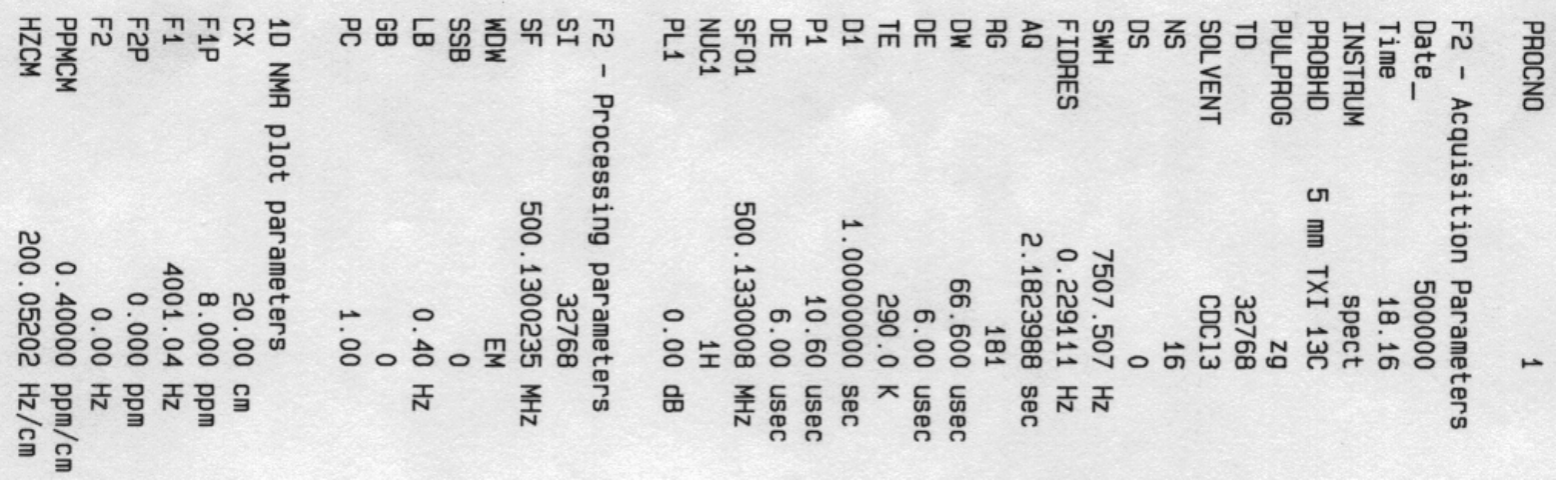


Nelson, S. G., Bungard, C. J. and Stevens, B. D.

Supporting Information

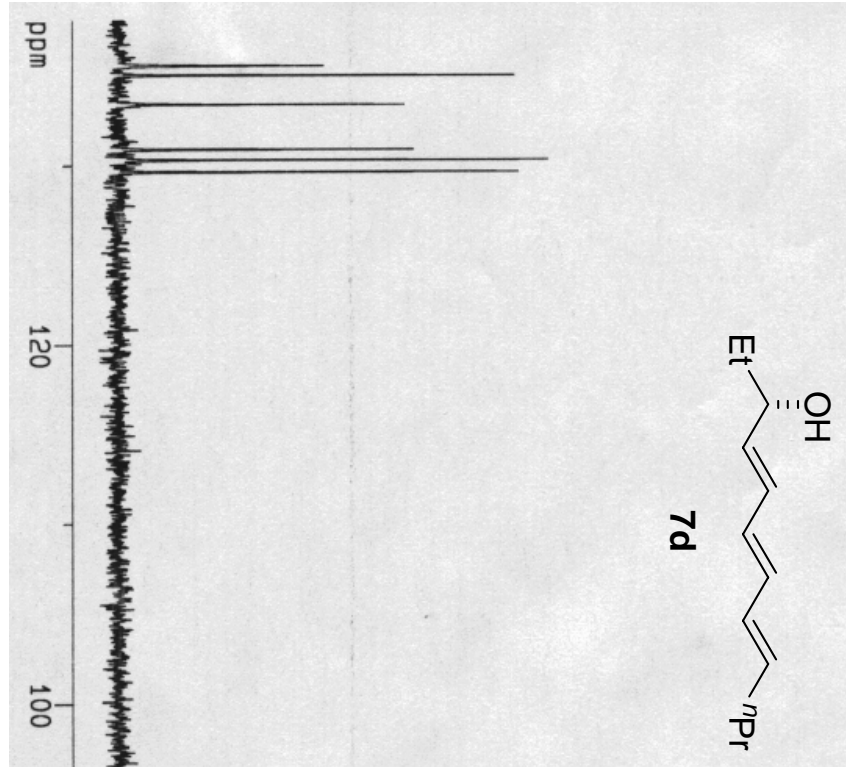

ppm

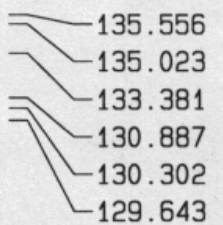

$-129.643$

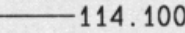

77.427

77.201

77.000

76.579

74.038

용-

o-

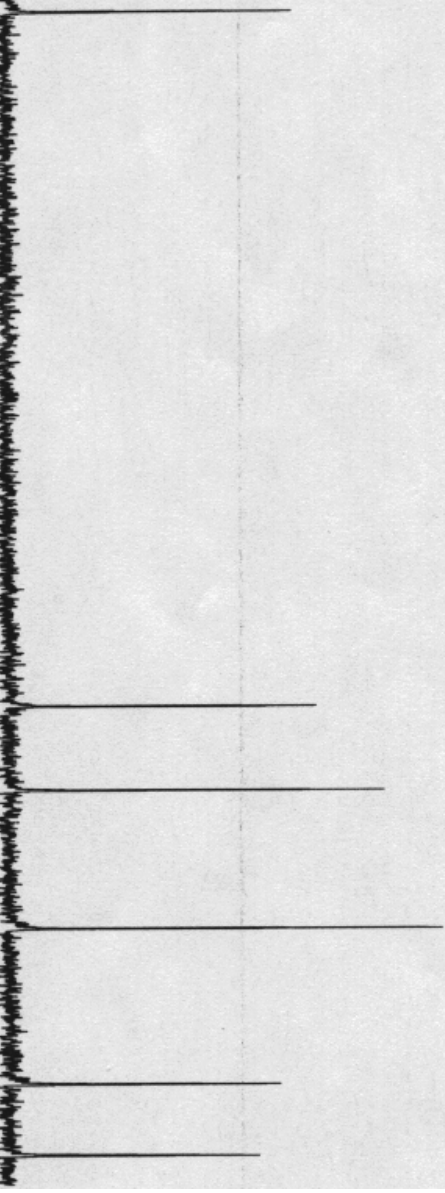

34.833

30.122

22. 381

13.607

9.622 


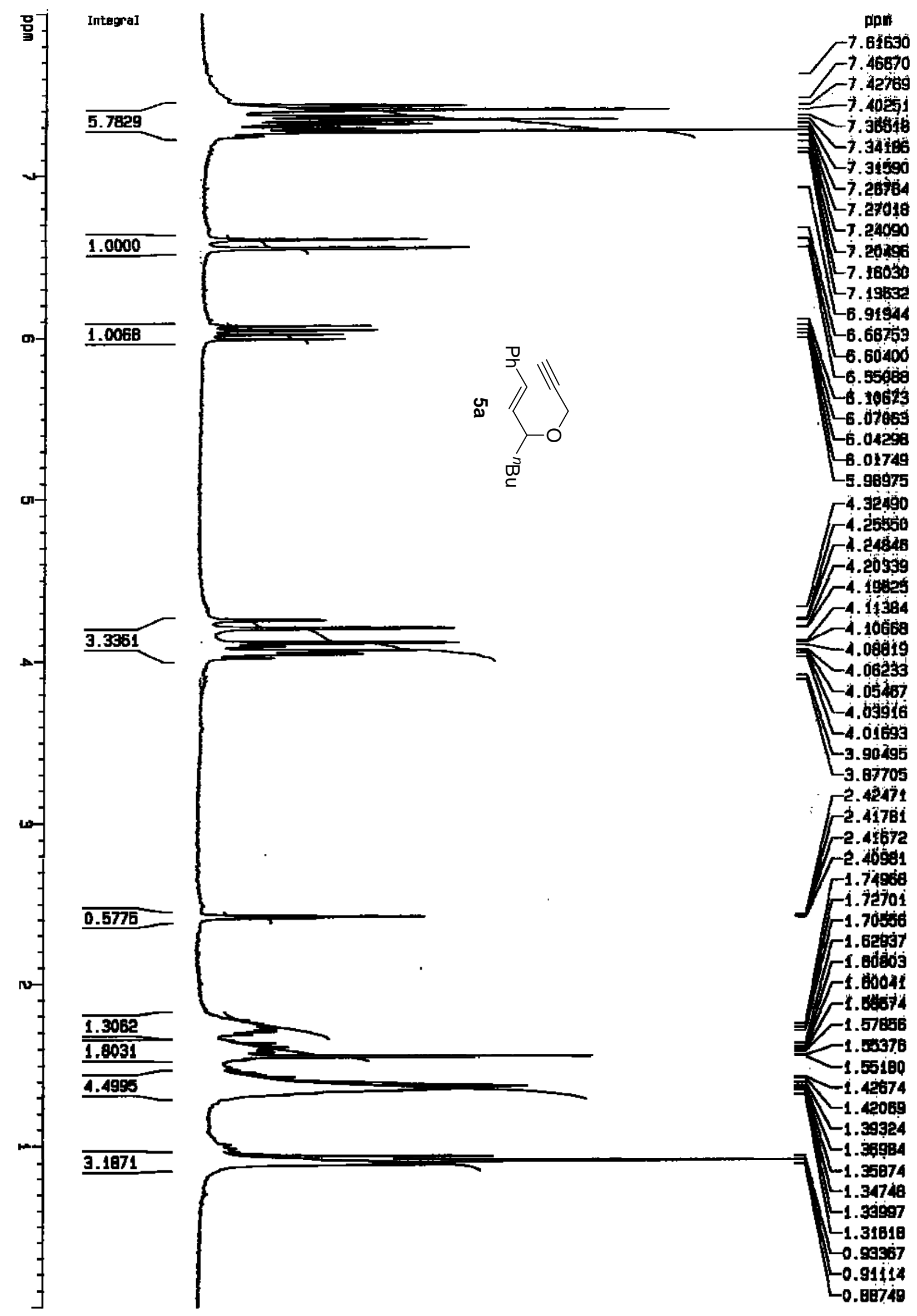


Nelson, S. G., Bungard, C. J. and Stevens, B. D.

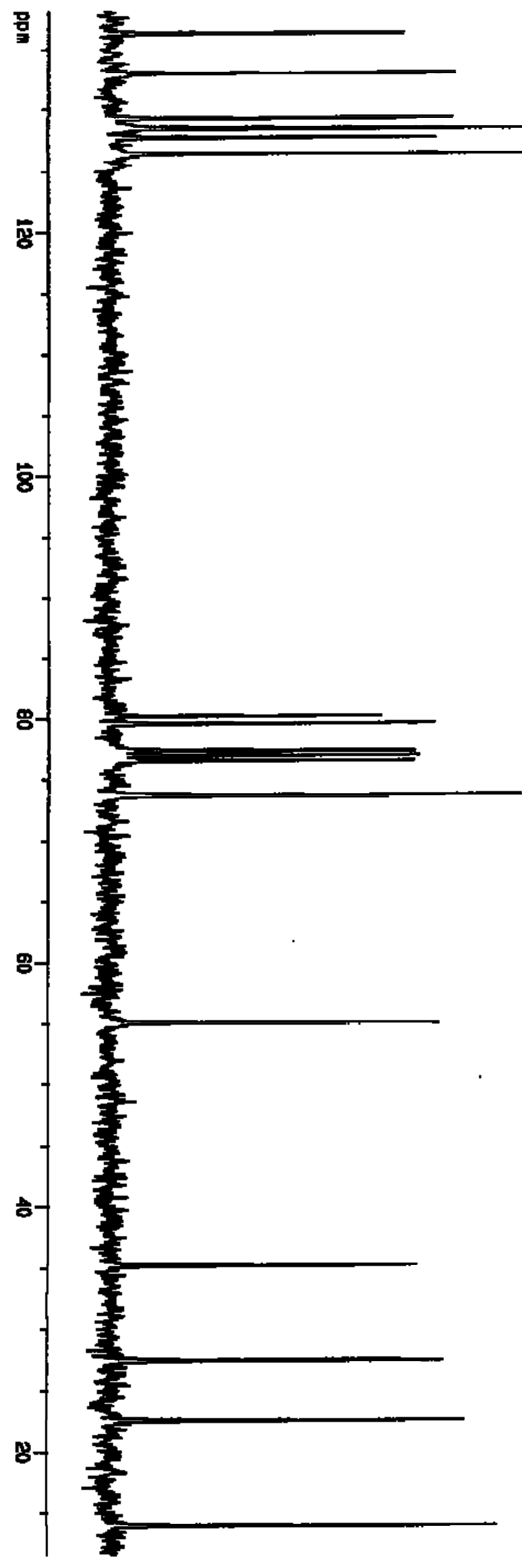

Supporting Information
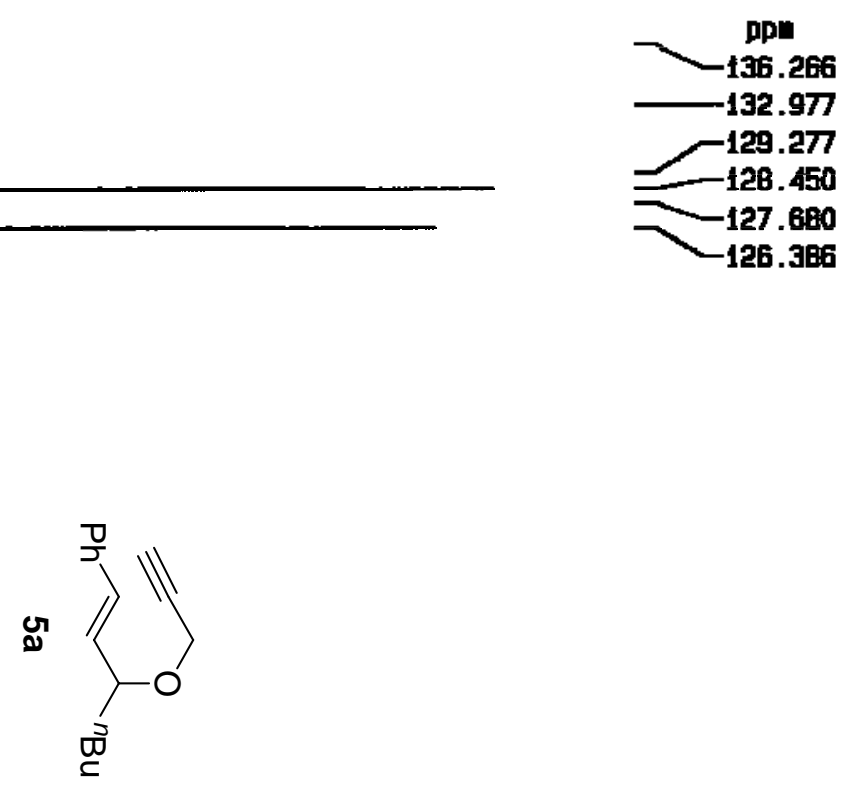

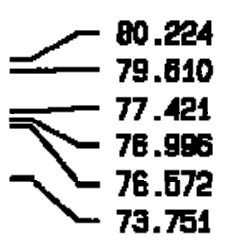

56.019 

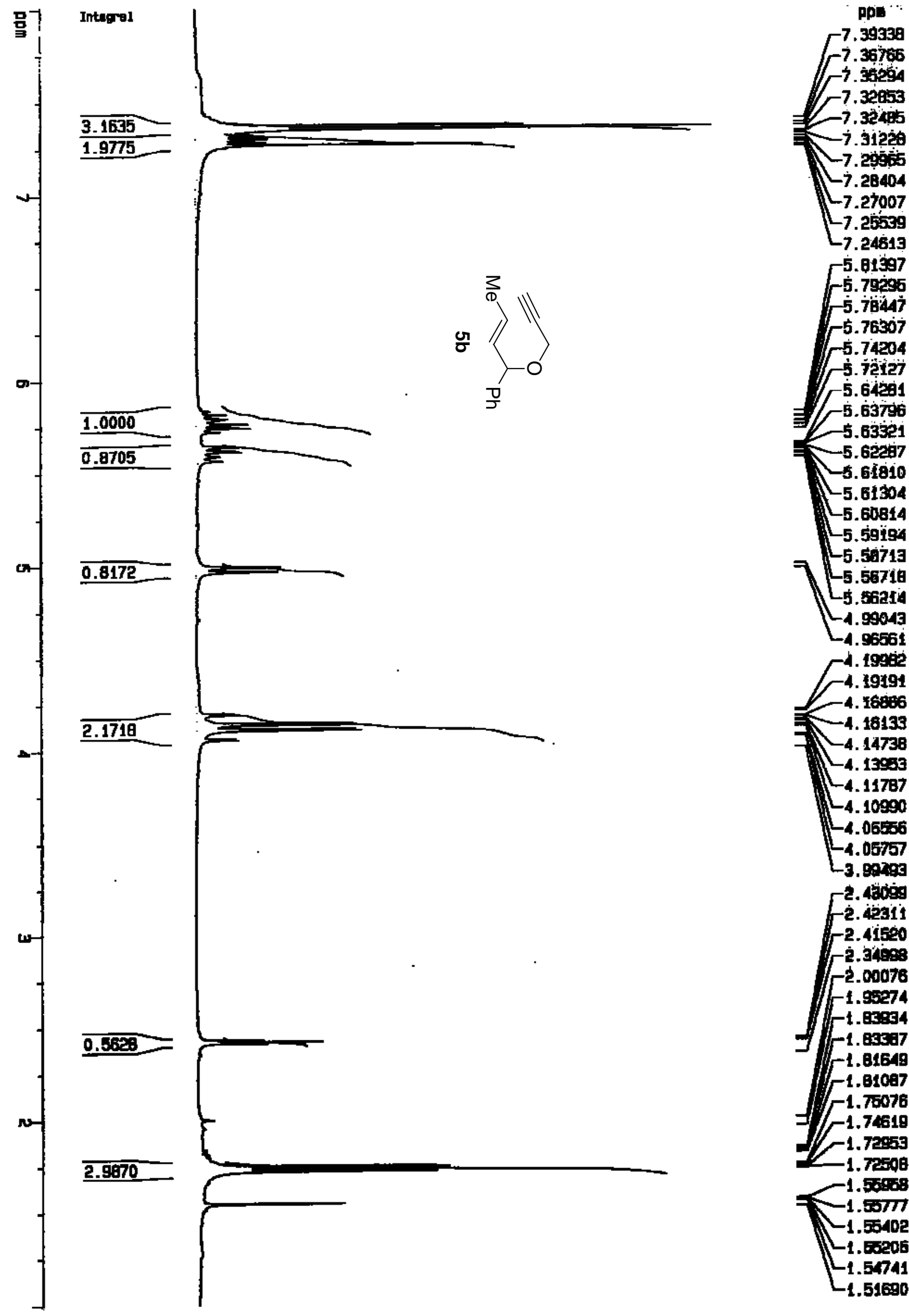

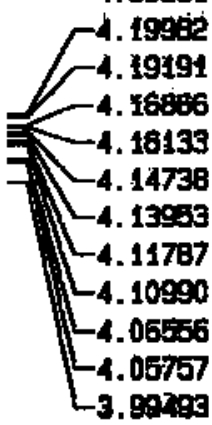

- 2. 4 tog

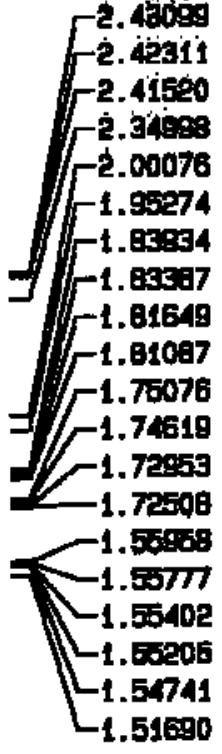




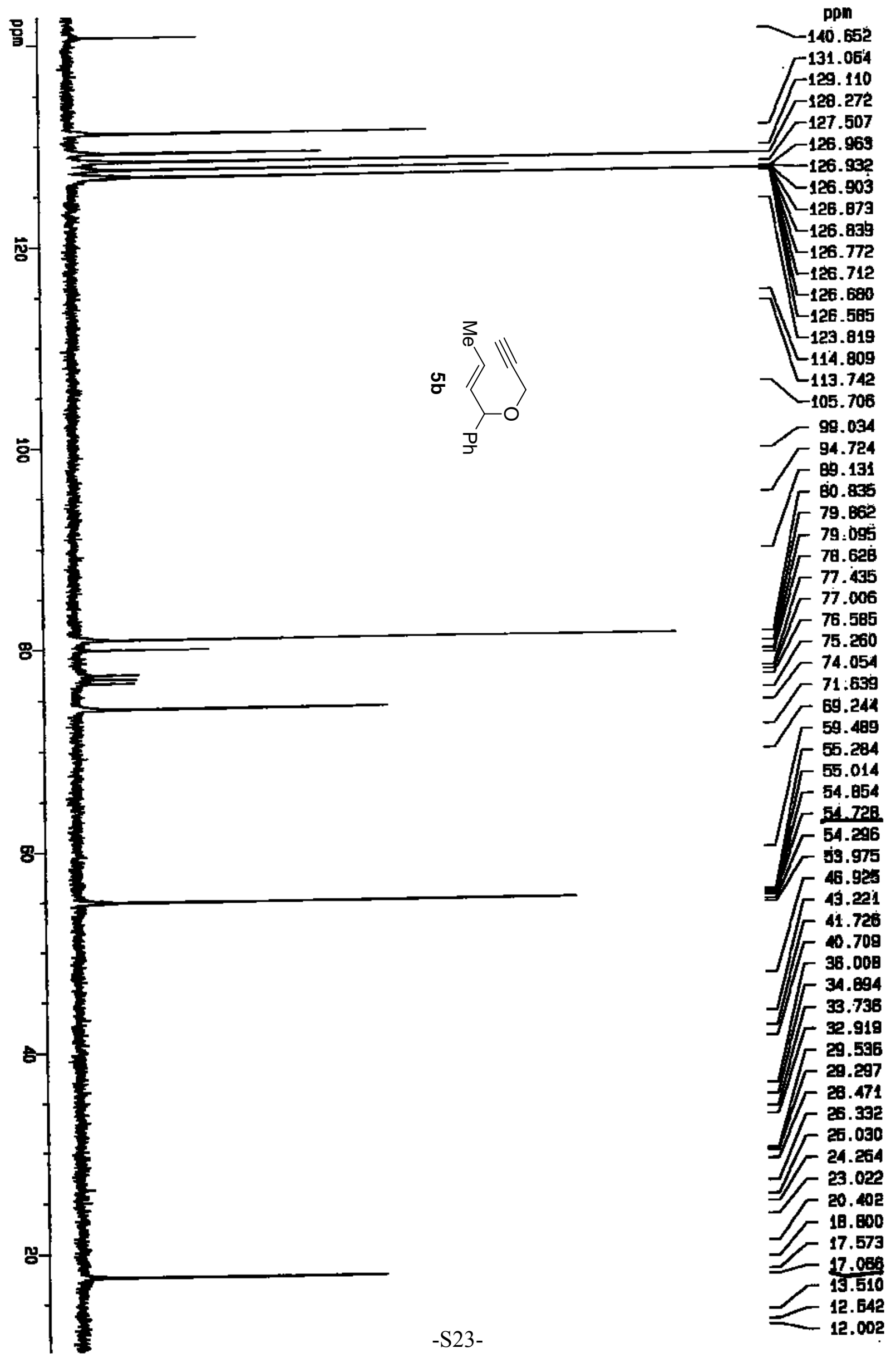




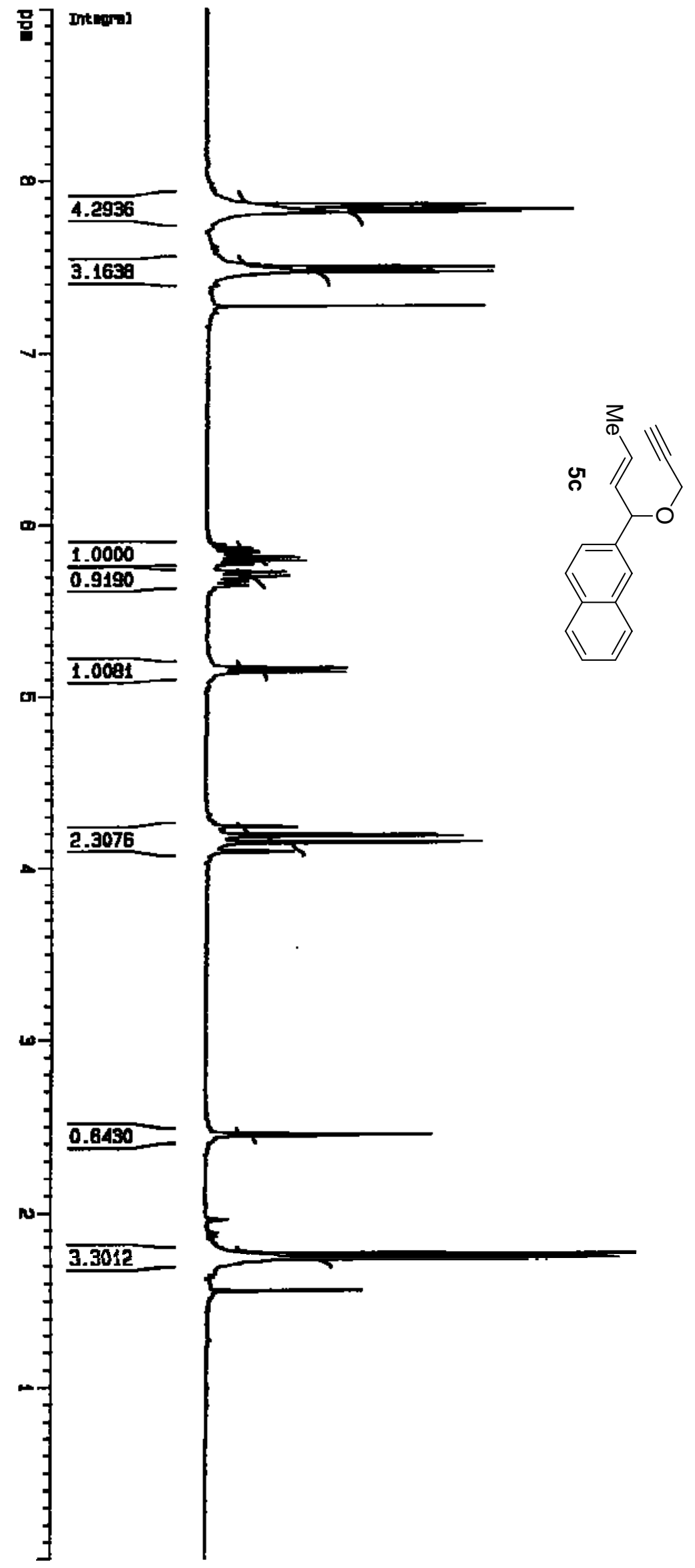

ppn

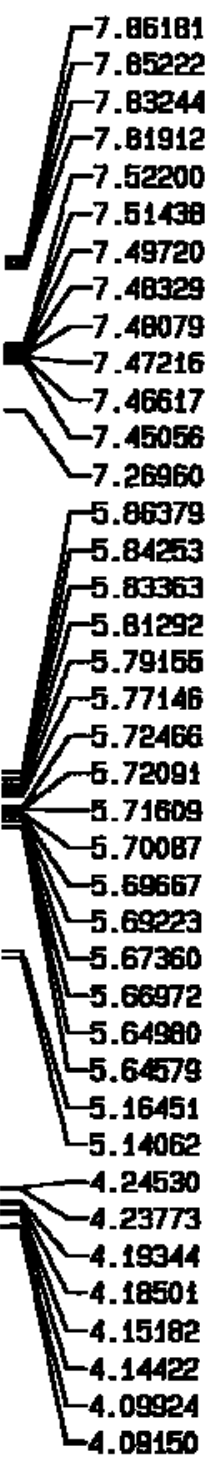



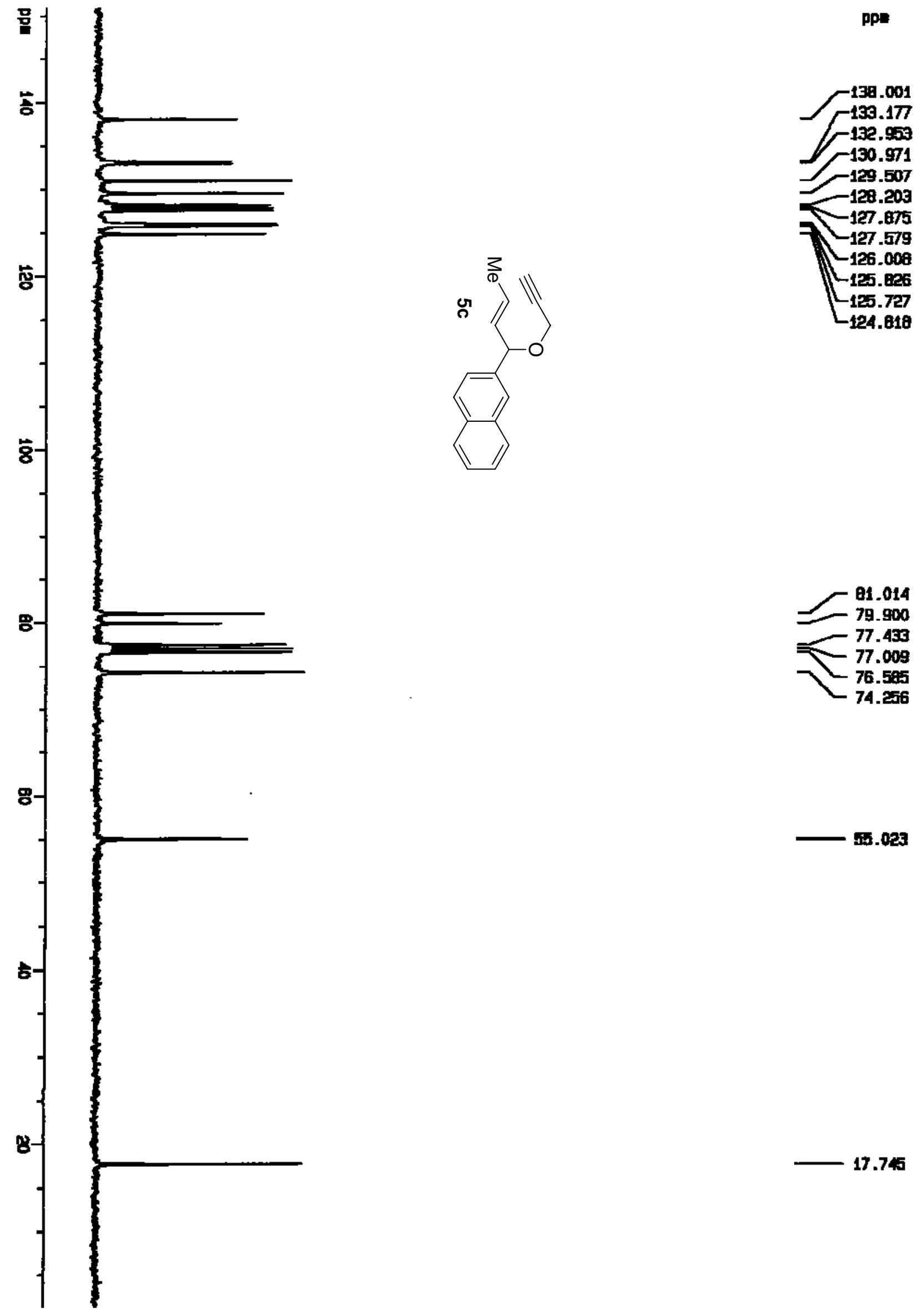

50.023 


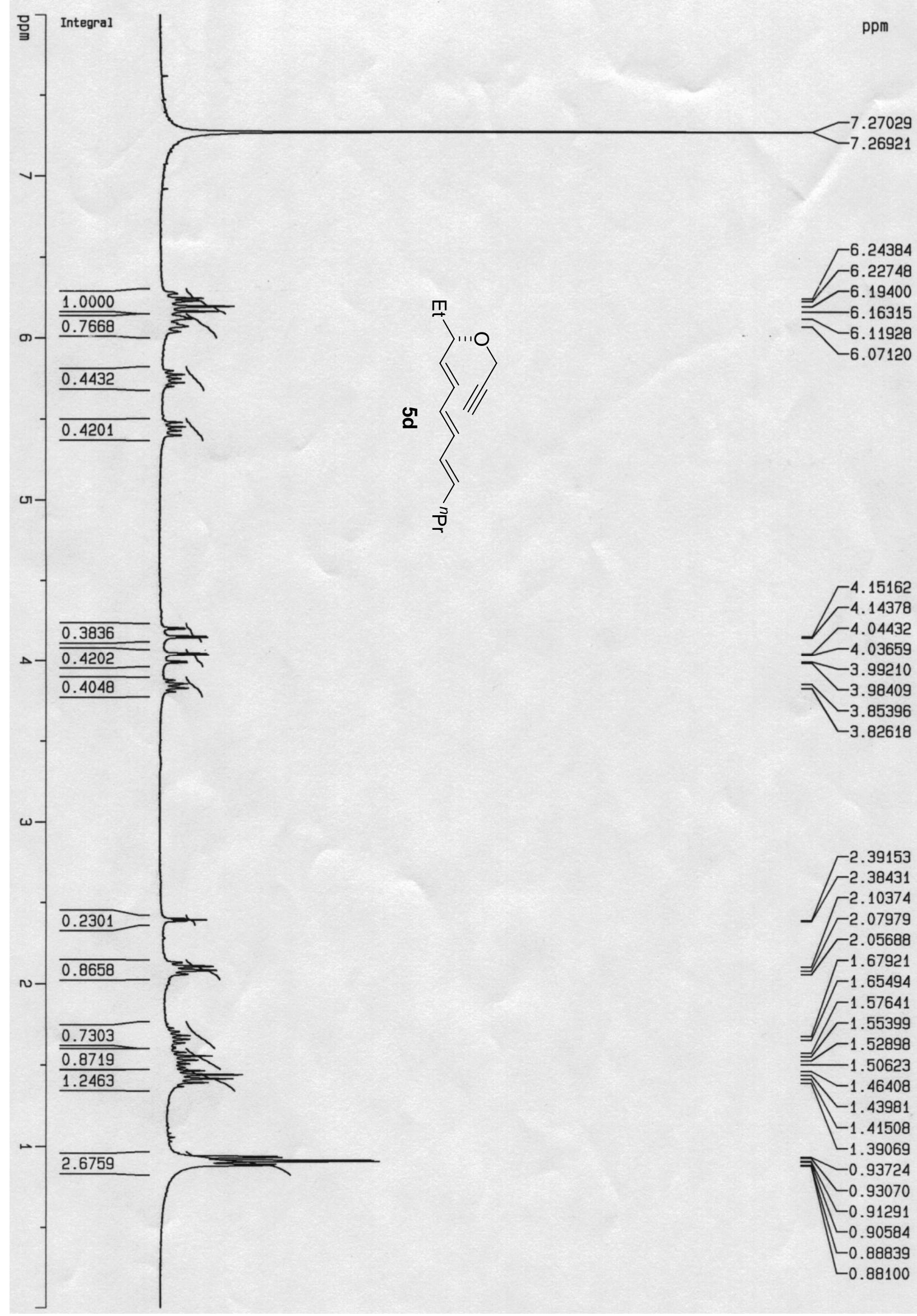




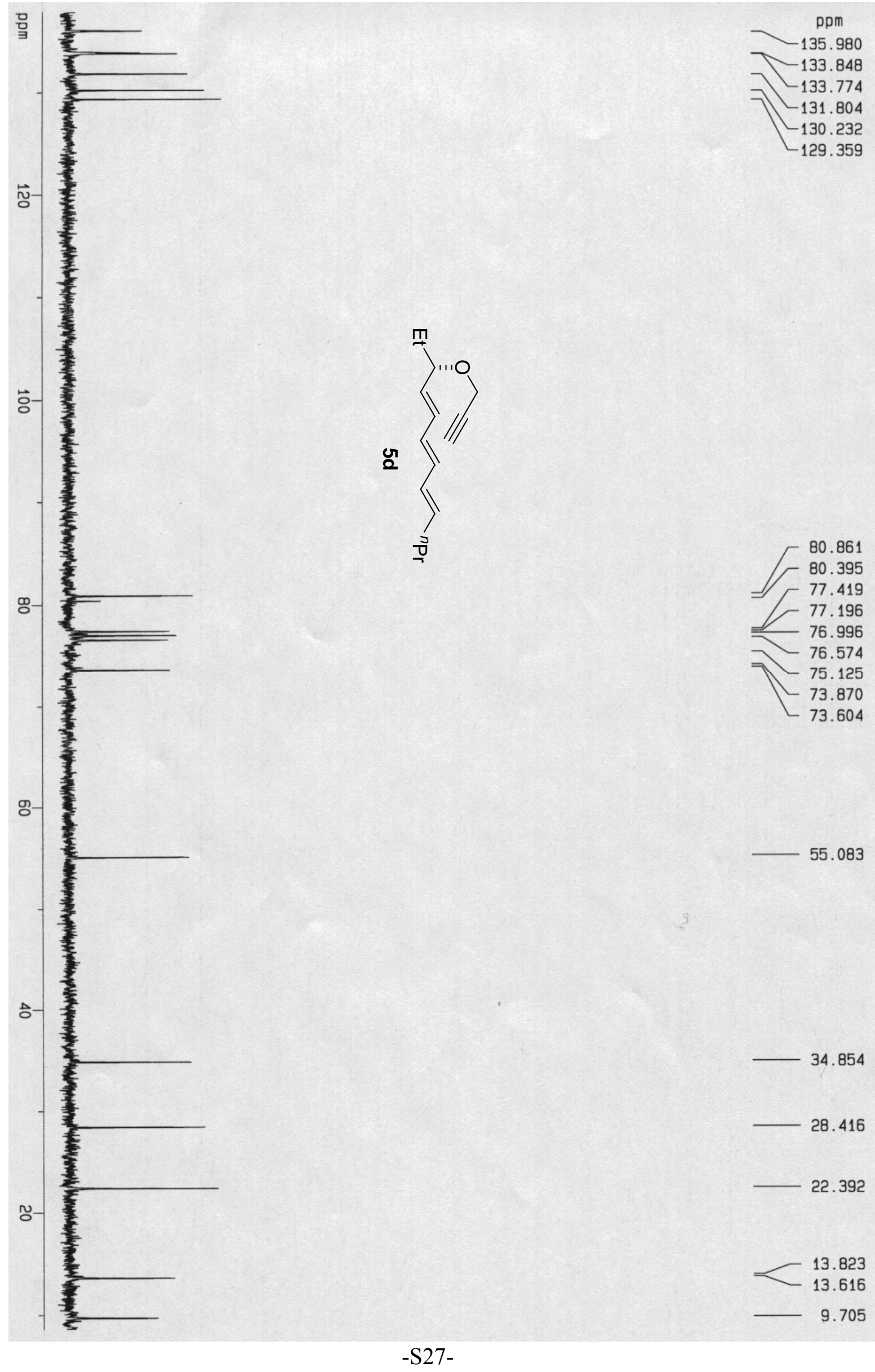




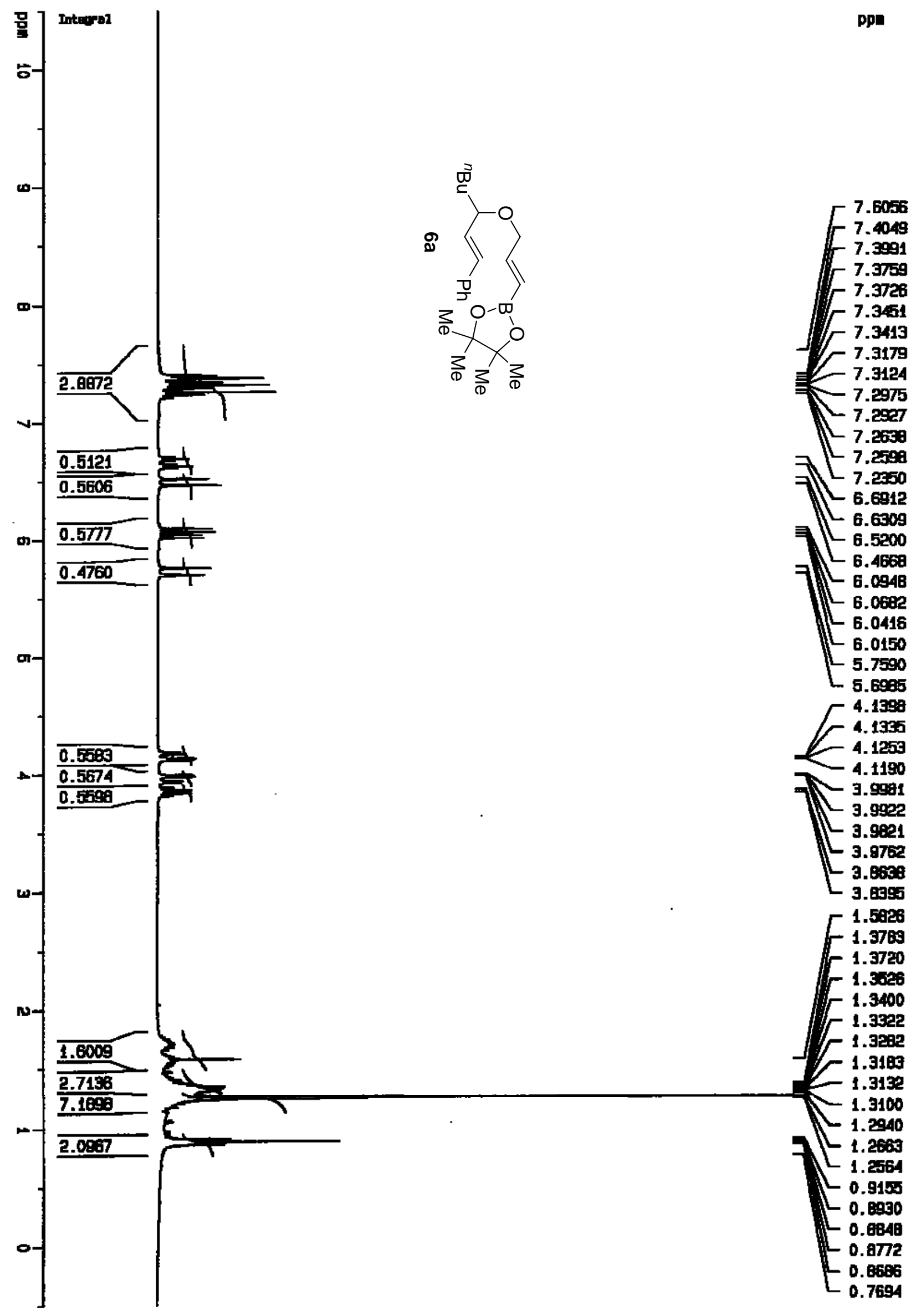




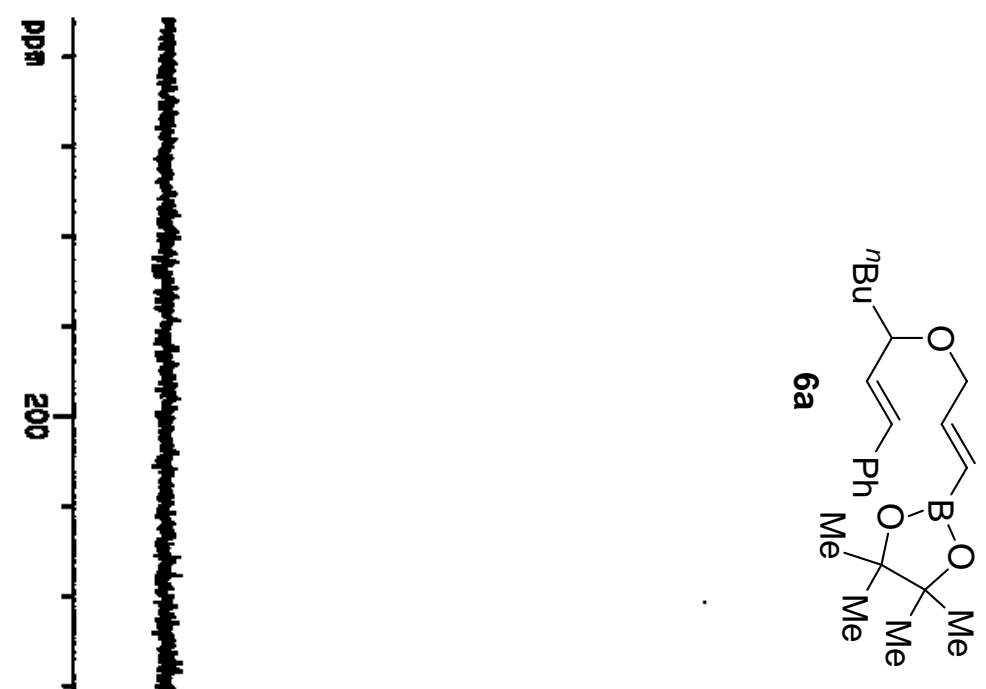

容-

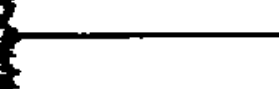

$-149.779$
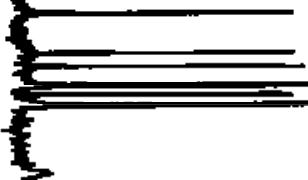

5

8
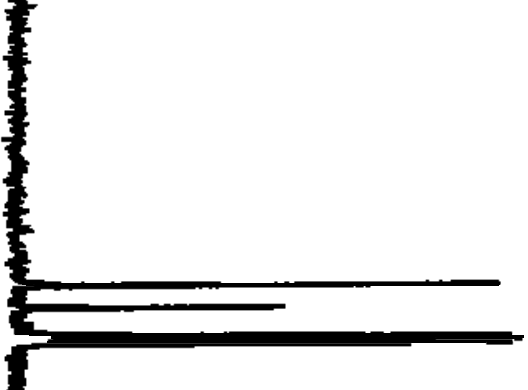

.030

80.441

77.427

77.003

76.579

69.517

8

$3-$

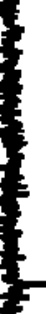

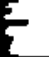

27.410

24.640

22.609

13.969 


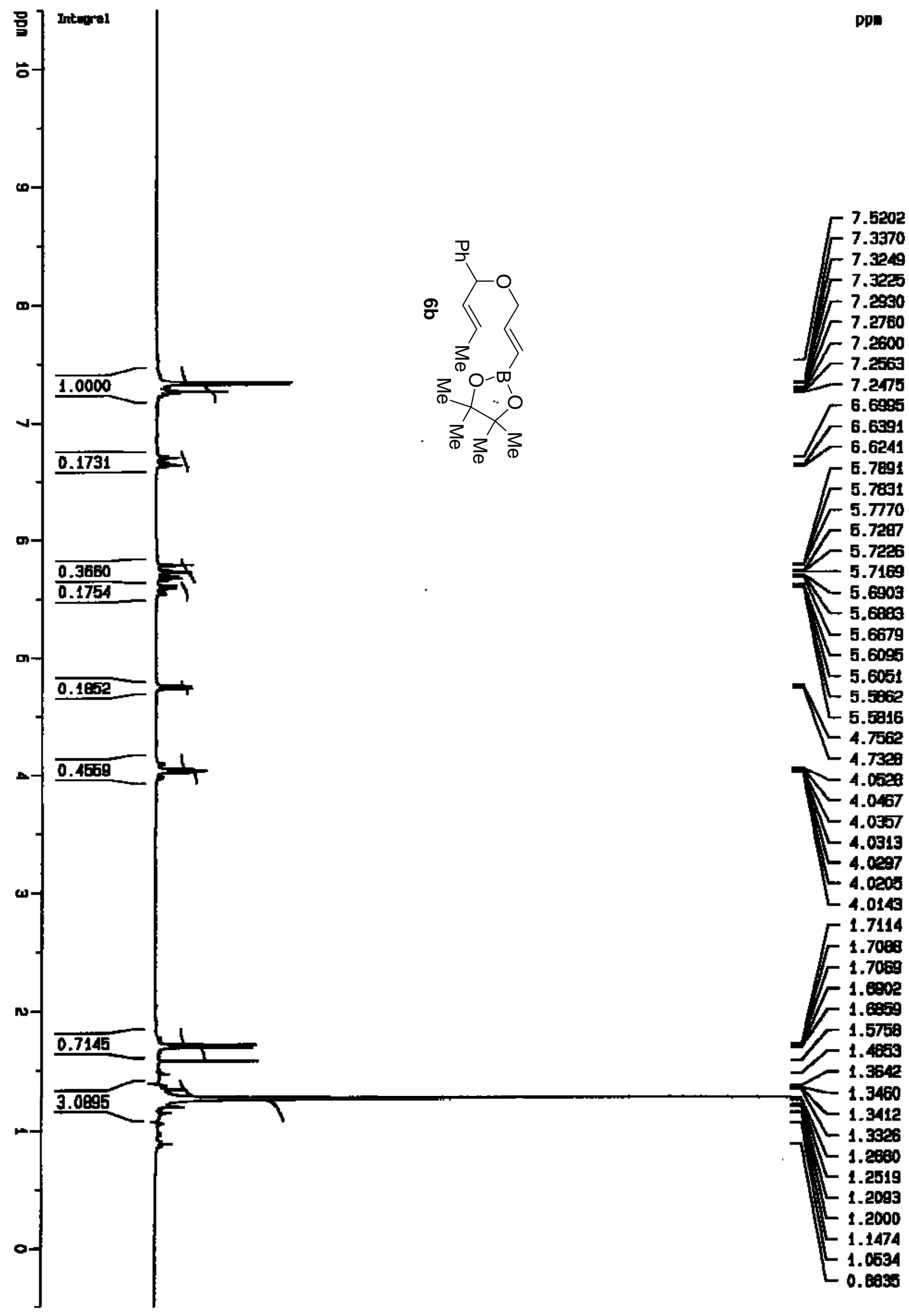


Nelson, S. G., Bungard, C. J. and Stevens, B. D.

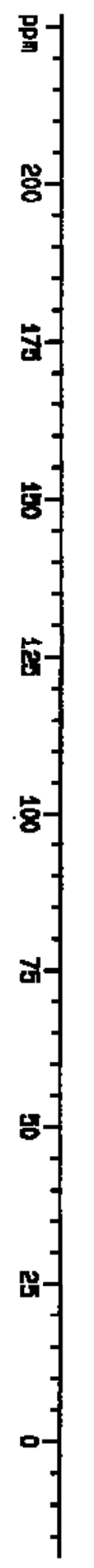

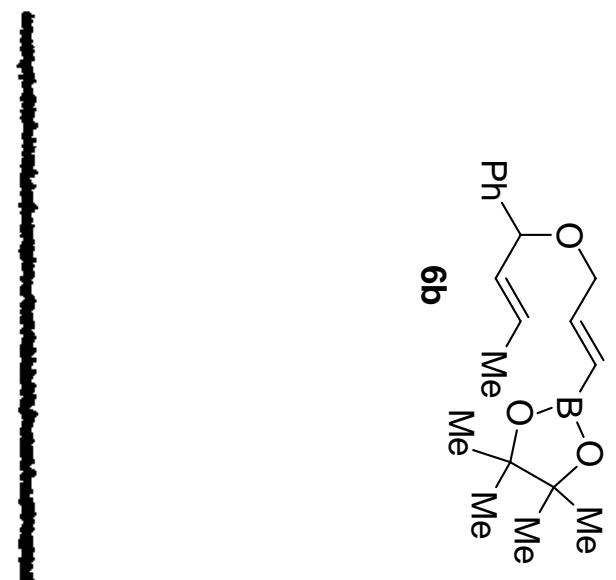

Ppn
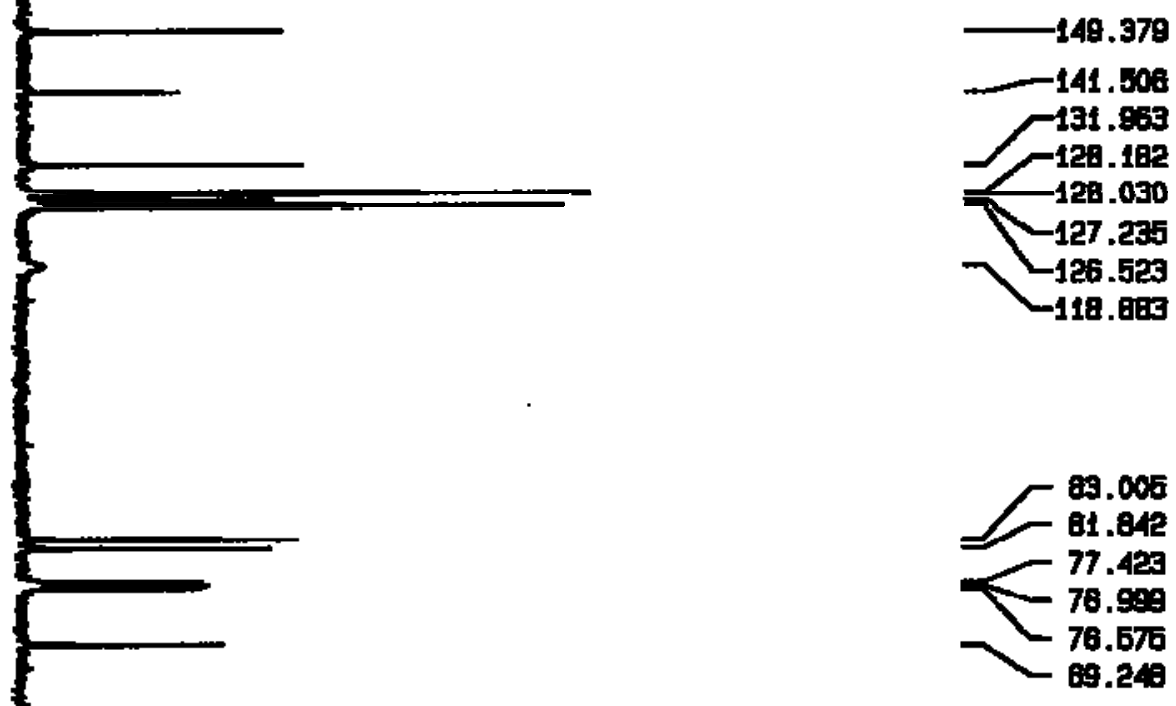

31.435

24.601

20.498
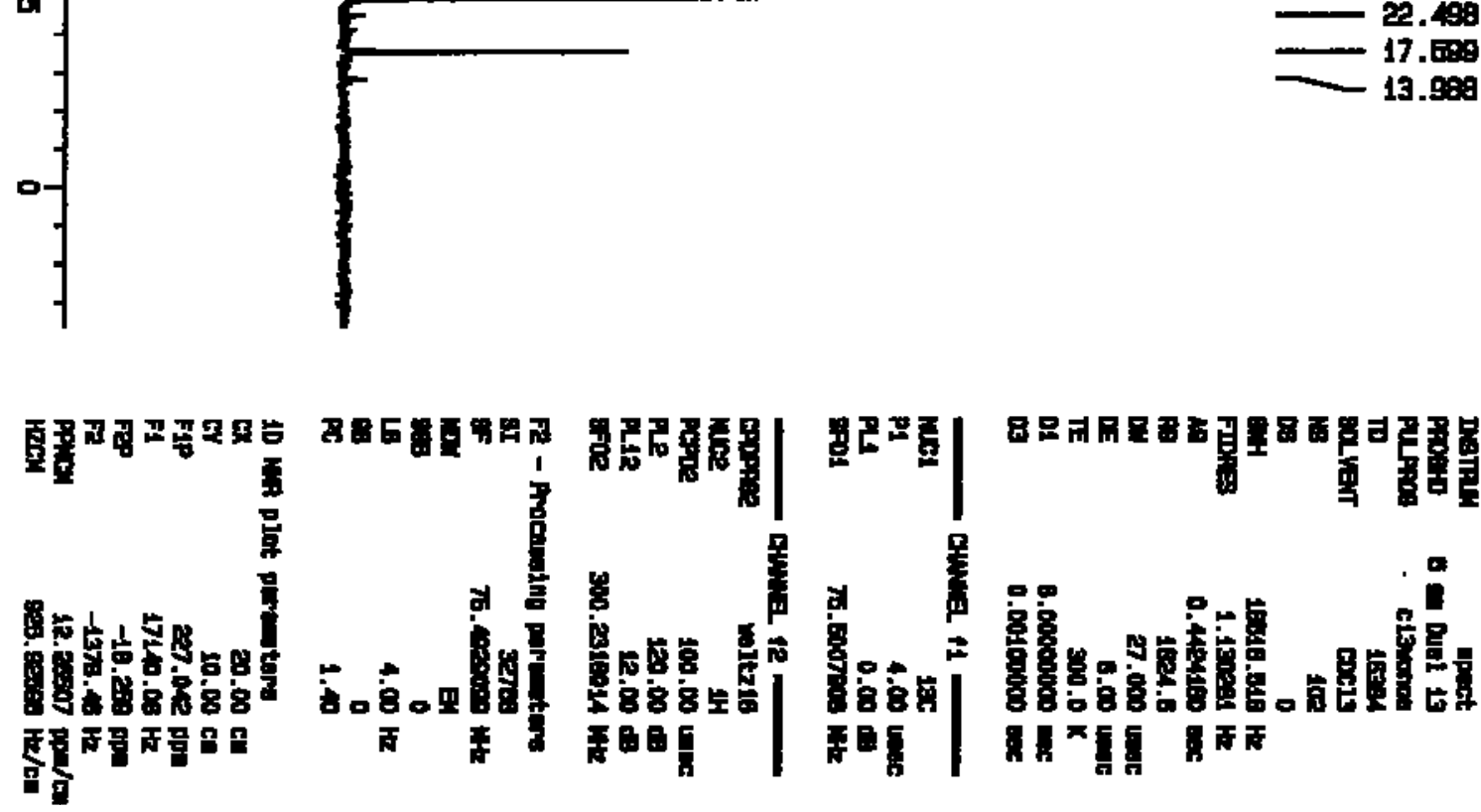

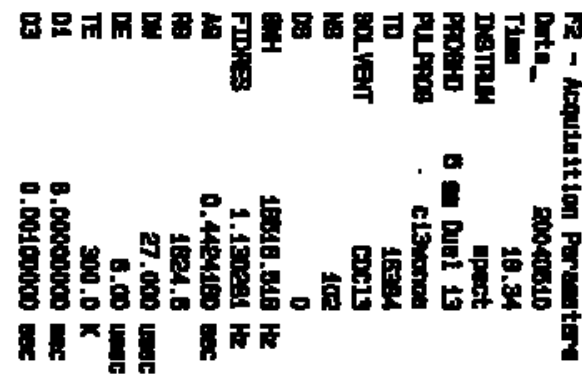

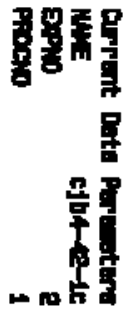



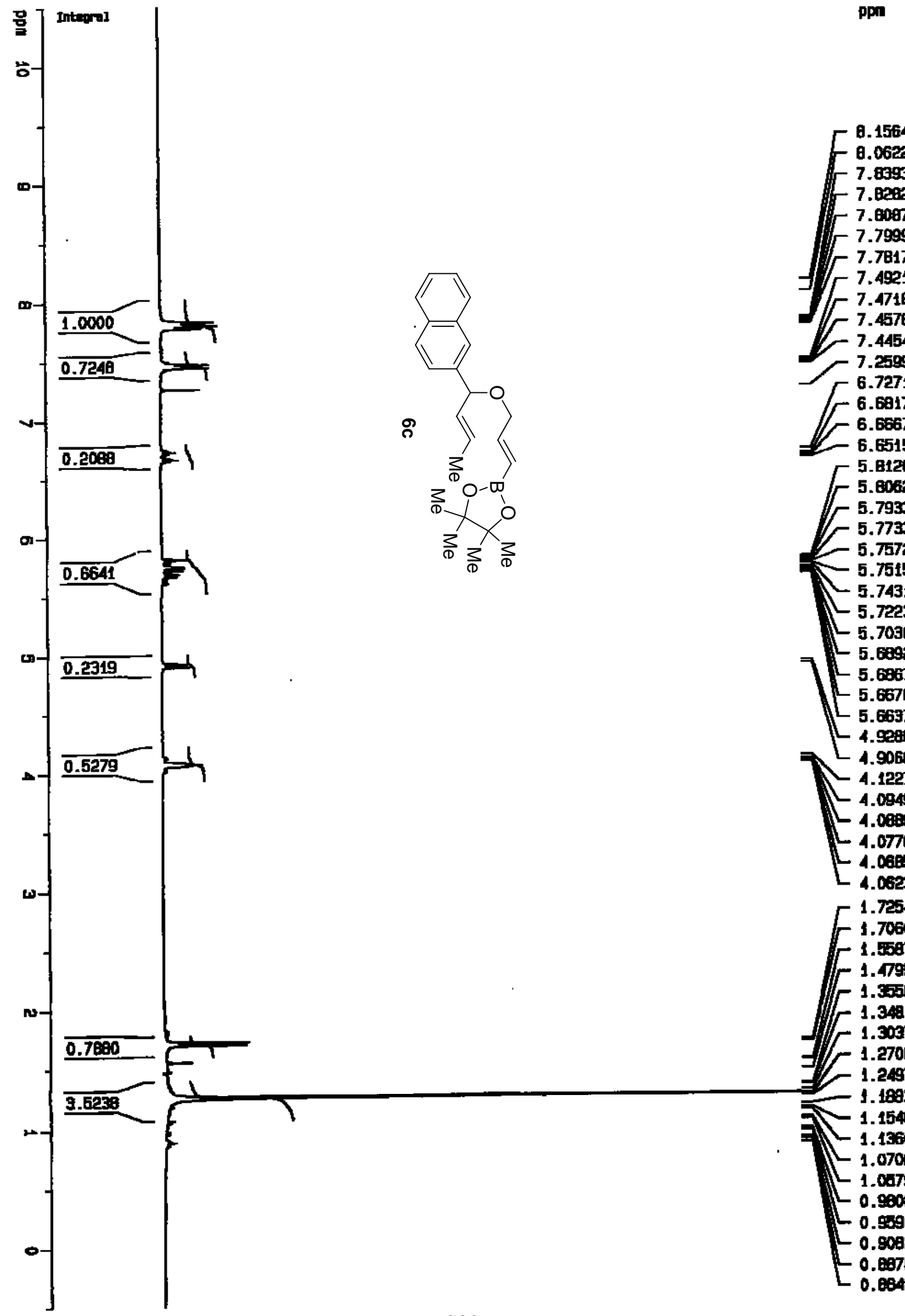

7.8999

$-7.0282$

[ 7.6007

- 7.7817

7.4921

7.4718

7.4578

$-7.4454$

7.2590

6.7271

- 6.6917

$-6.6667$

$-6.6515$

5.8120

5.8062

5.7930

$-5.7739$

5.7572

$-5.7515$

$-5.7431$

$-5.7223$

$-5.7036$

75.6092

- 5.6867

5.6676

$-5.6697$

$-4.9286$

4.9066

$-4.1227$

$-4.0949$

$-4.0089$

4.0778

$-4.0685$

$-4.0629$

$-1.7254$

$-1.7060$

$-1.5087$

$-1.4795$

$r^{1.3558}$

$-1.3481$

1.3037

1.2708

1.2497

1.1893

7) 1.1546

$=1.1350$

$-1.0706$

$-1.0679$

0.9608

$-0.9590$

L 0.9092

0.8076

0.6842 


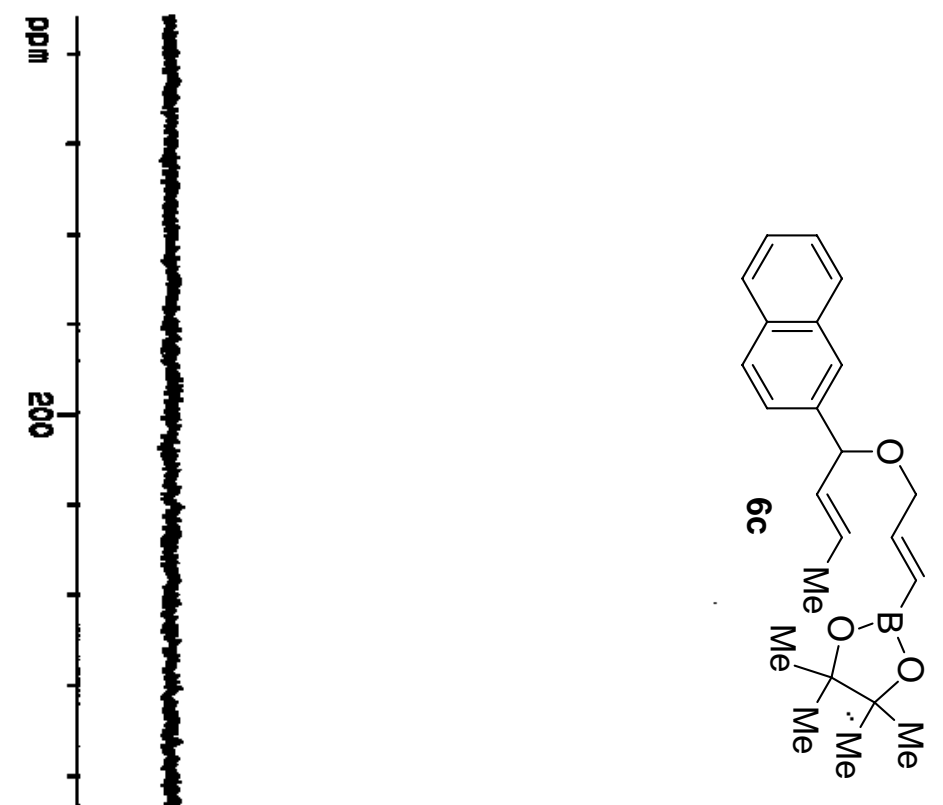

Dp:
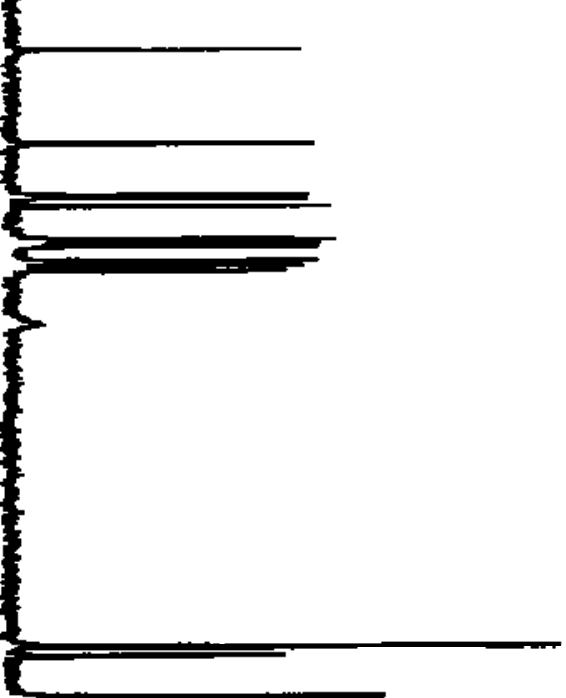

형-
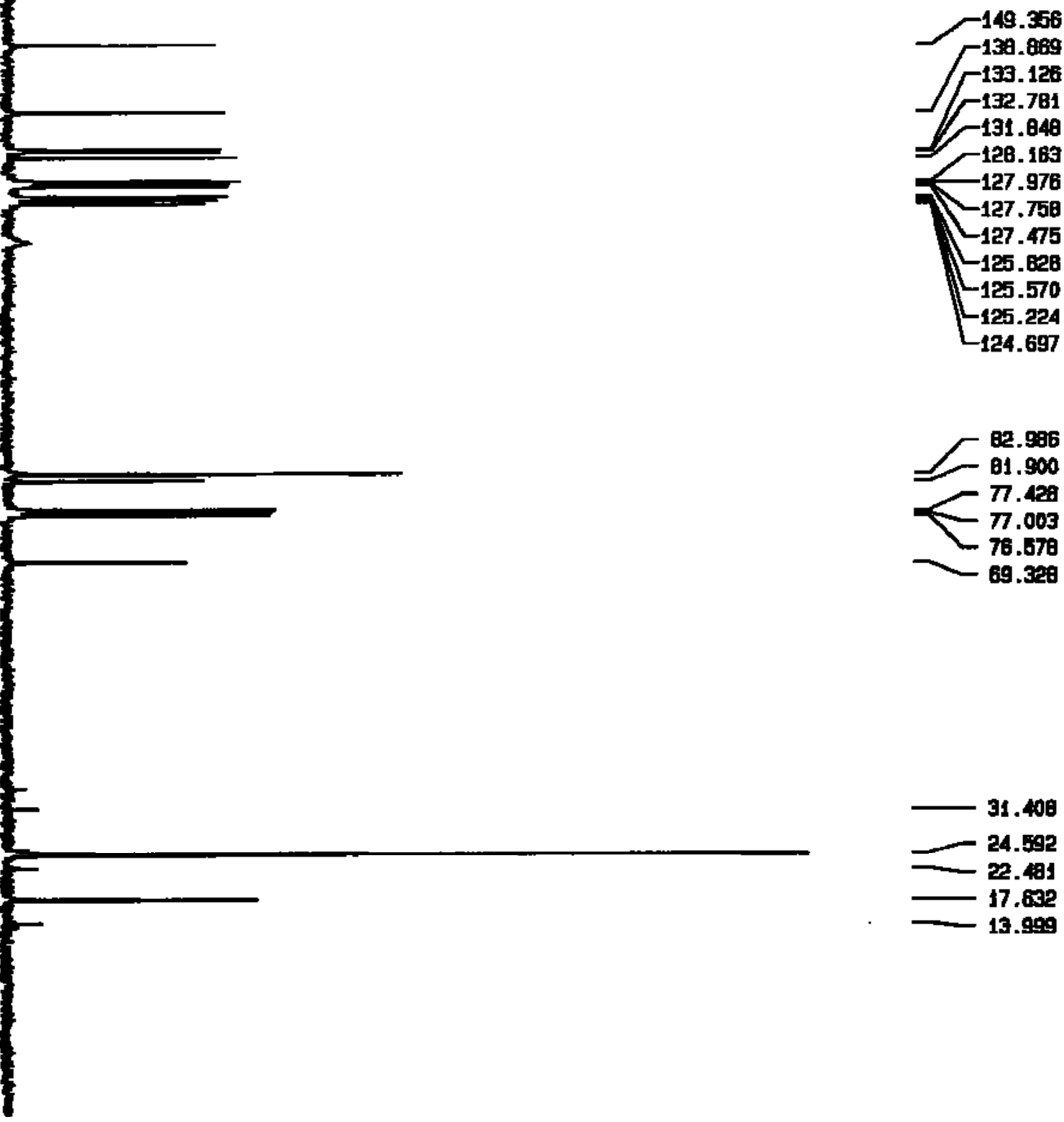

官-

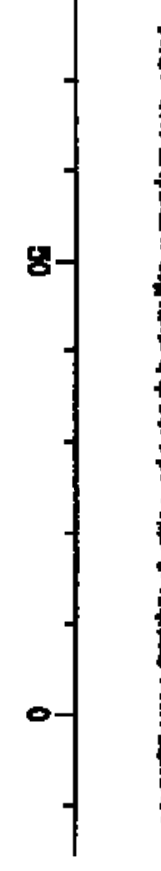



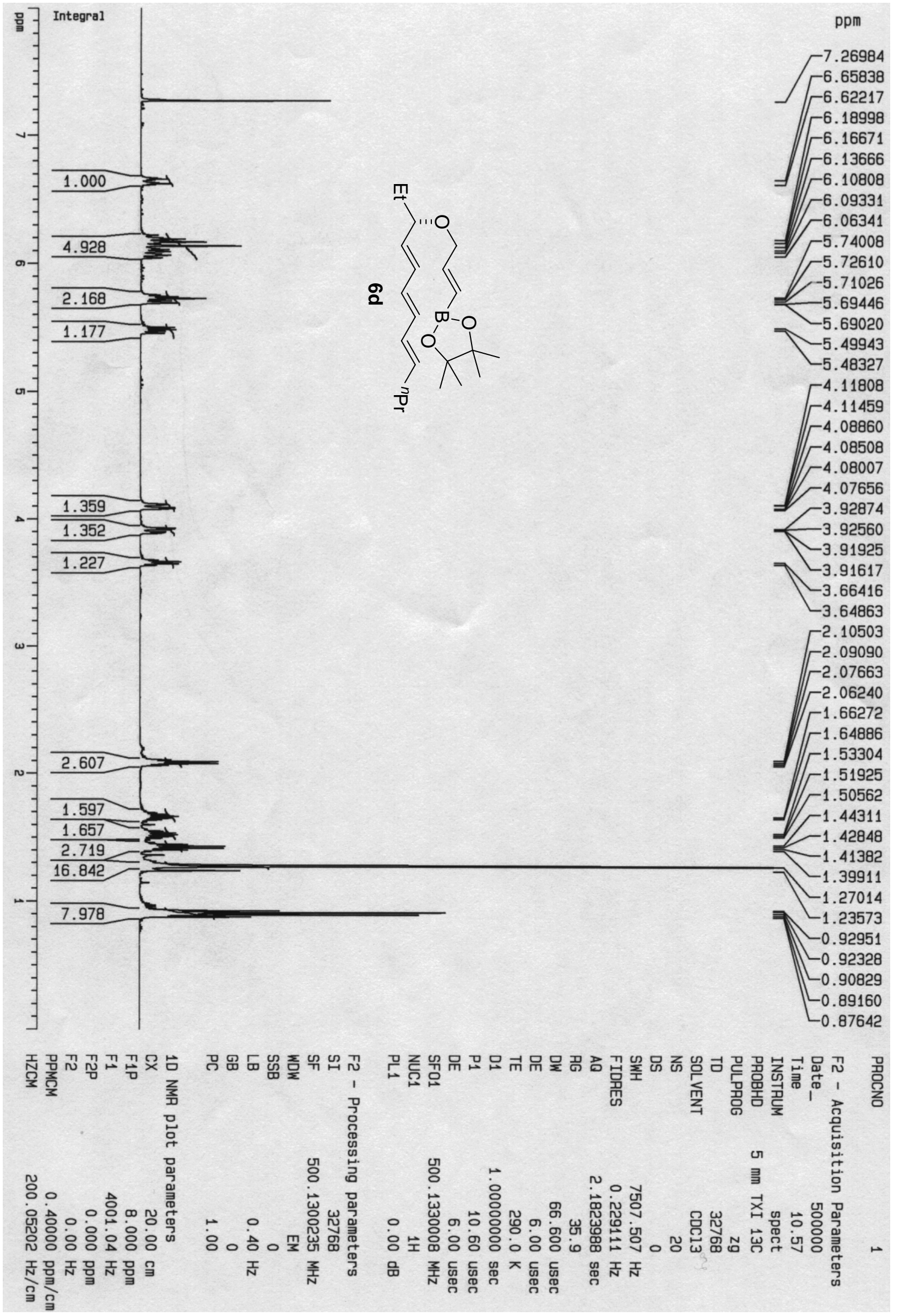


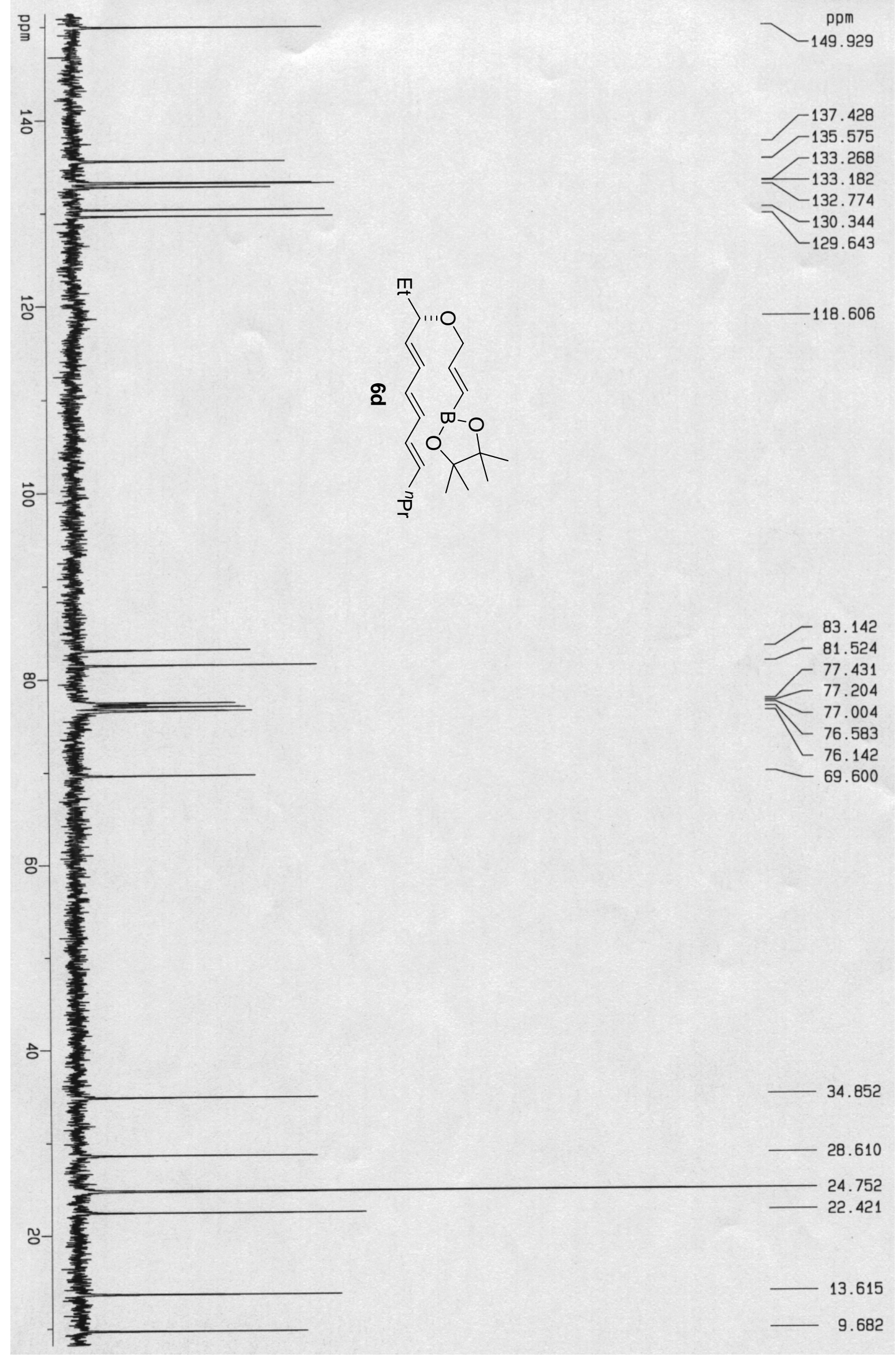



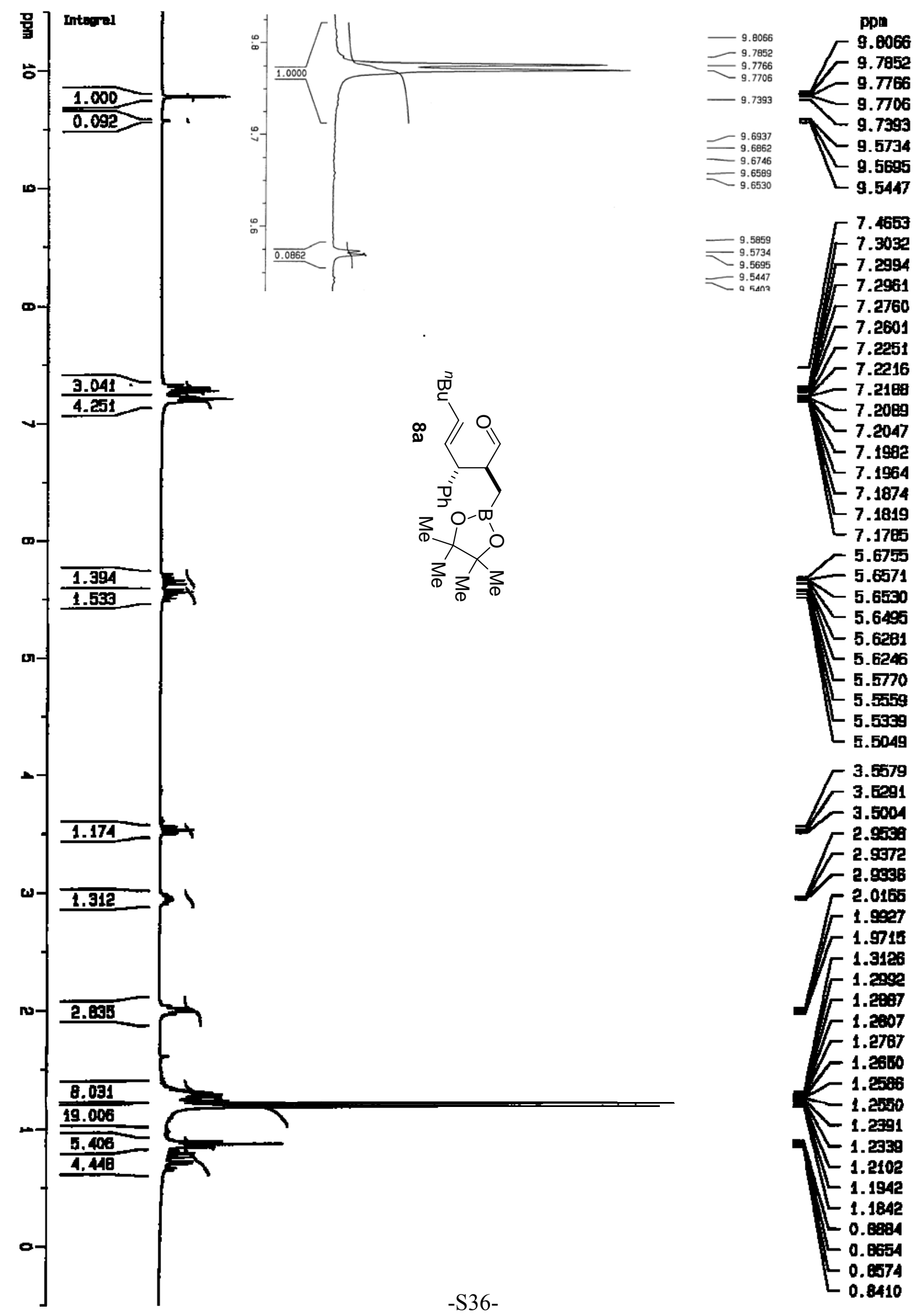

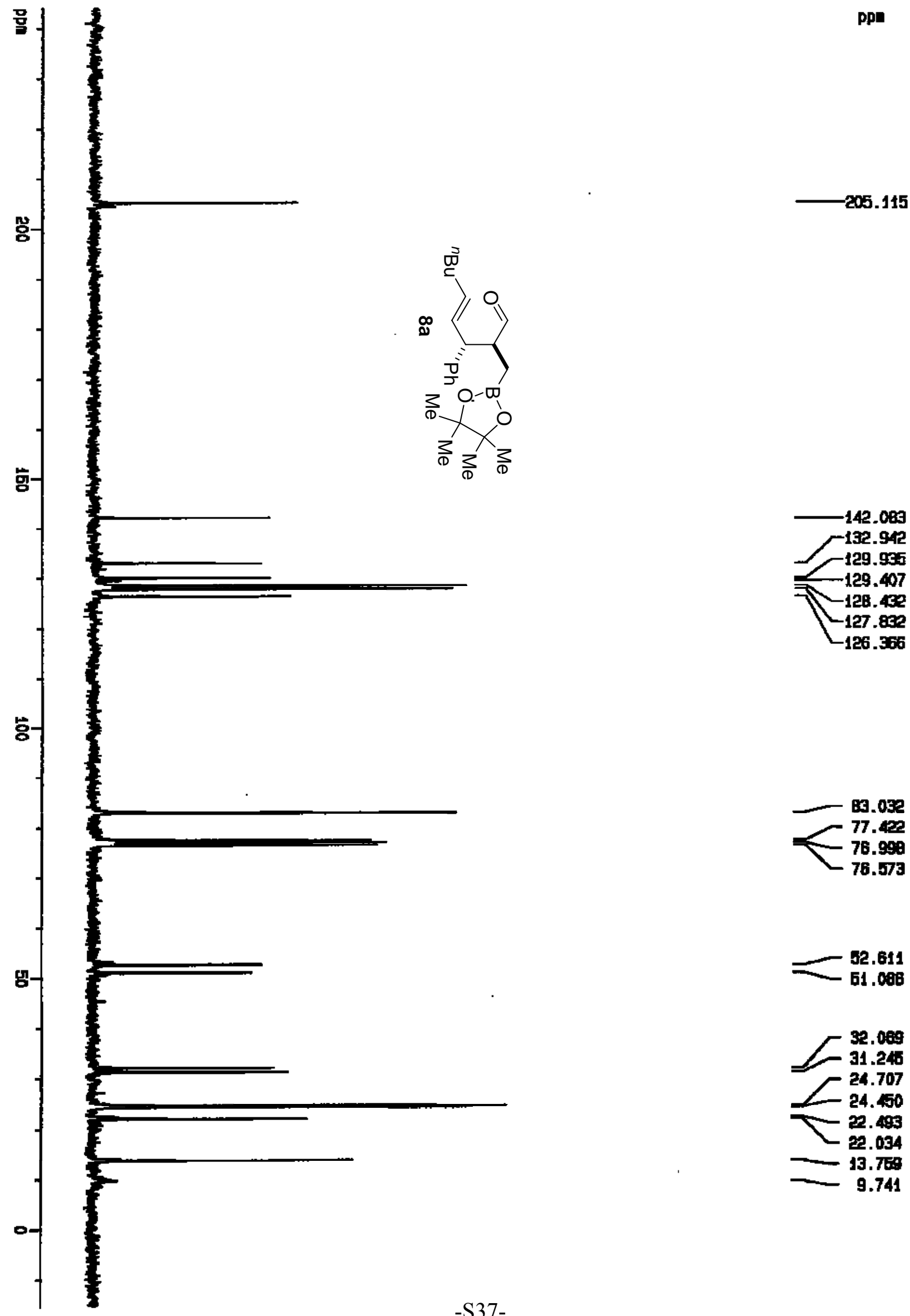


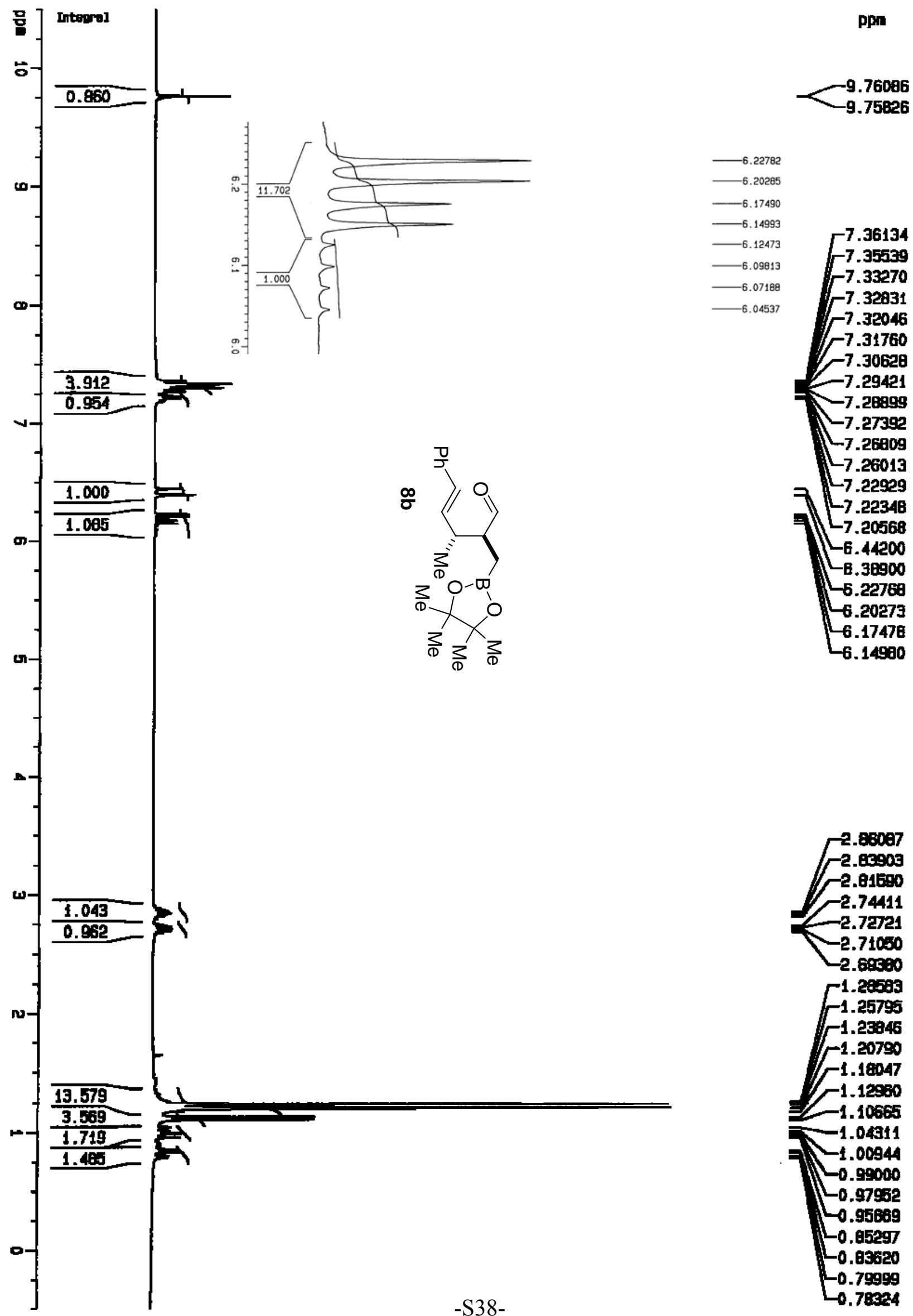



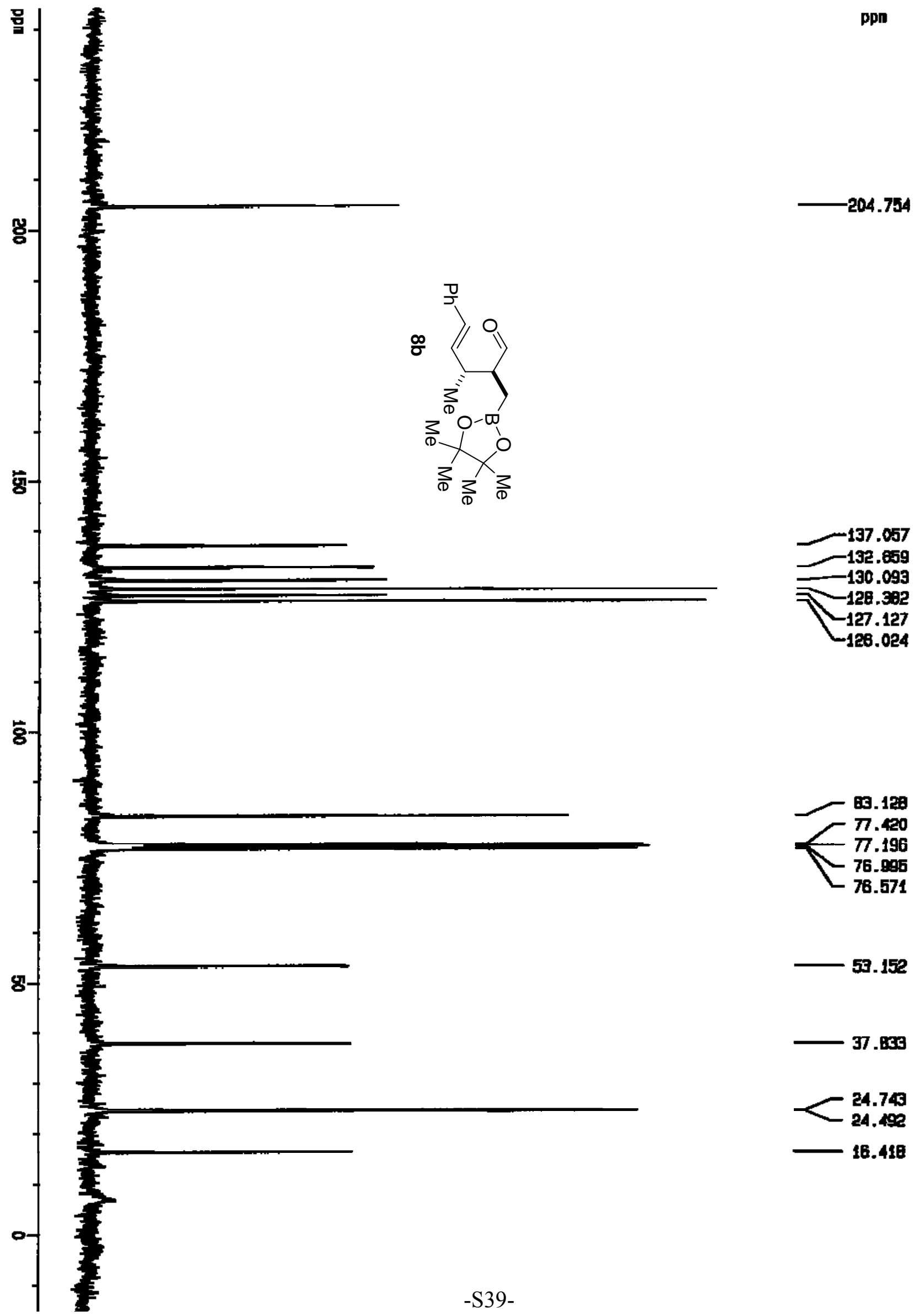

69.129

$\pi .420$

$\pi .196$

76.995

76.571

59.152

37.853

24.743

24.492

16.418 


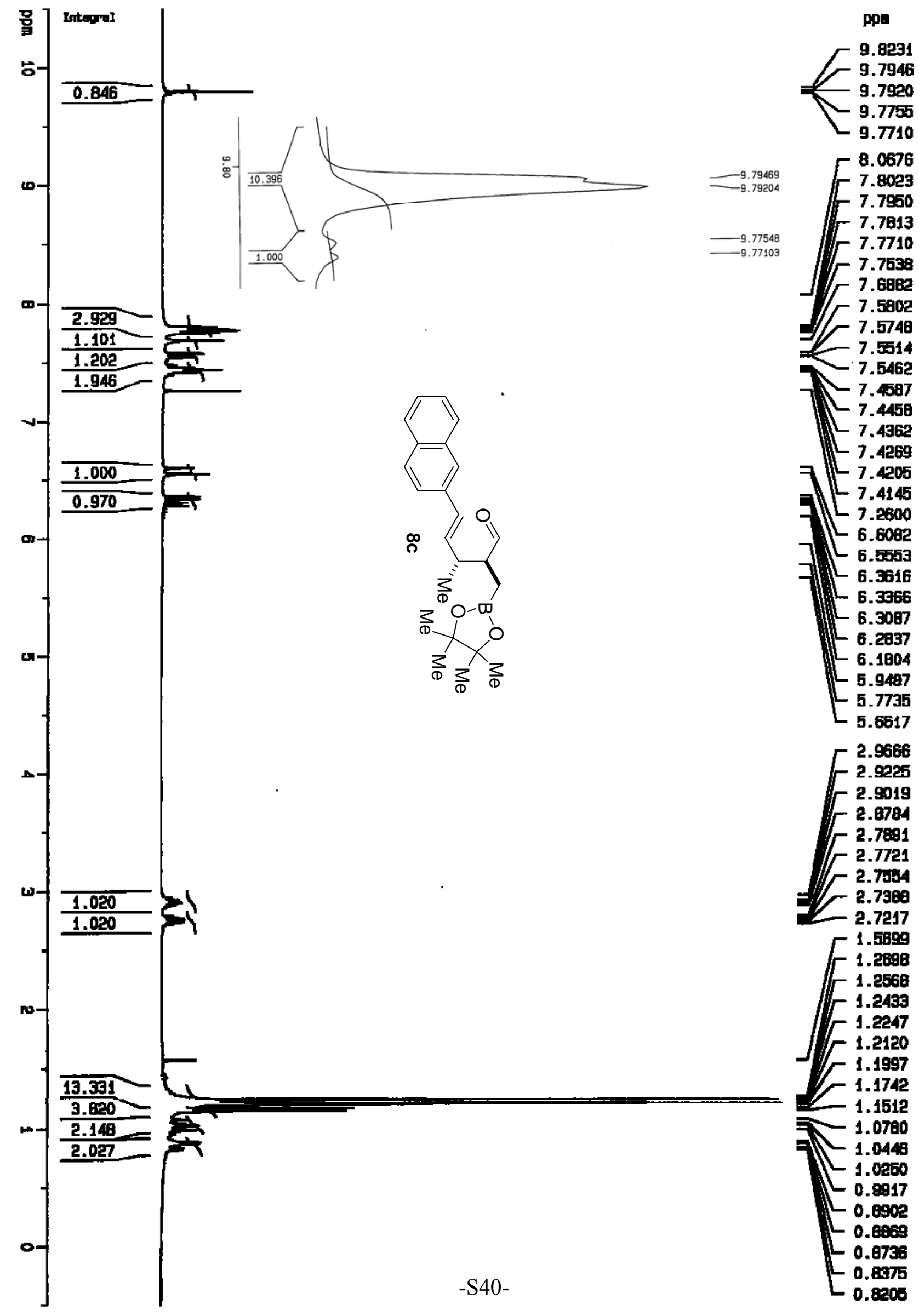




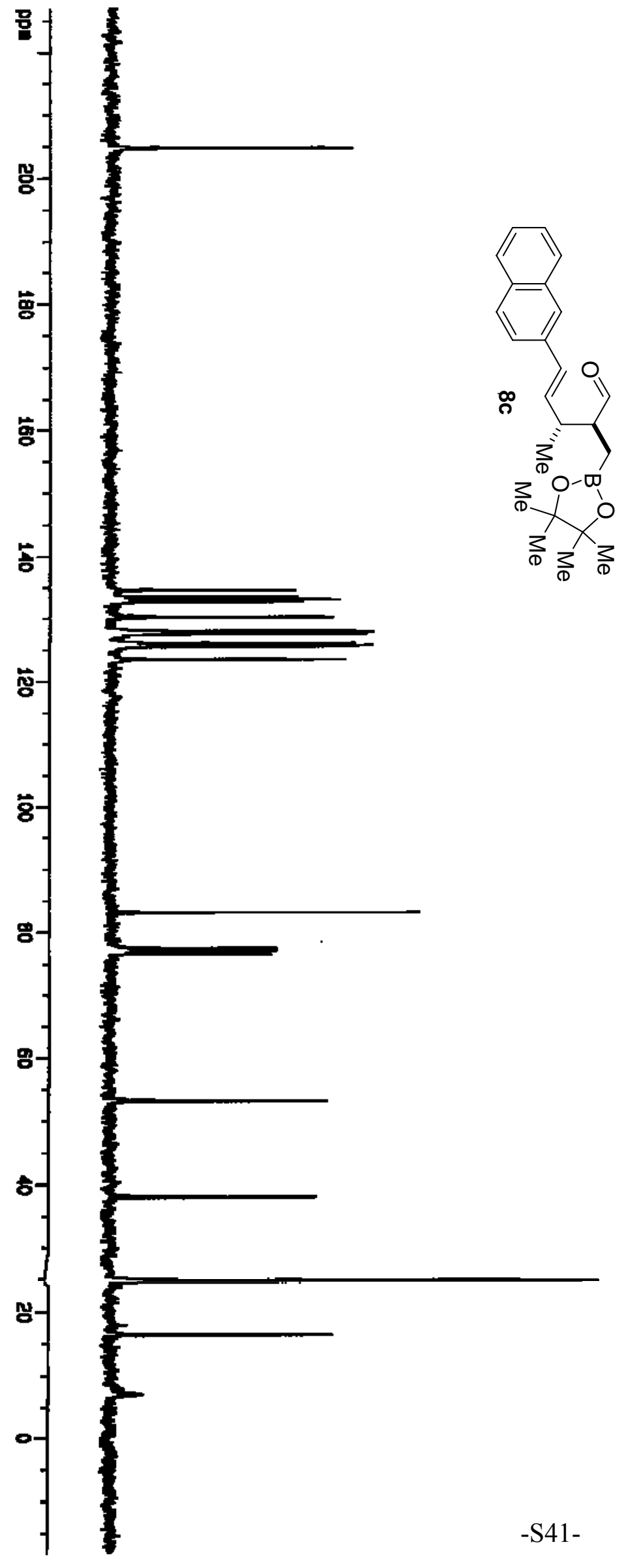

pola

$-204.678$

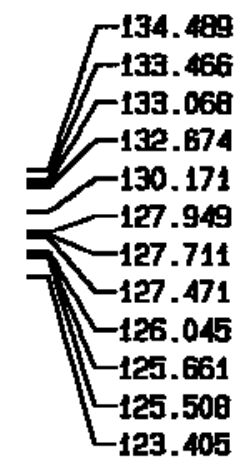

89.107

77.427

77.002

76.578

55.155

37.906

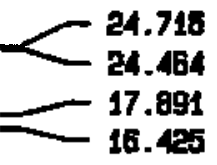

6.913 


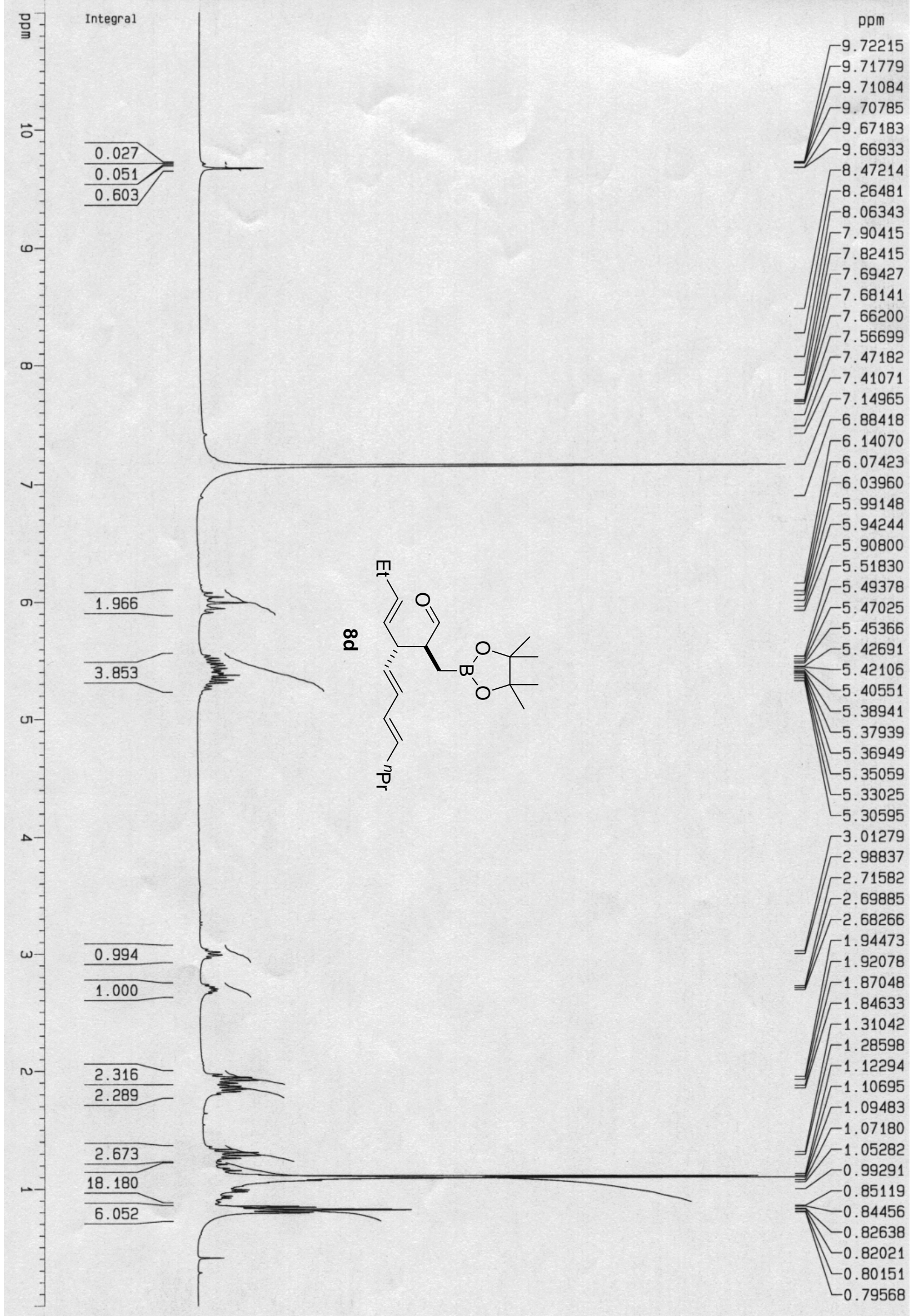




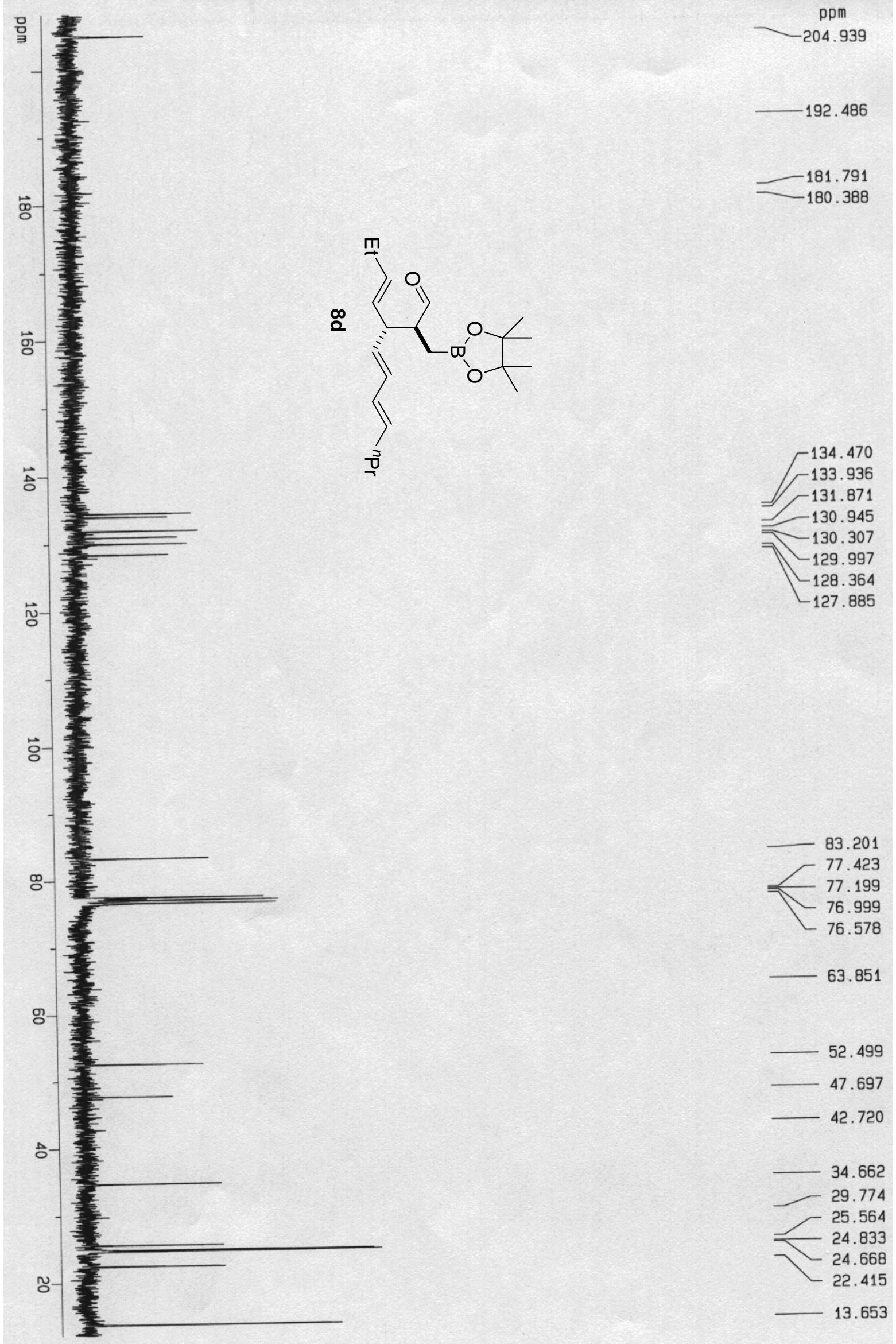




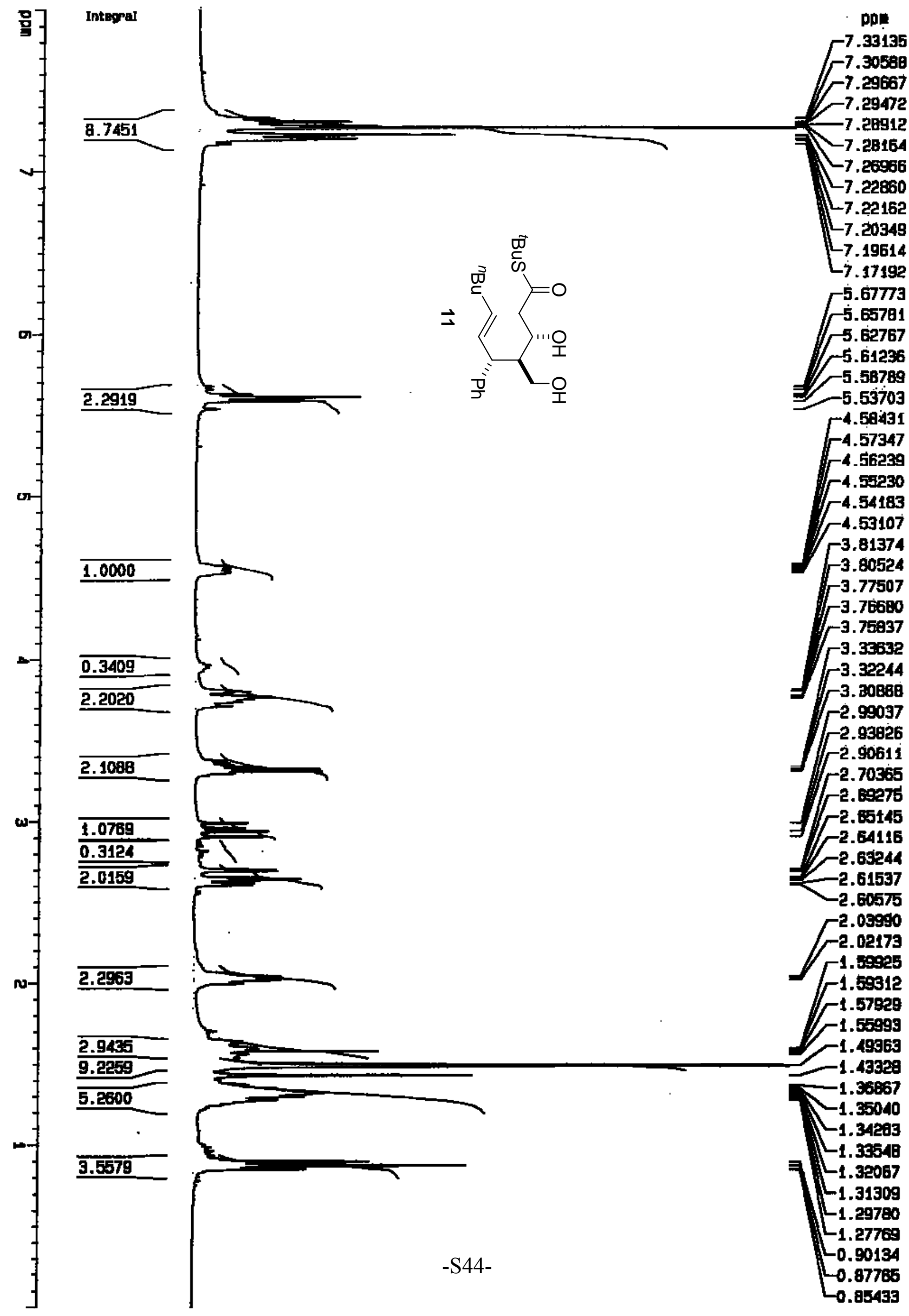



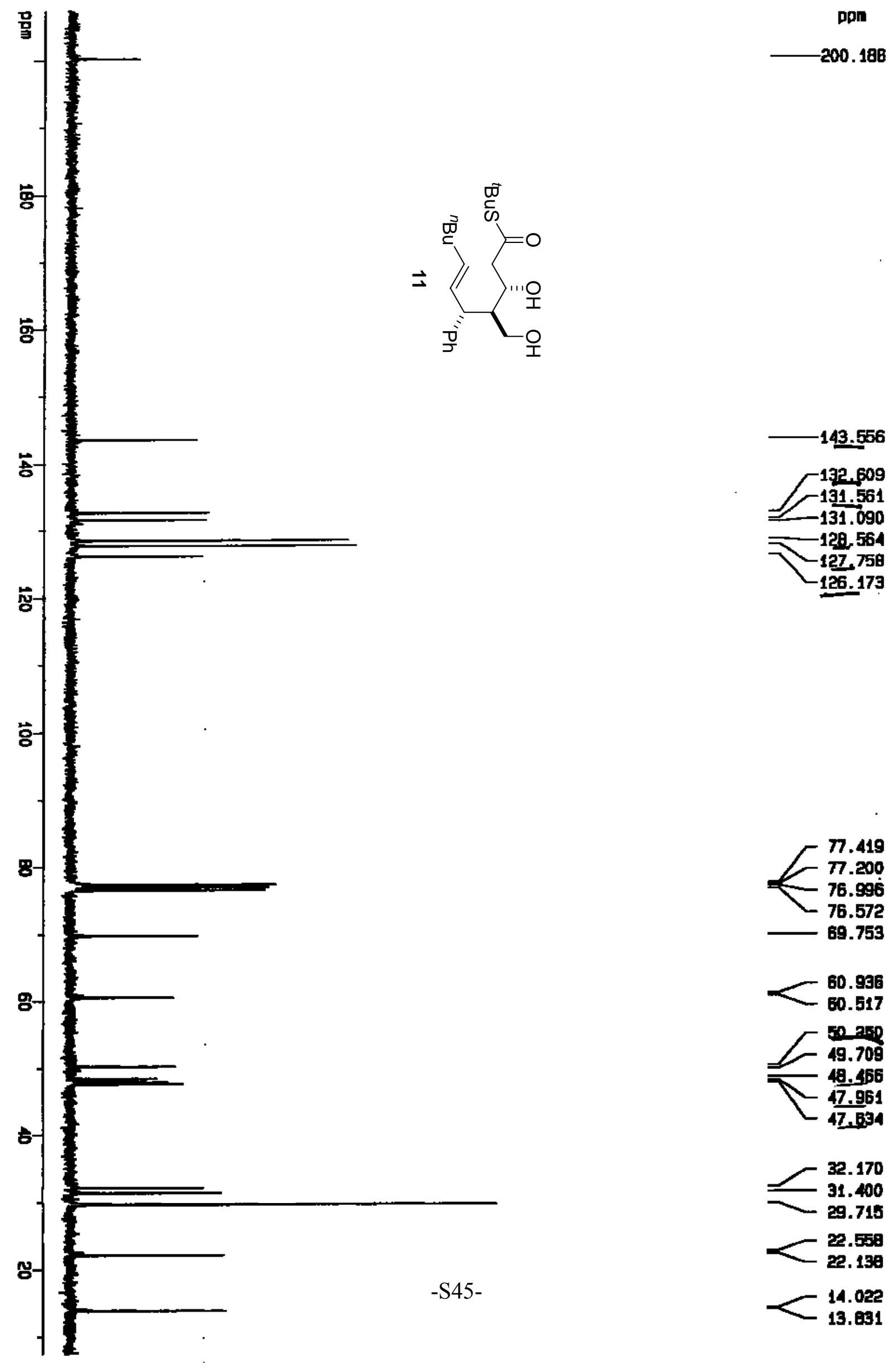


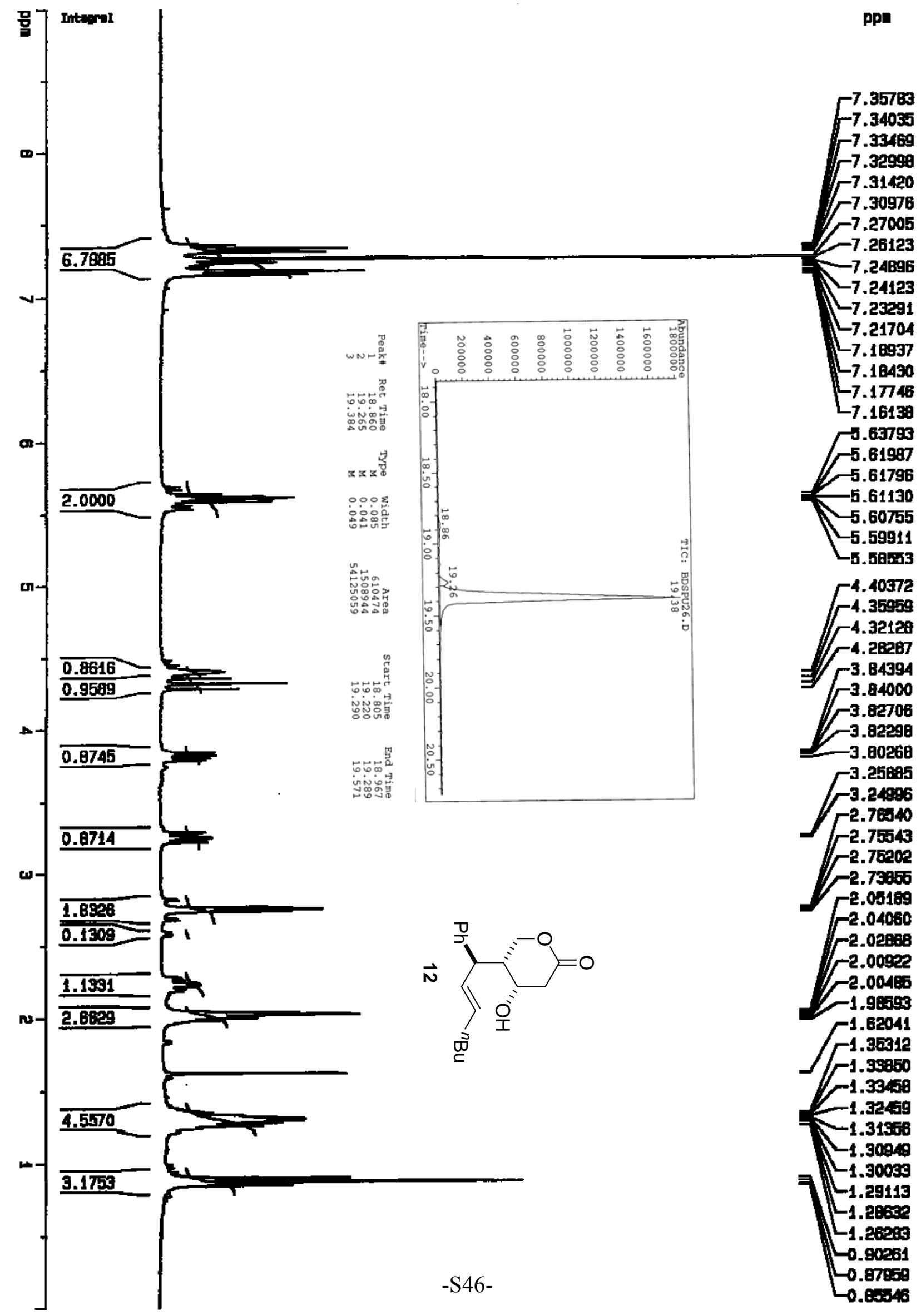




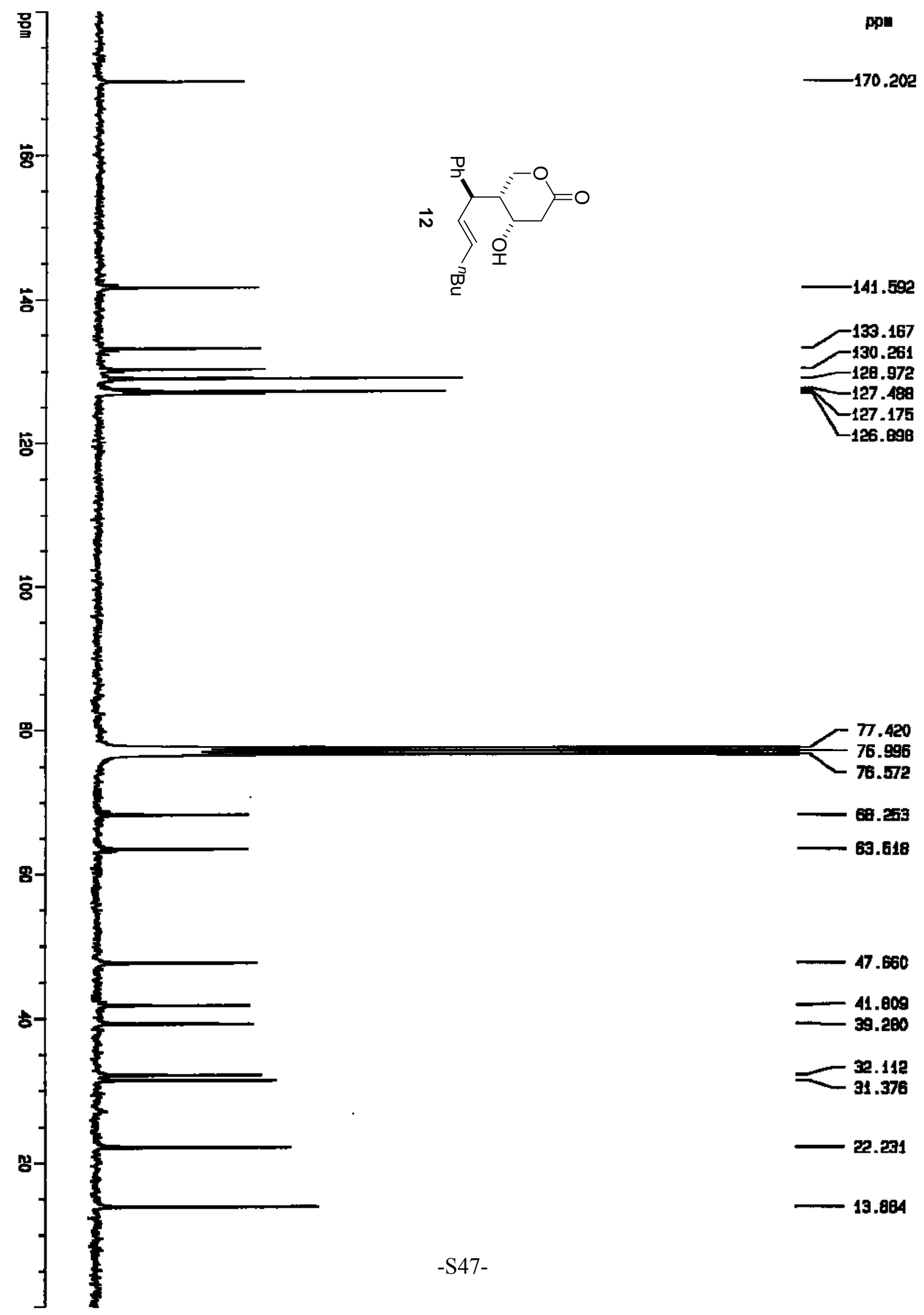



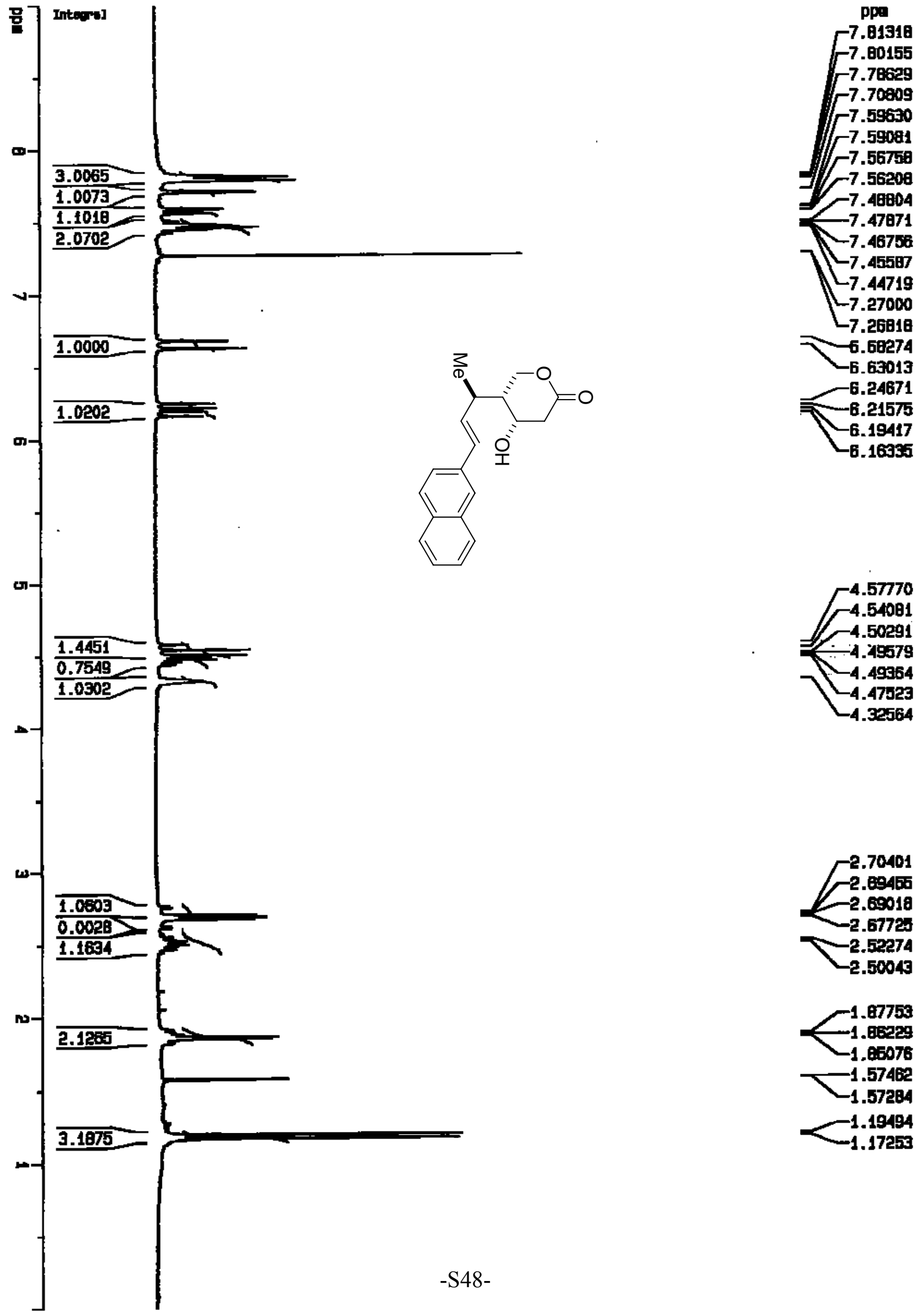


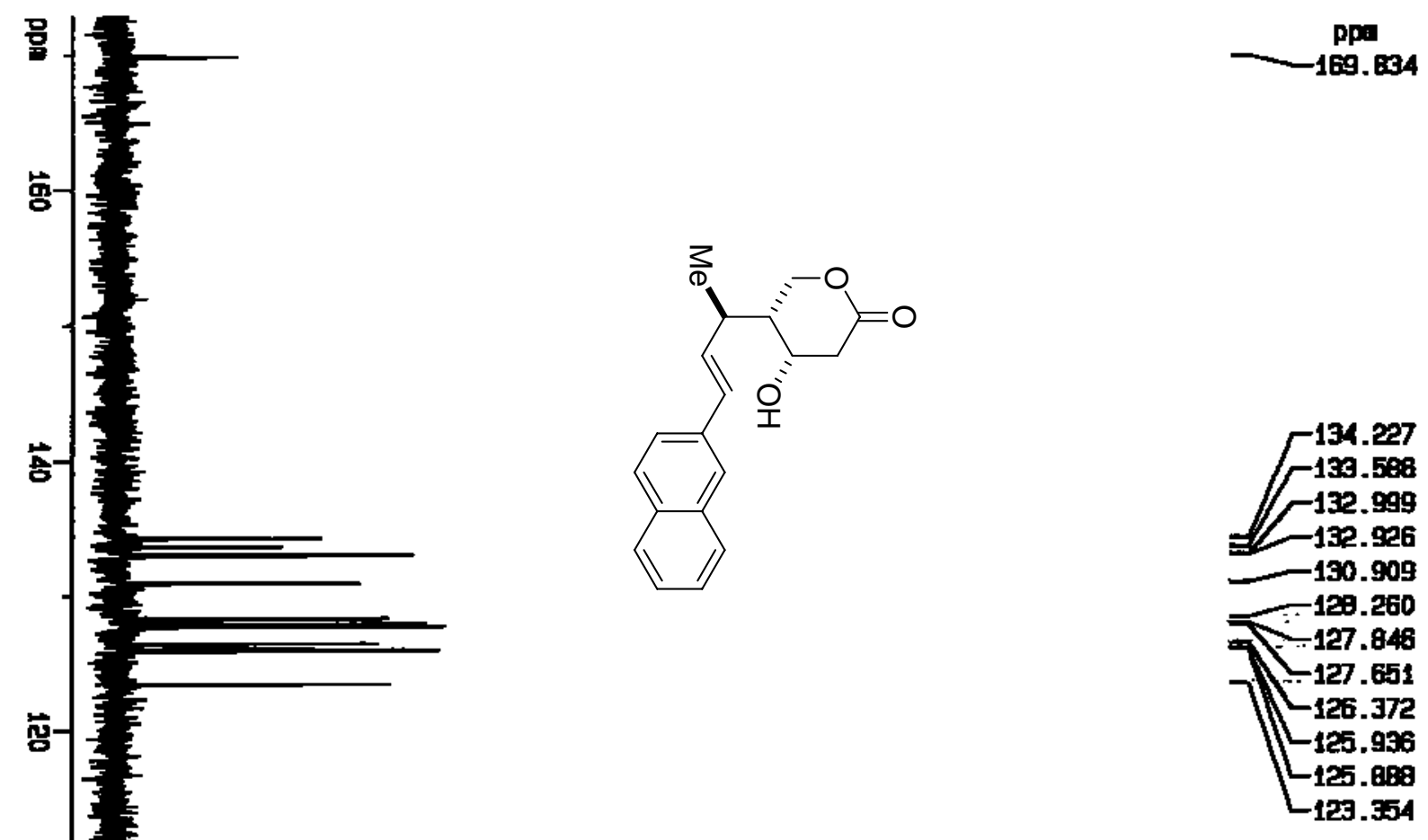

㝏
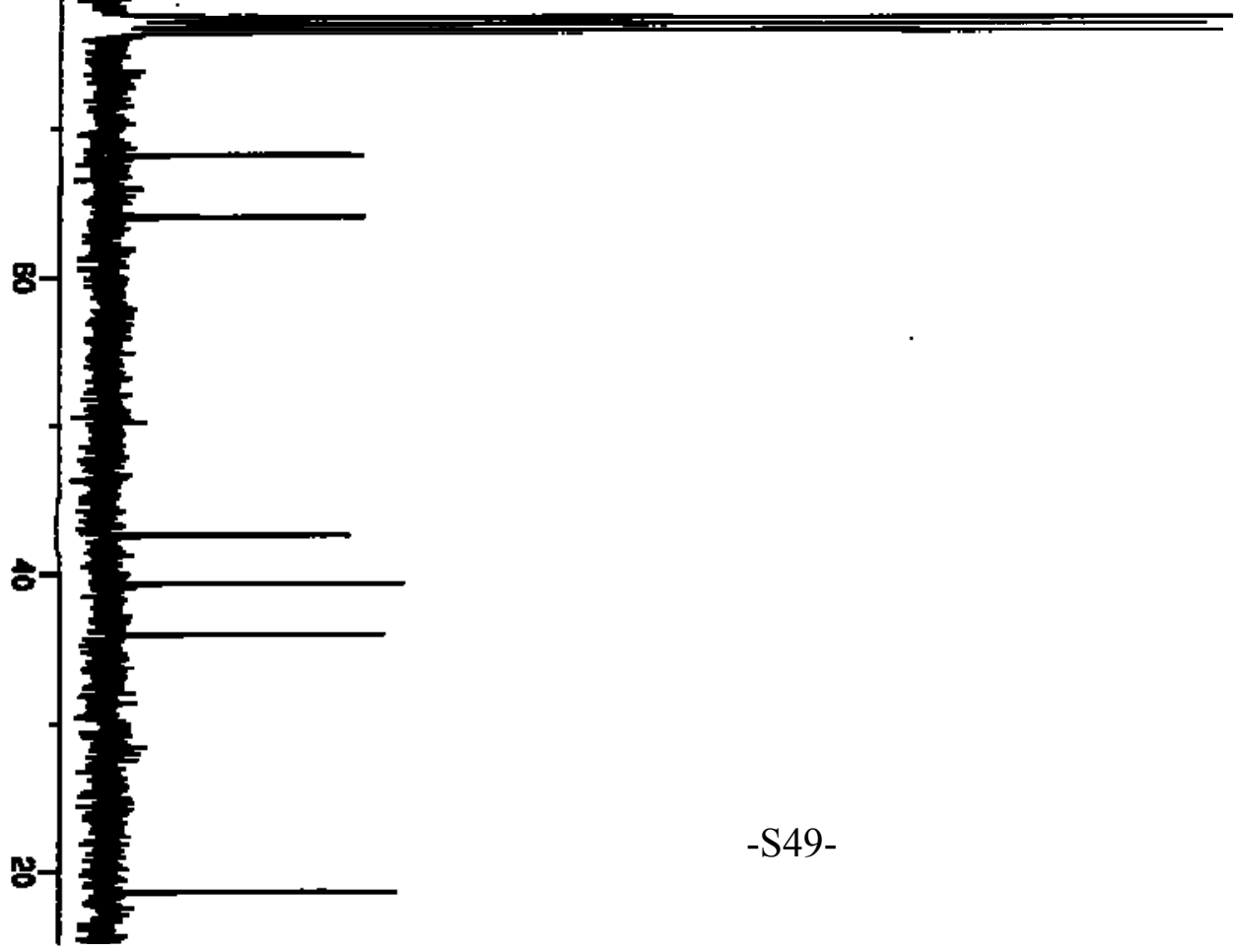


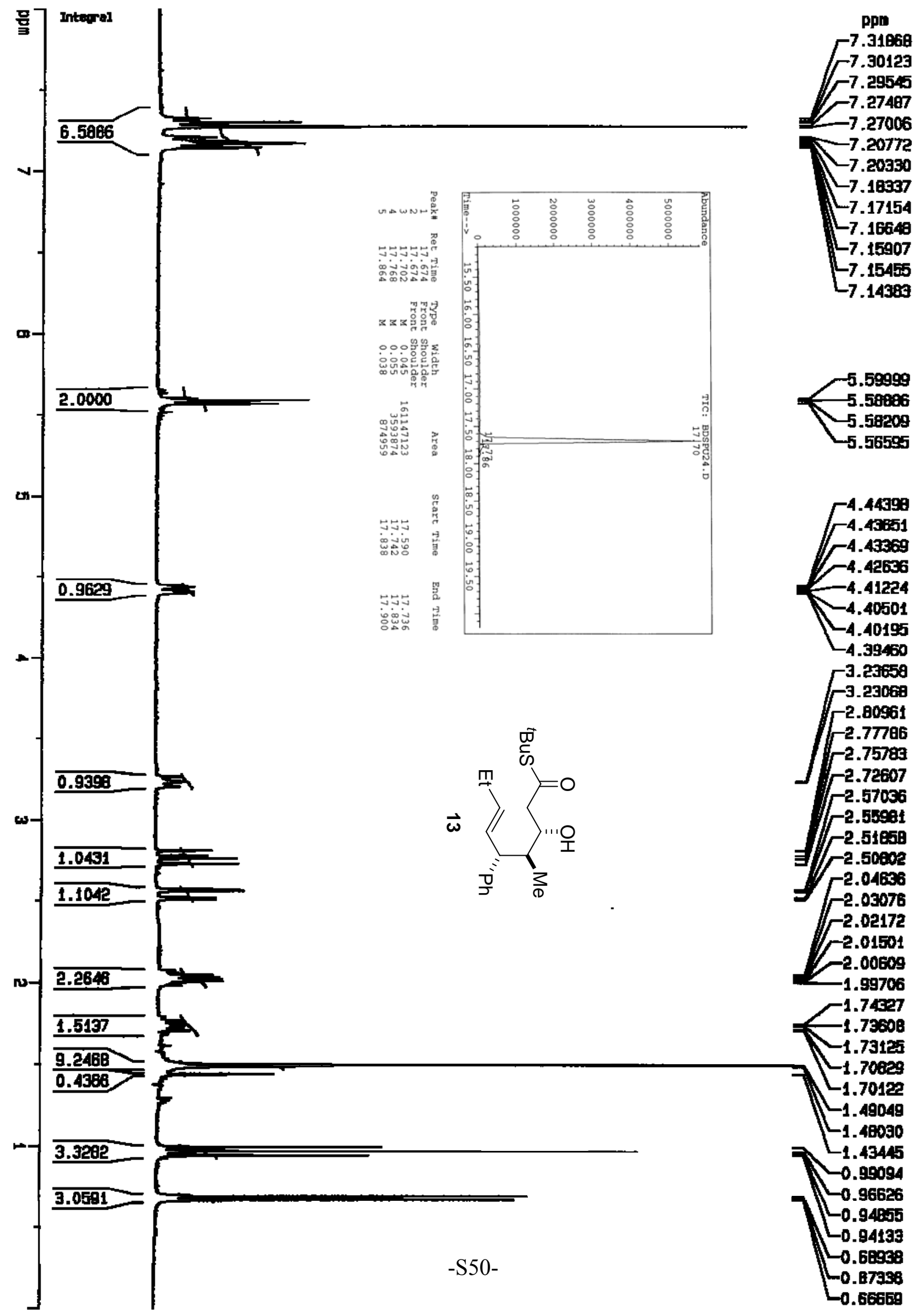



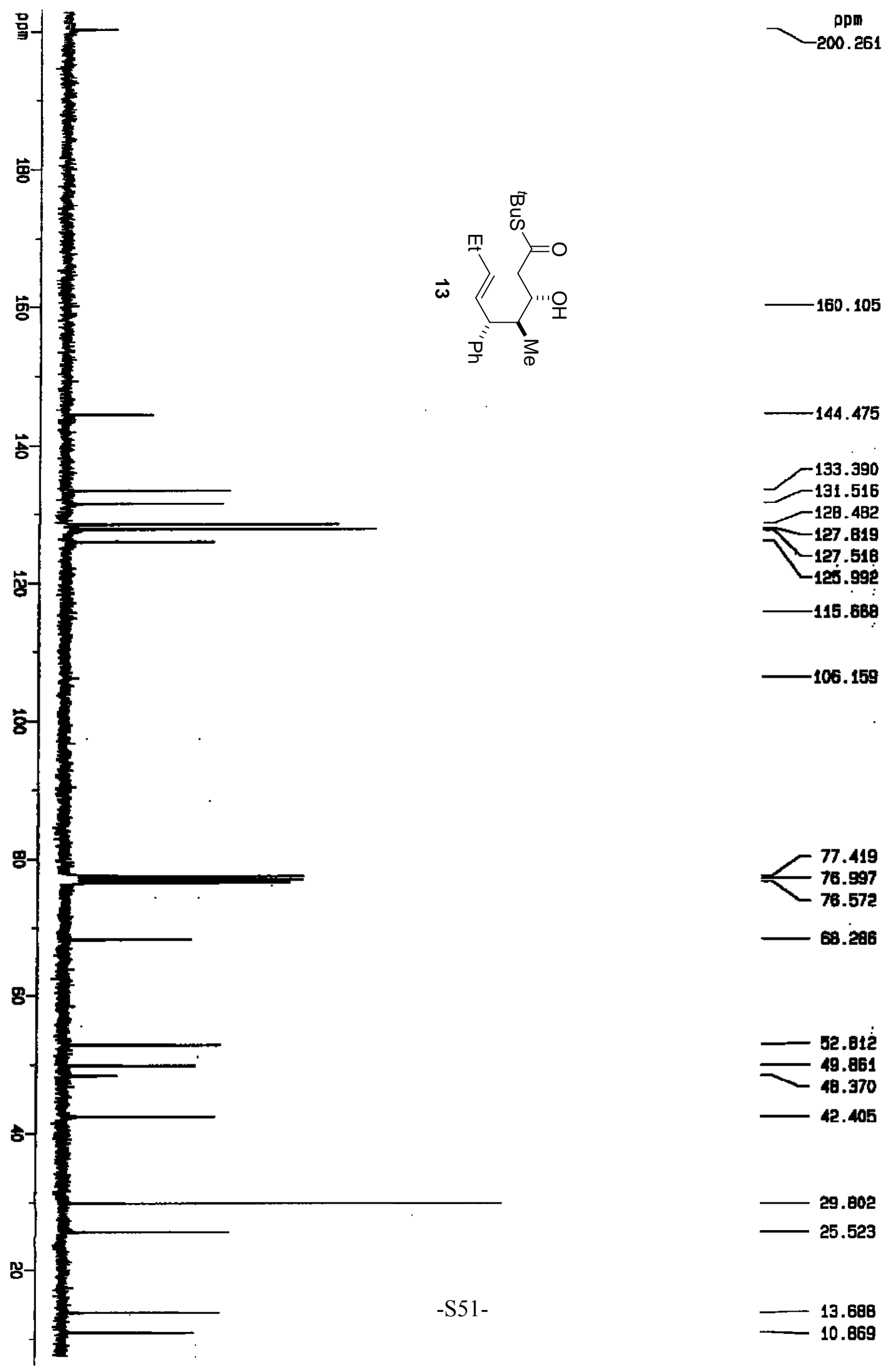

77.419

76.997

76.572

68.286

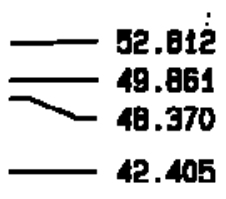

29.802

25.523 


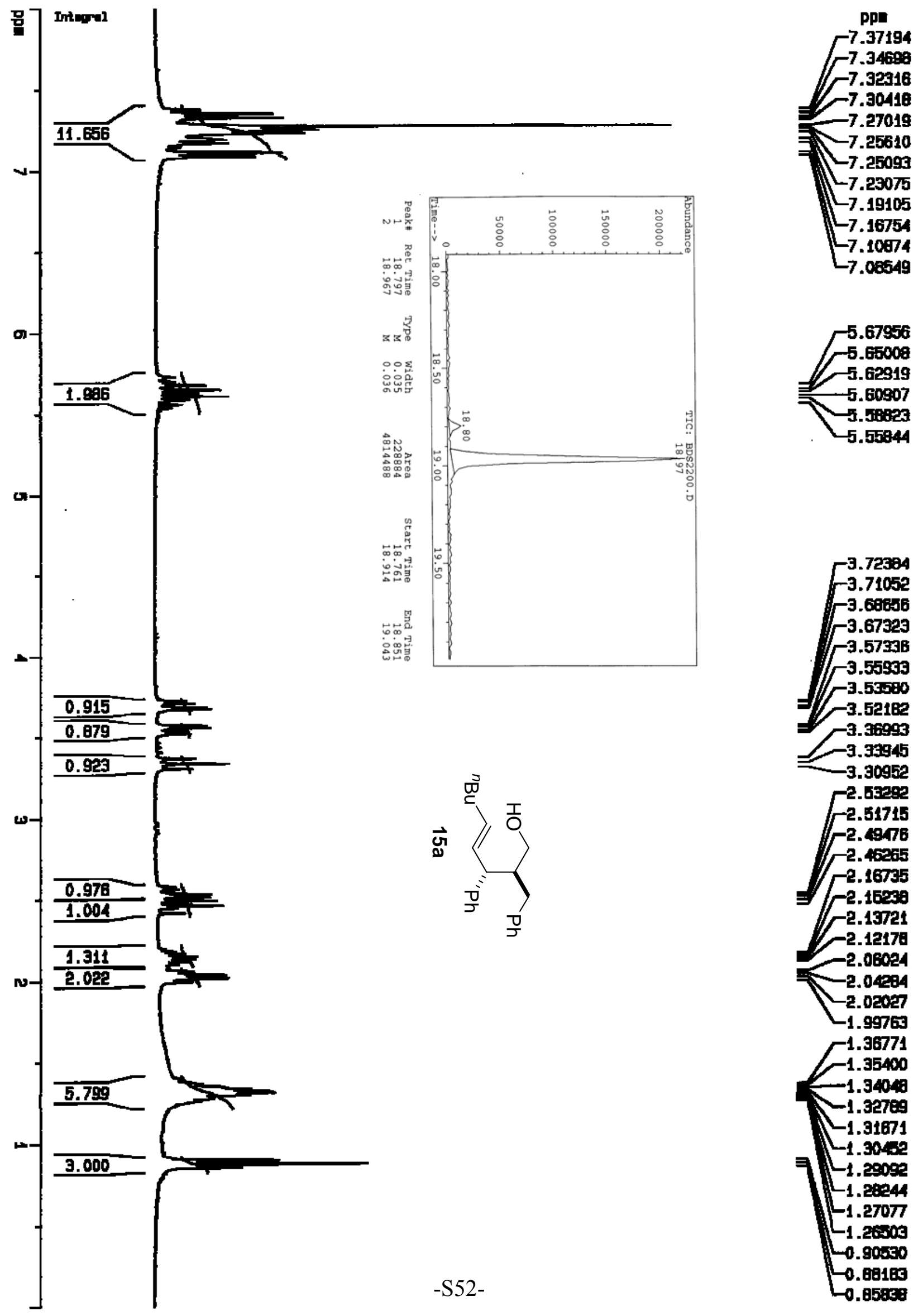




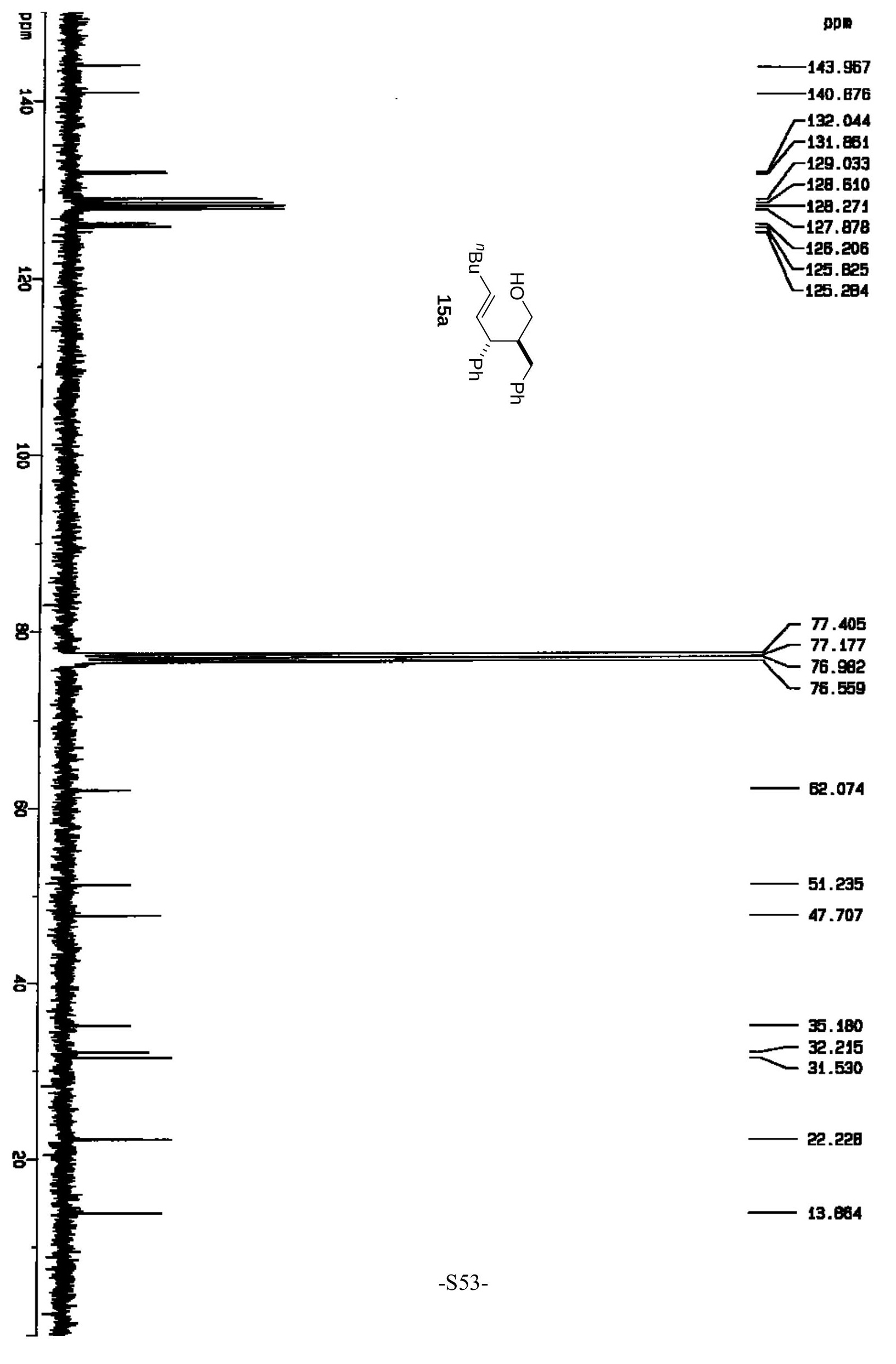




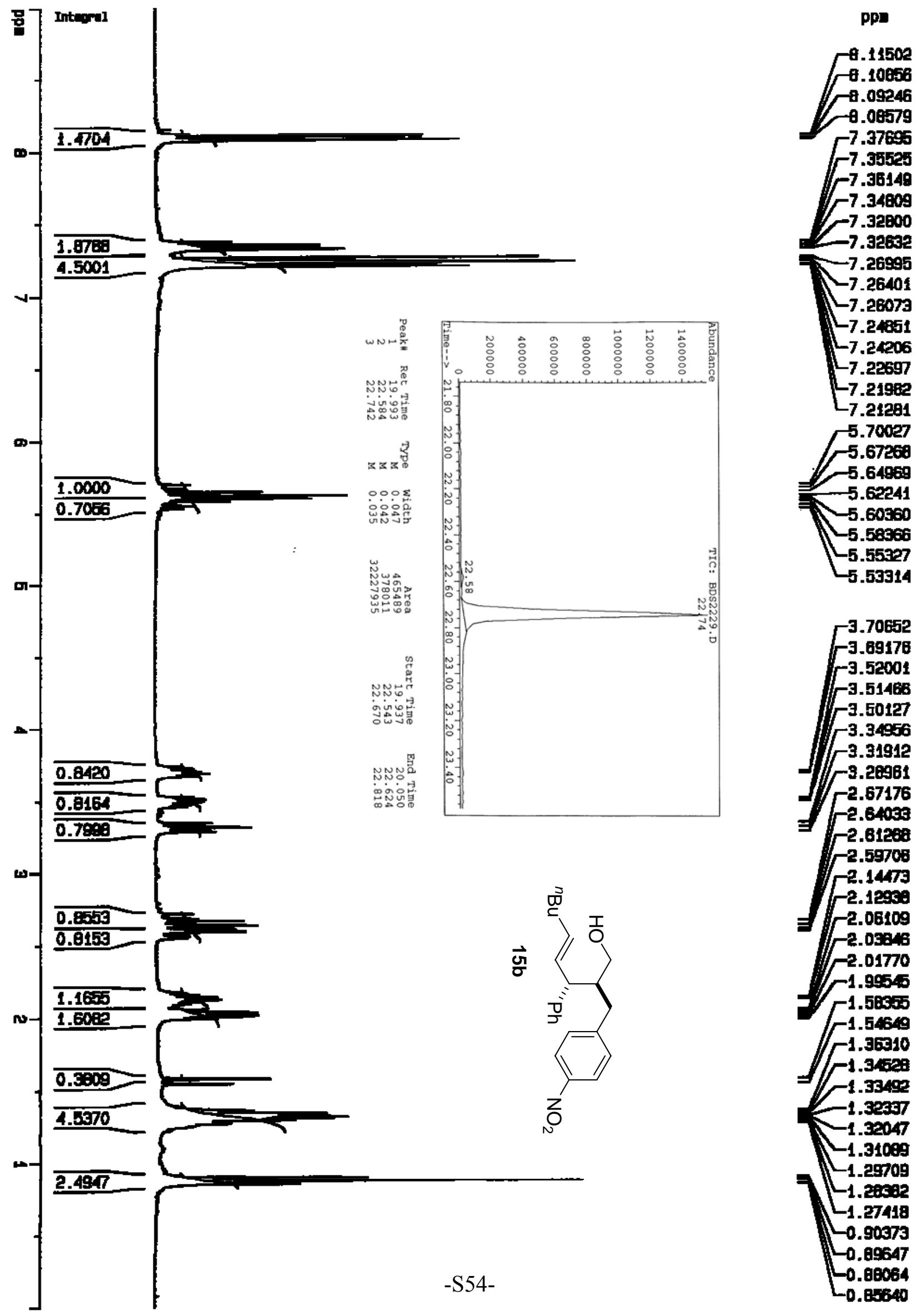




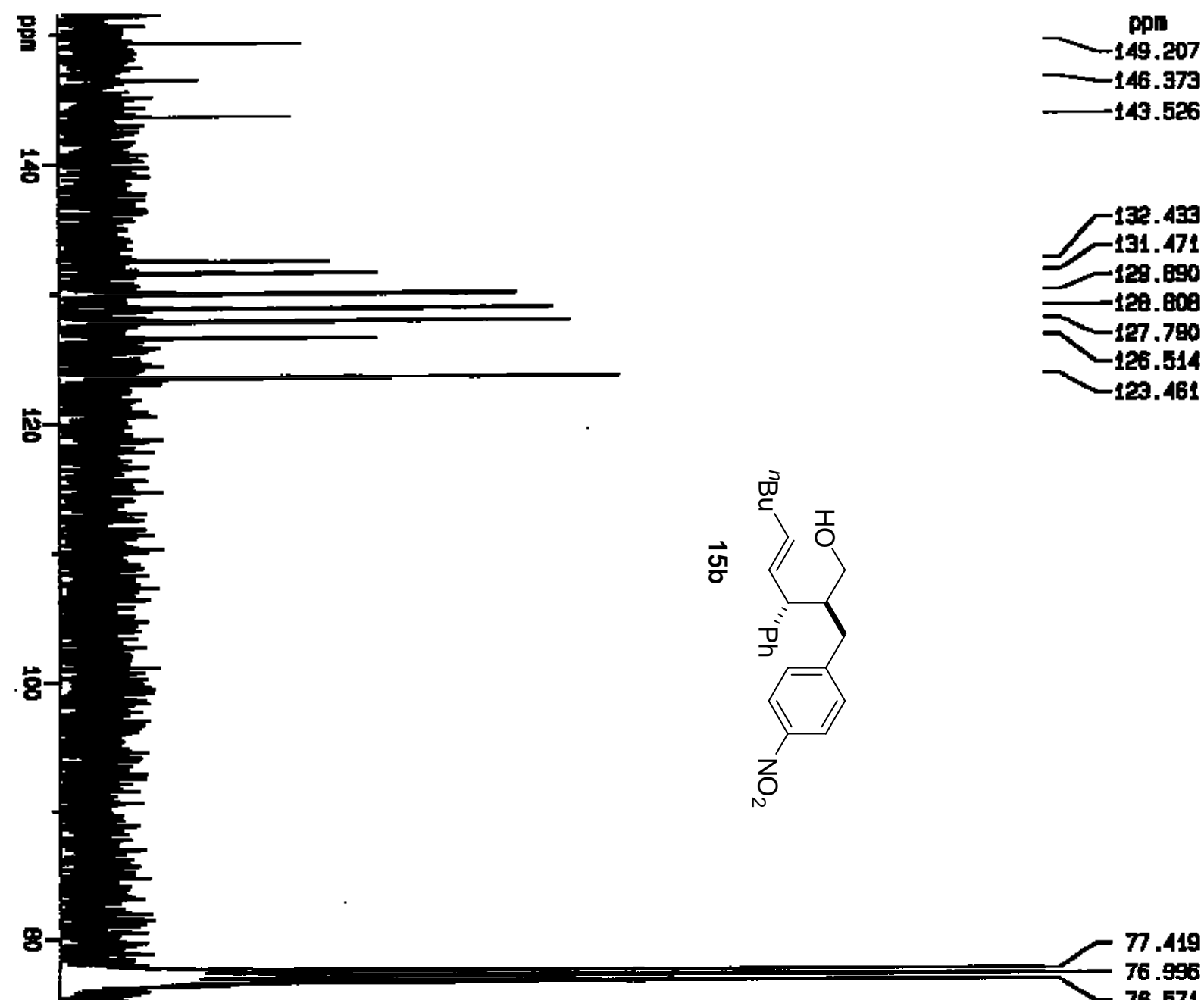

g
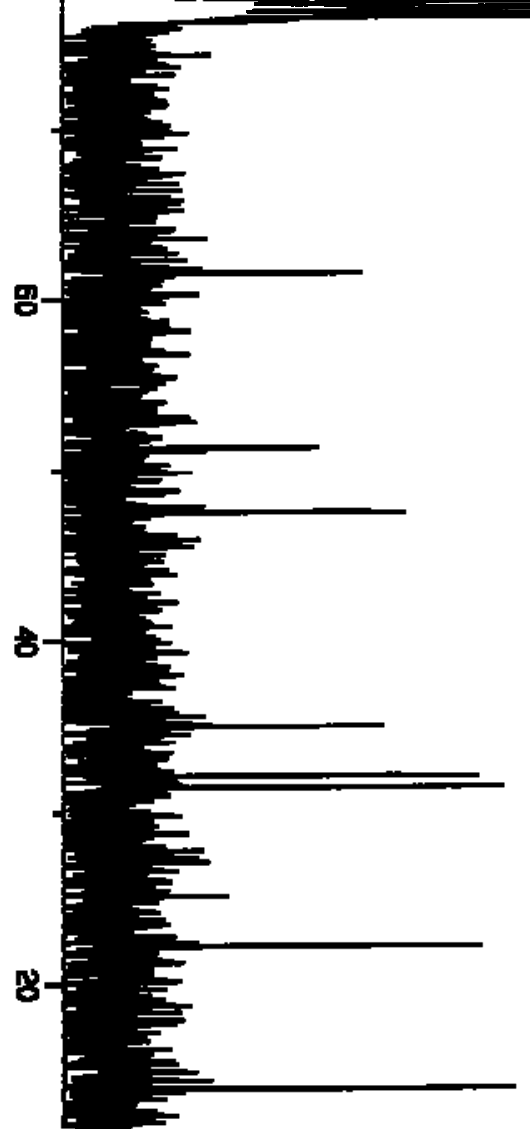


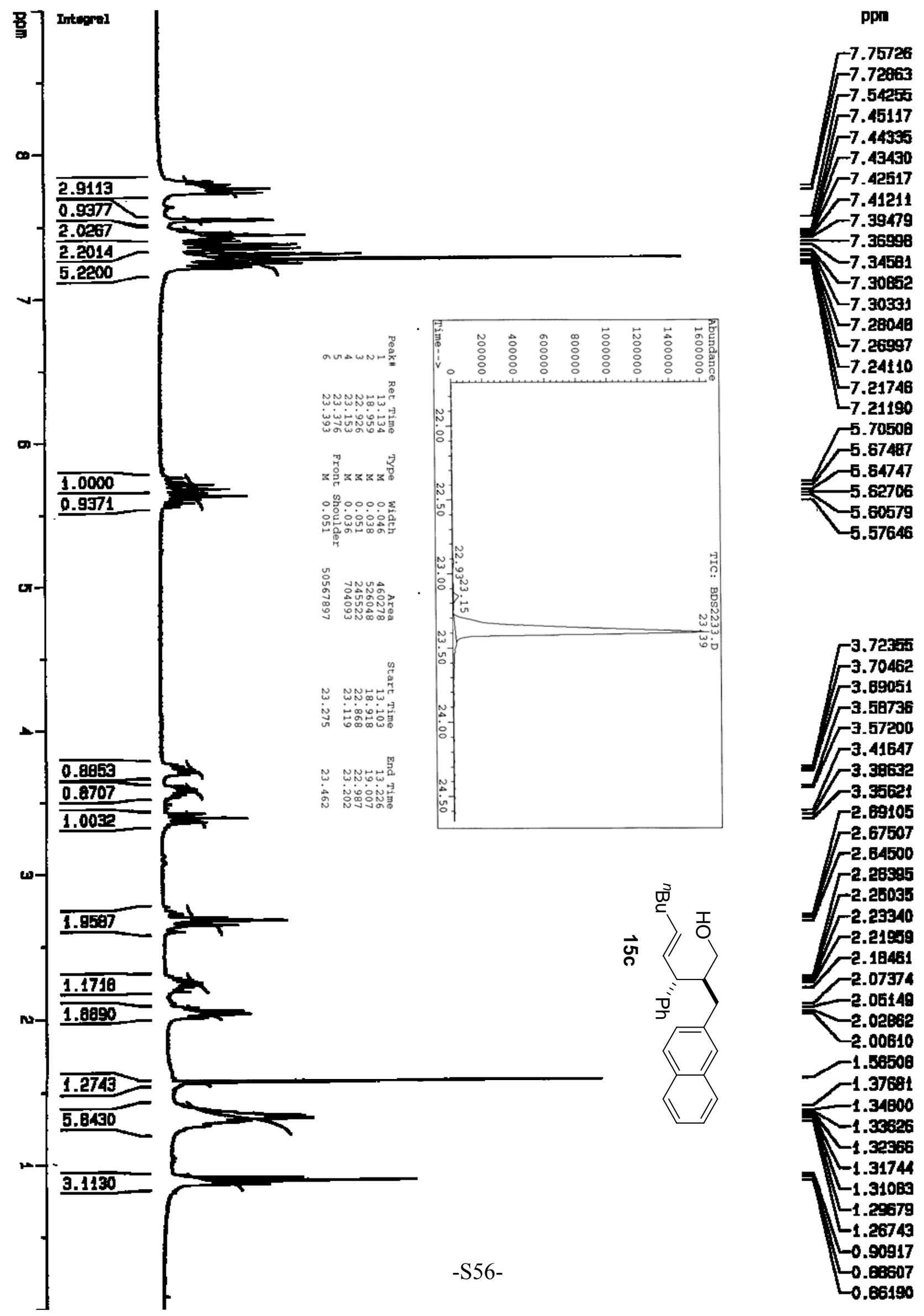



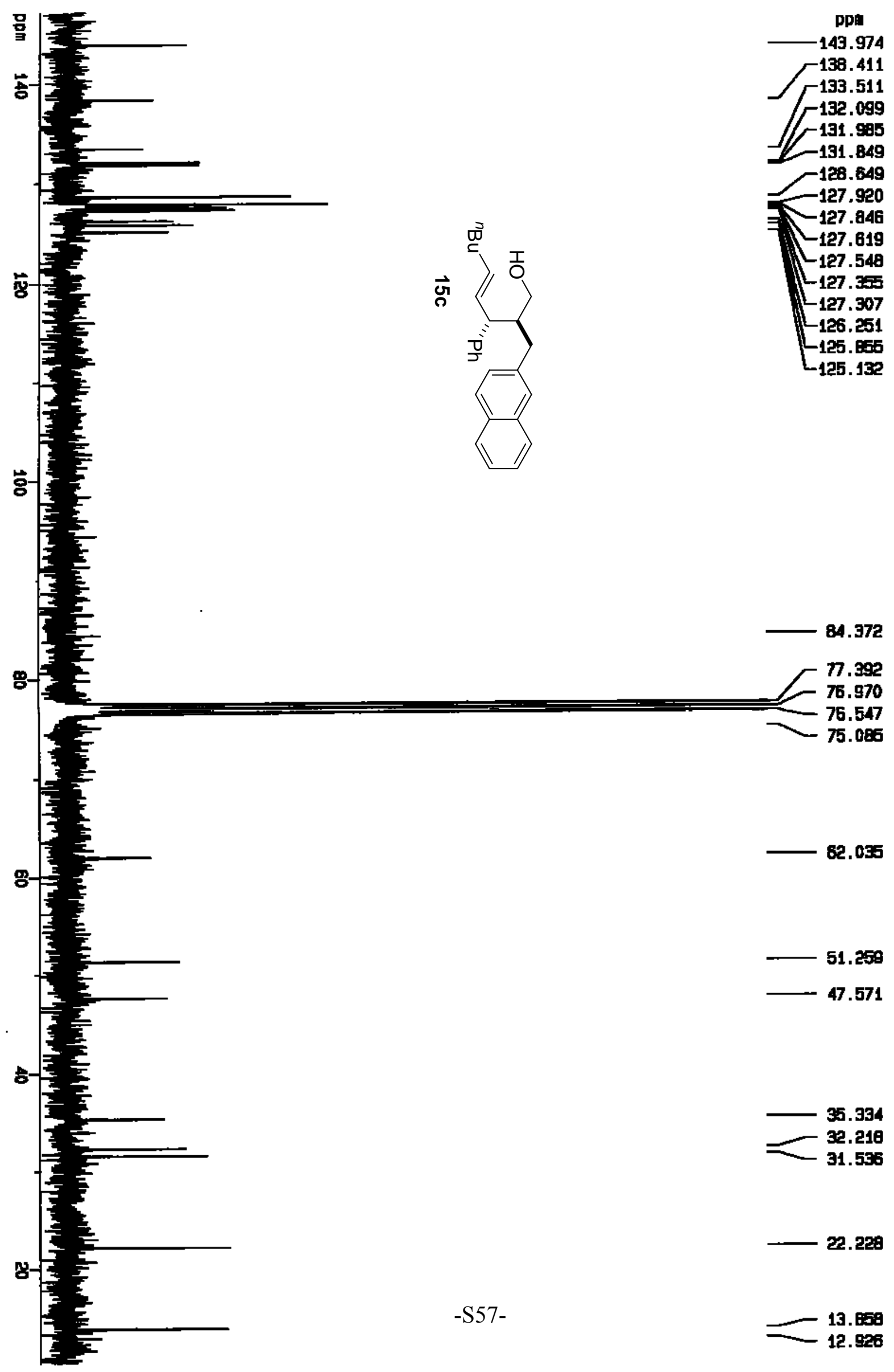

84.372

77.392

76.970

76.547

75.085

82.035

51.250

47.571

35.394

32.218

31.556

22.229 

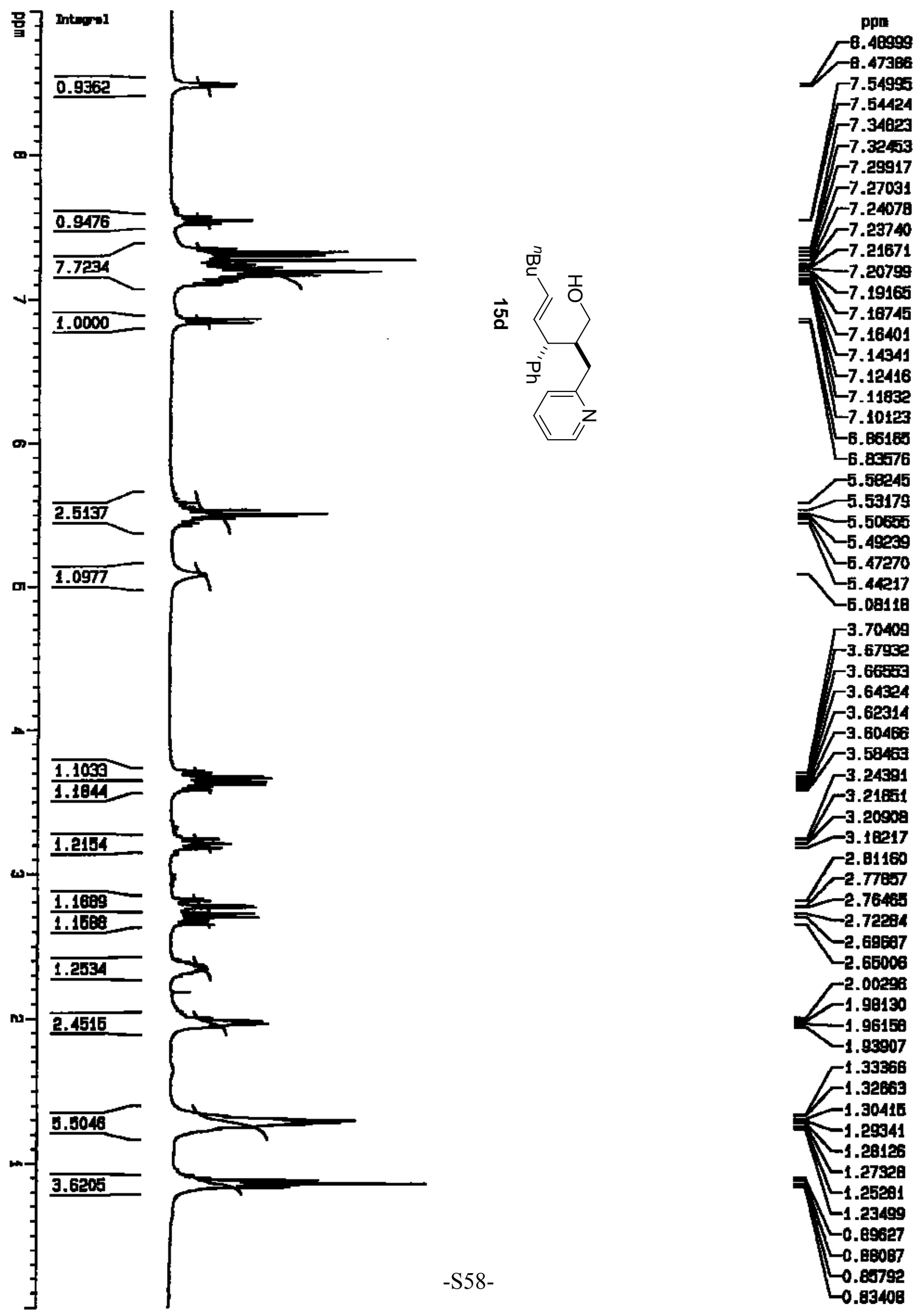

-2.81160

-2.77607
-2.76460

S-2.72284

$-2.69697$

$-2.65006$

$-2.00296$

$-1.98130$

$-1.96150$

$-1.95907$

1.33568
-1.32865
-1.30415
-1.29041
-1.28126

$=-1.27328$

- 1.25281

$L_{1.23499}$

- 0.69627 


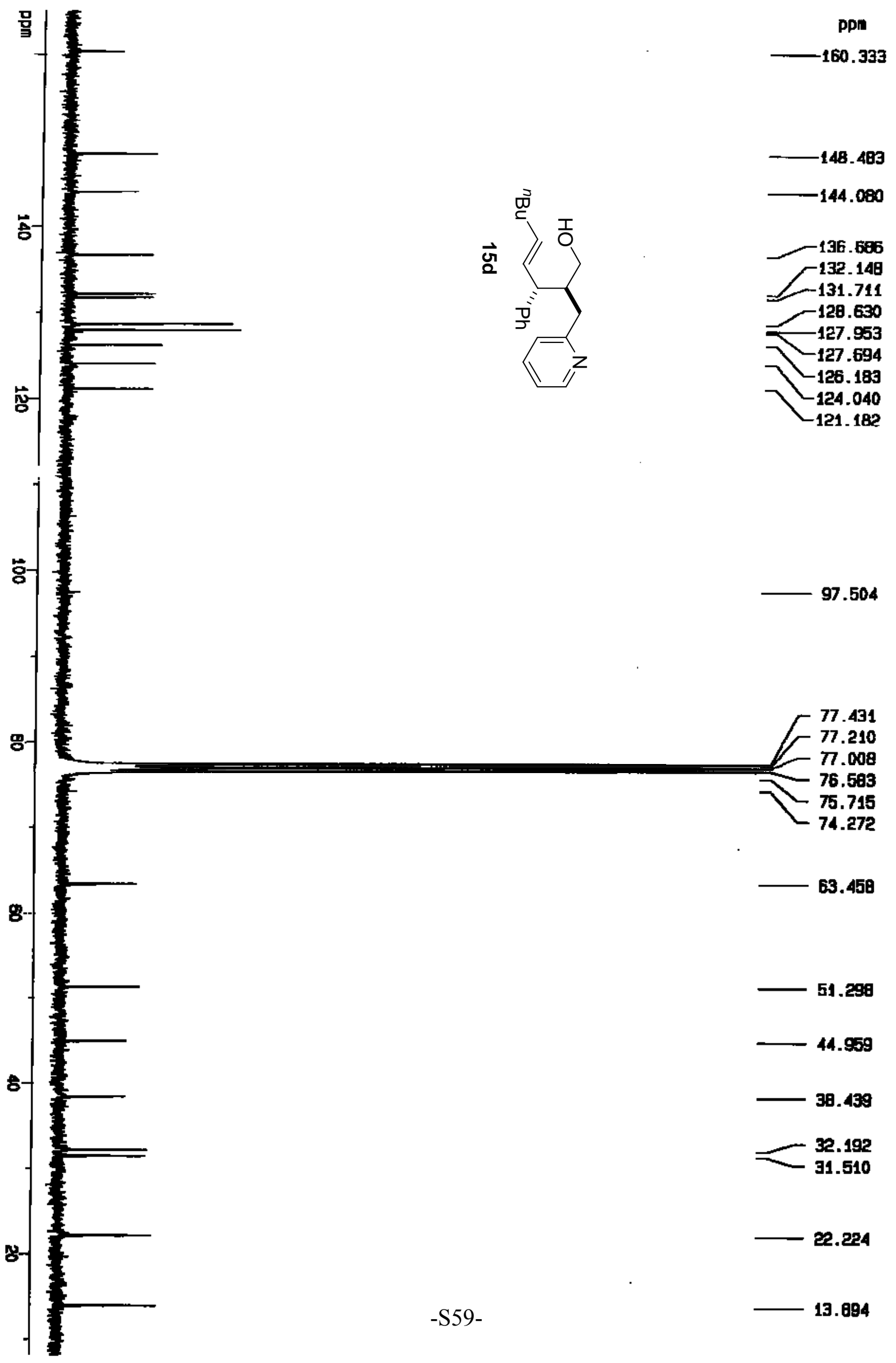



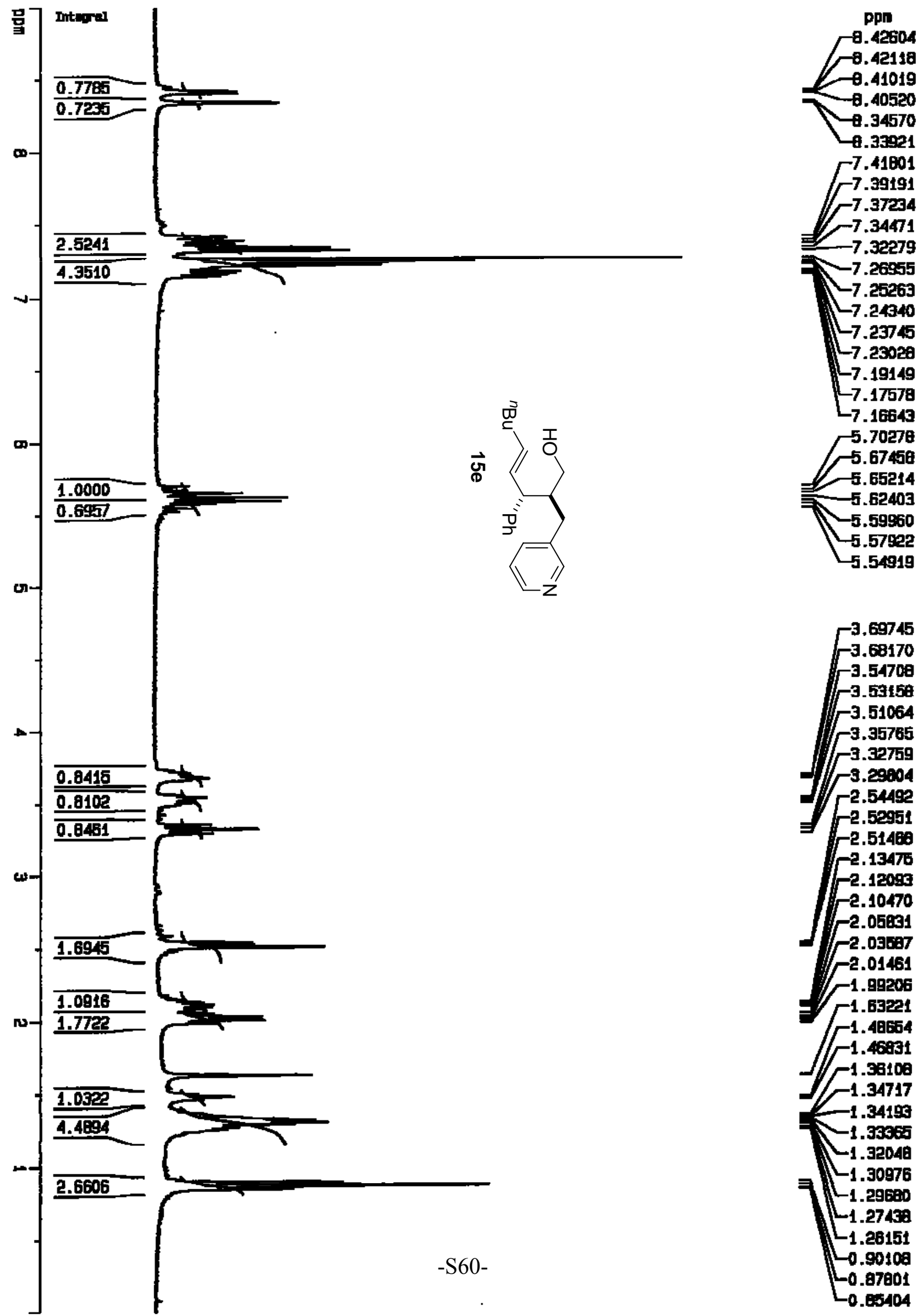

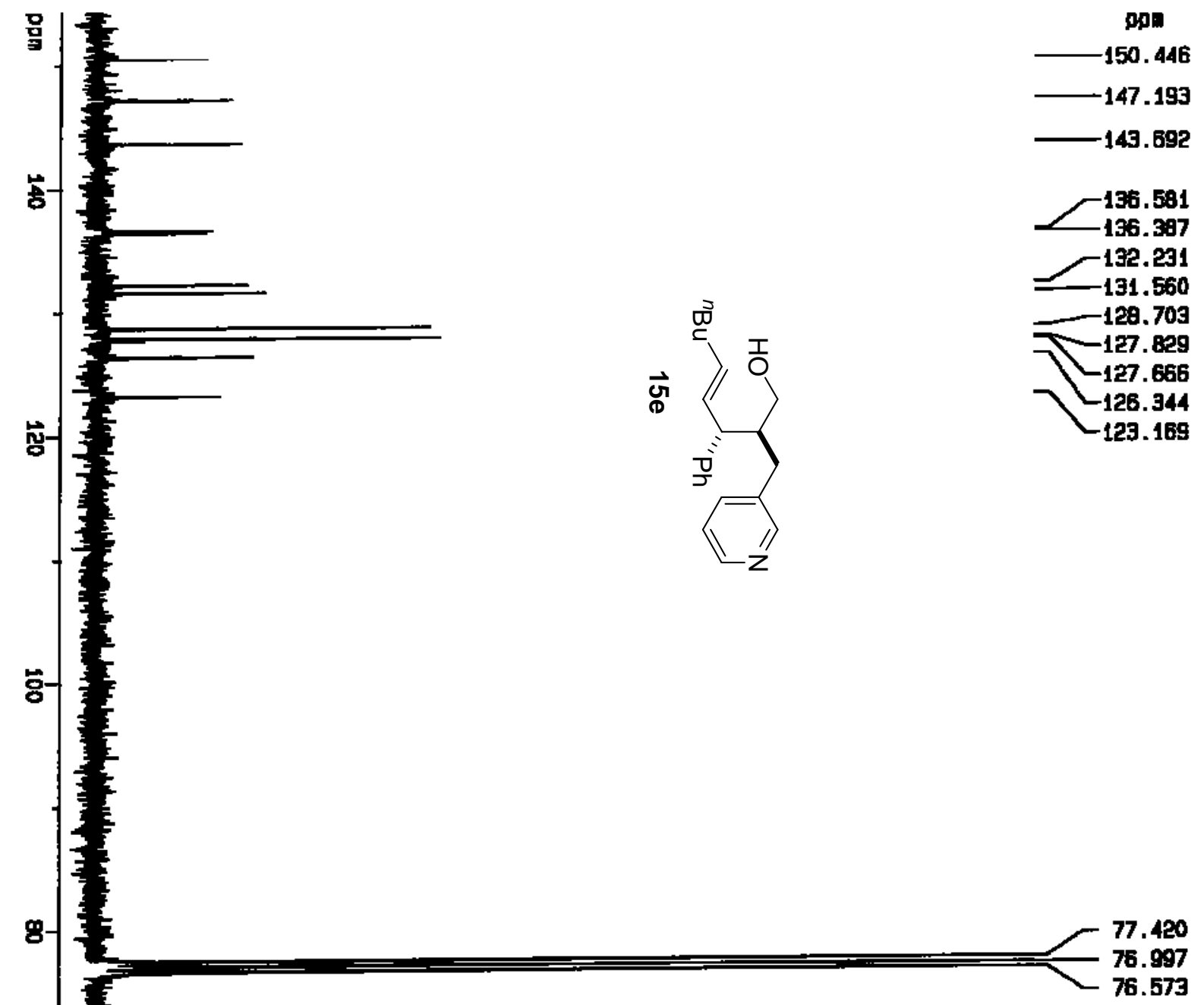

8

5-

8

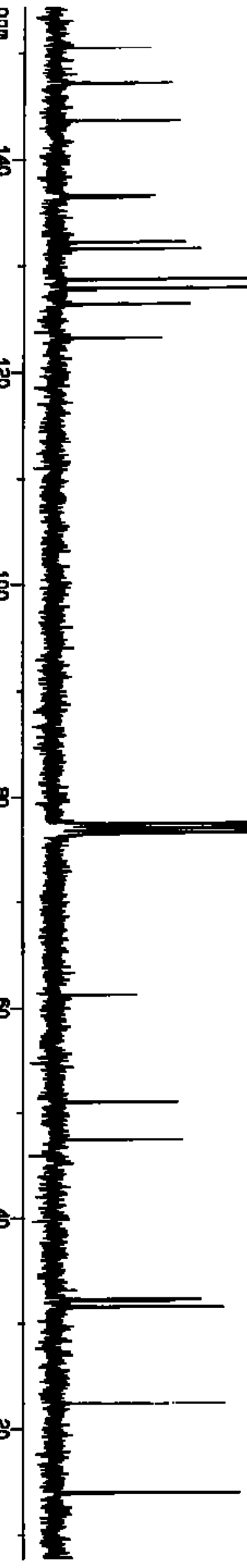

61.202

50.945

47.328

32.191

32.036

31.489

22.213 

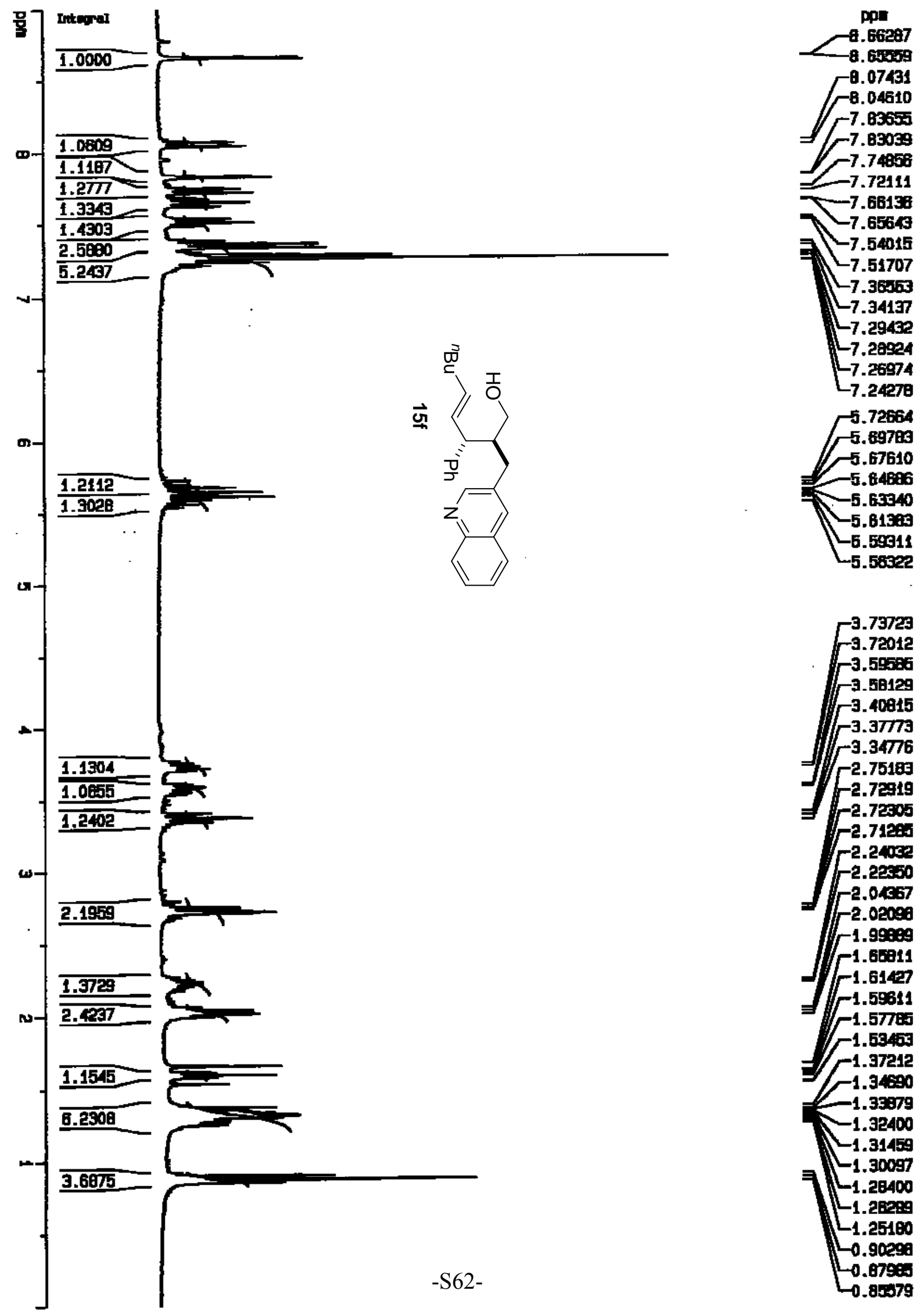

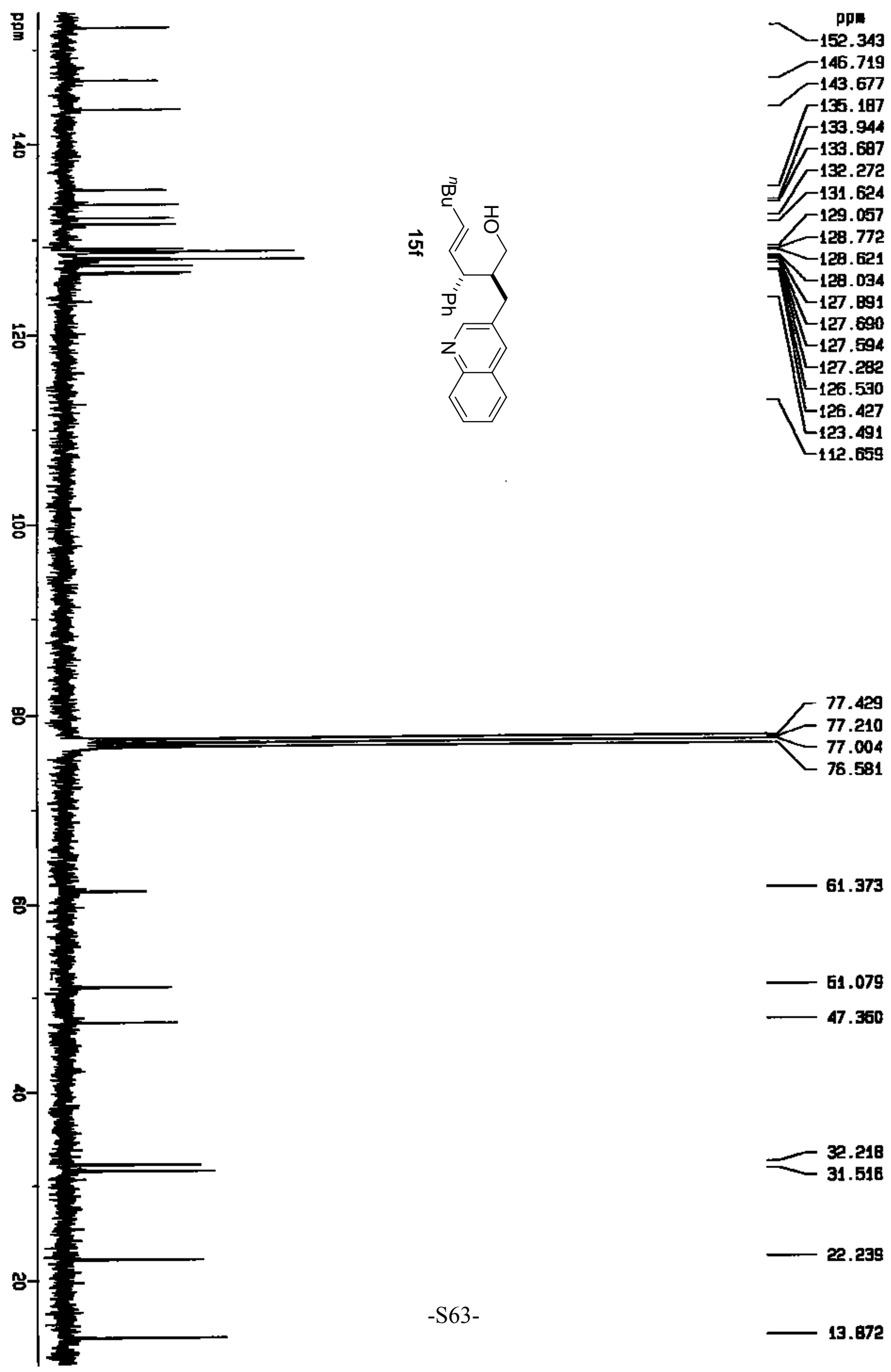

77.429

77.210

77.004

76.581

61.373

61.079

47.350

32.218

31.516

22.239 


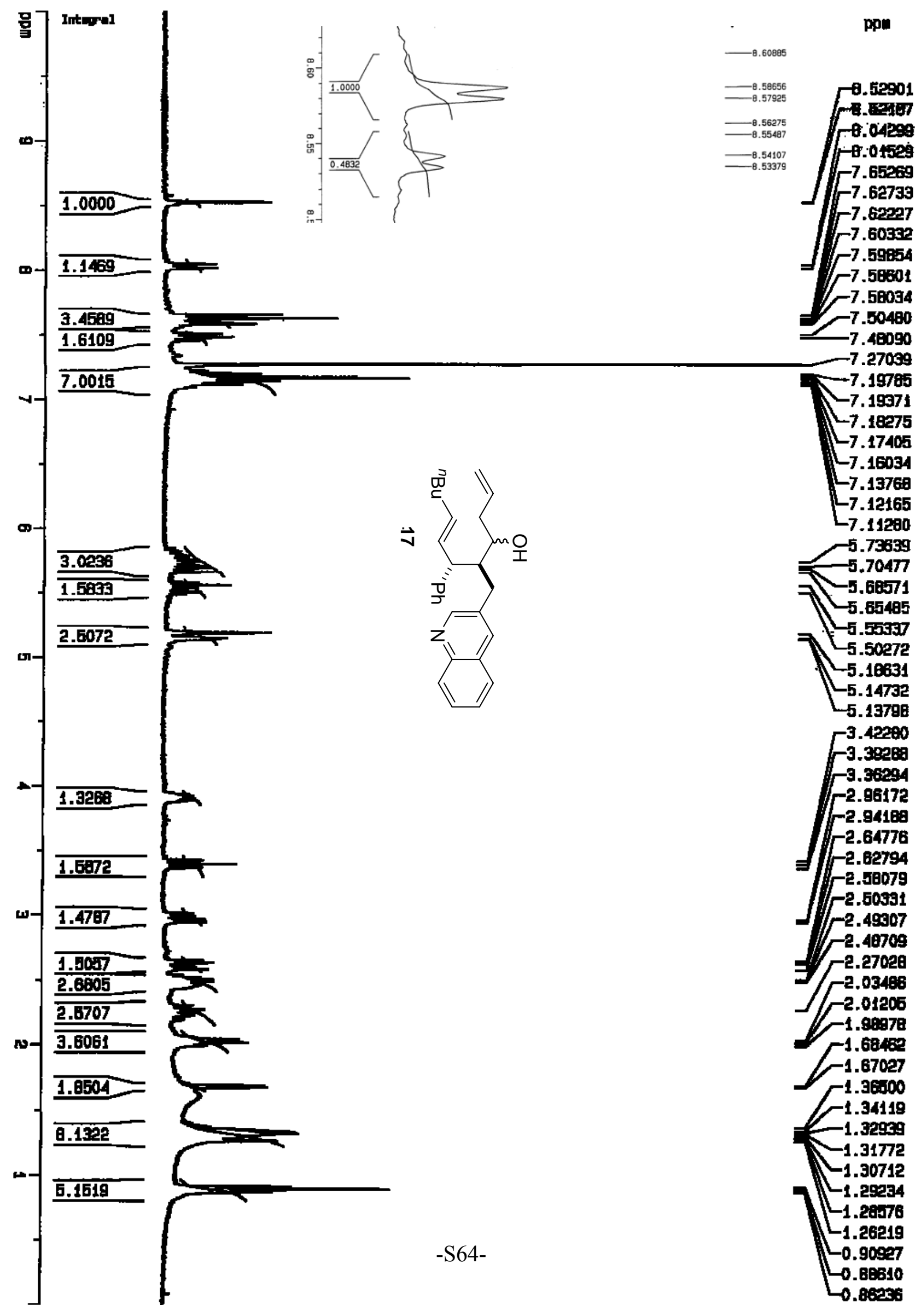




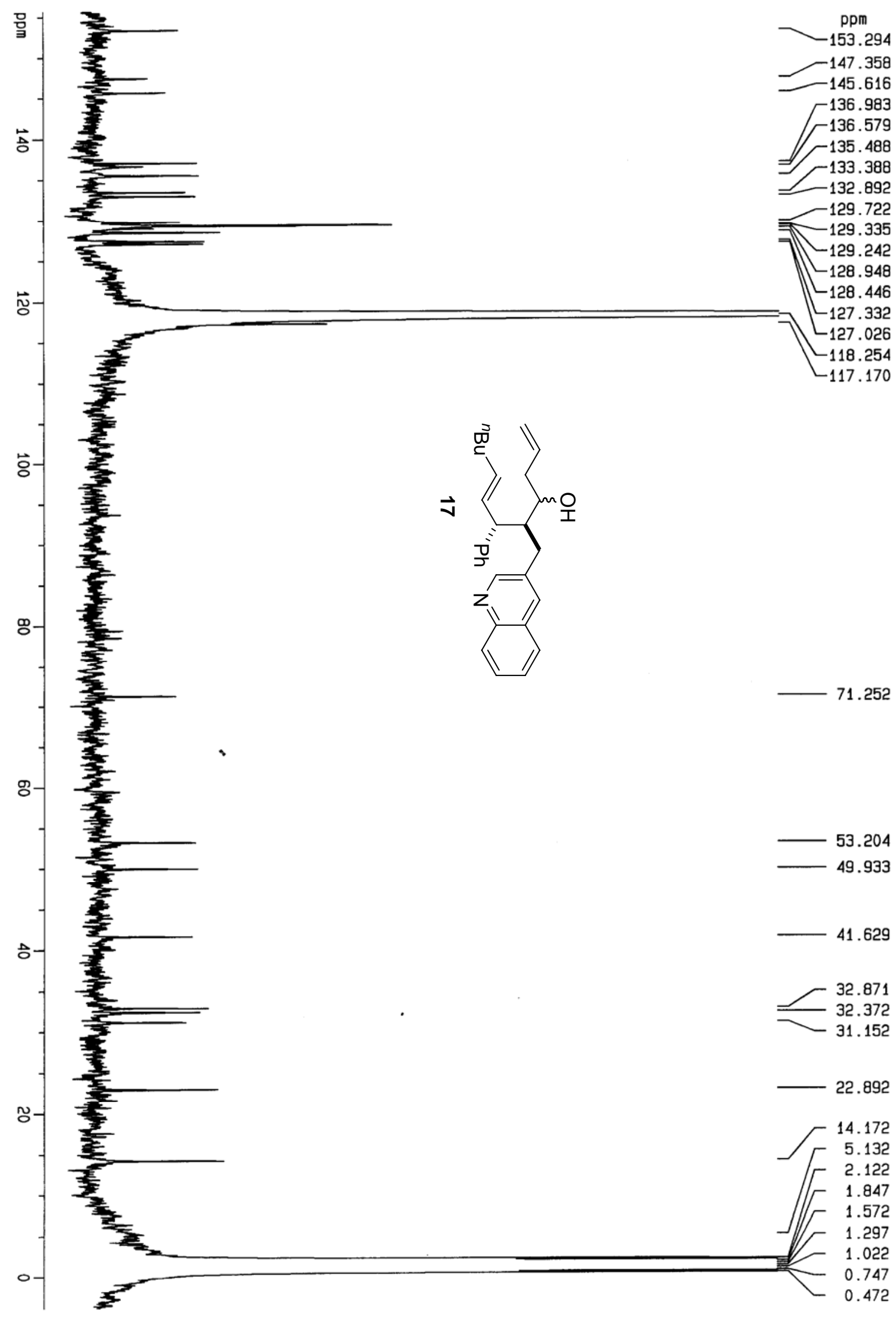



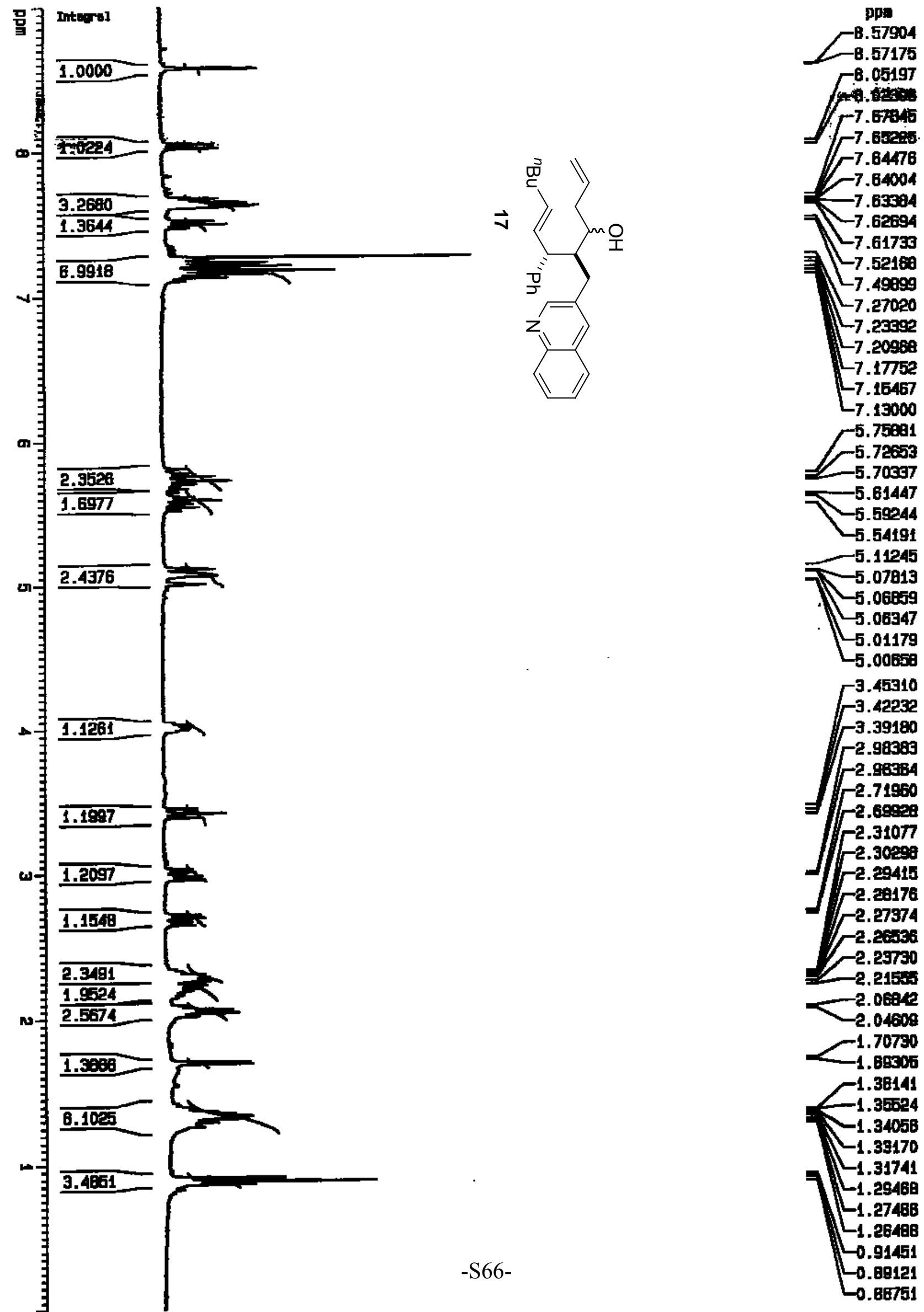

$-1.70730$

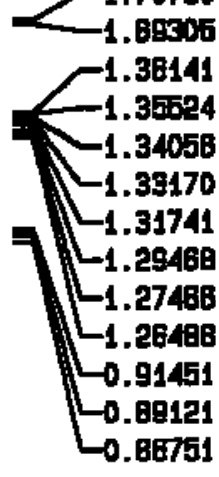




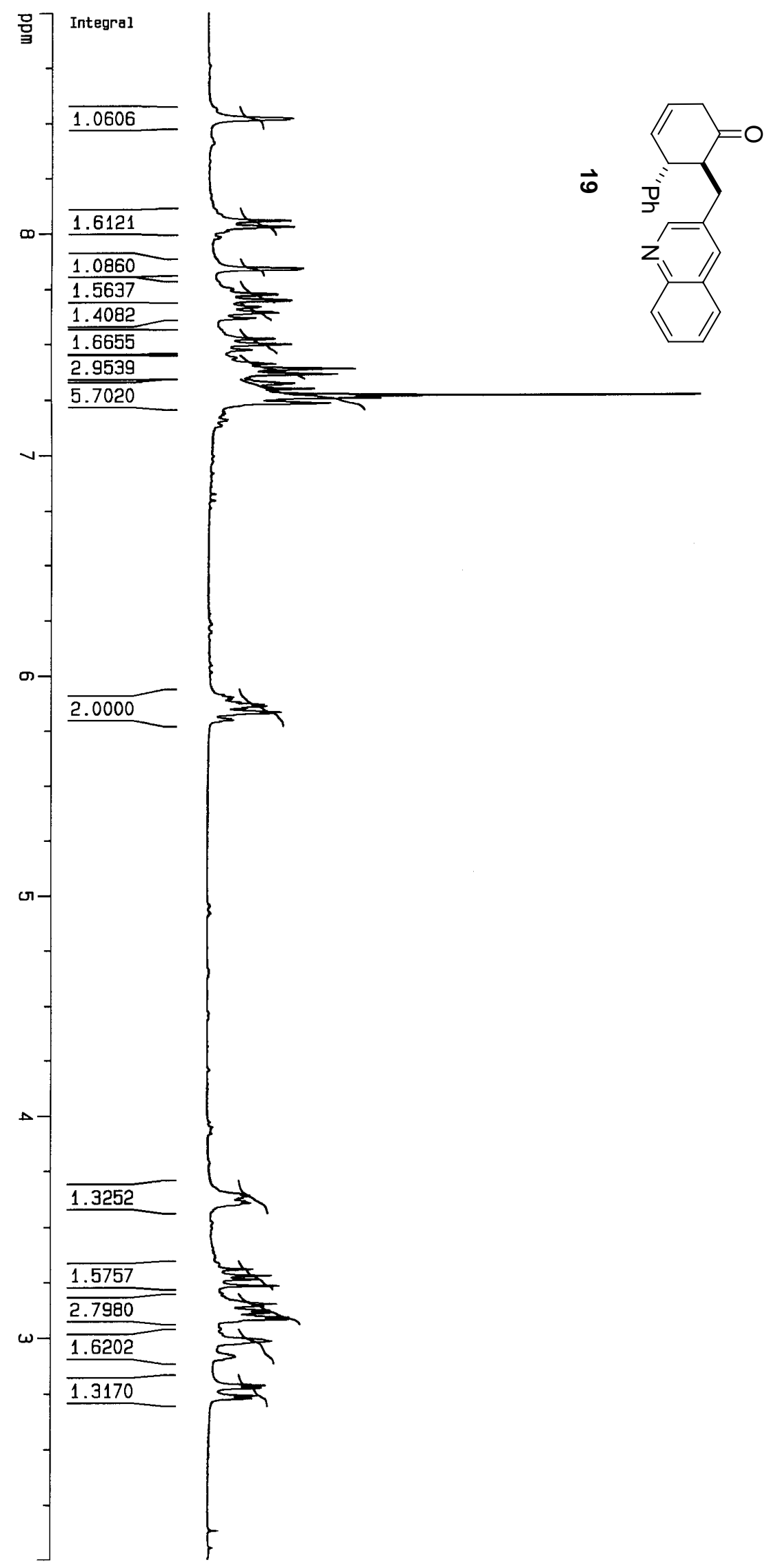

$\mathrm{ppm}$
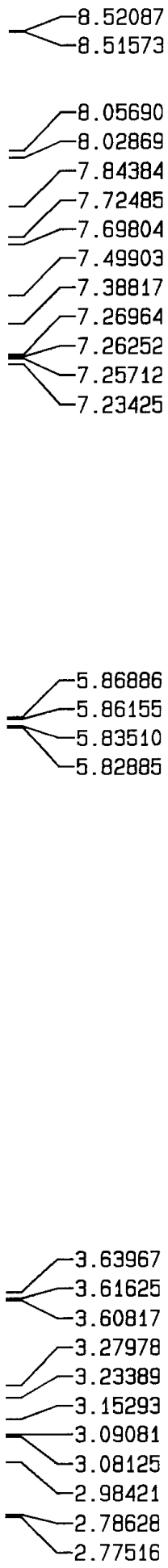

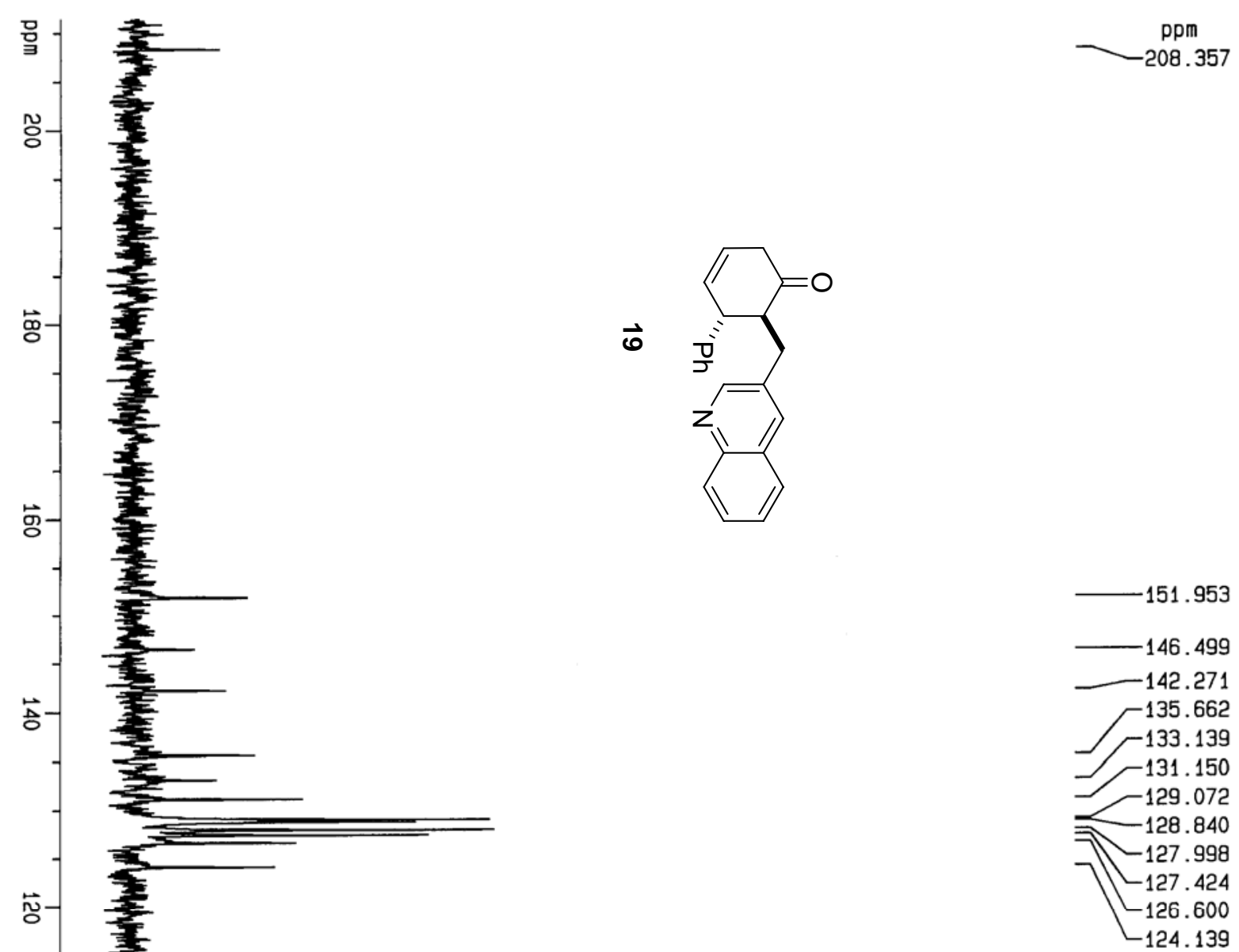

安

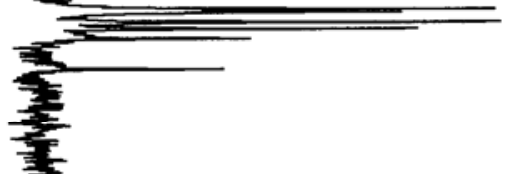

77.419

迢

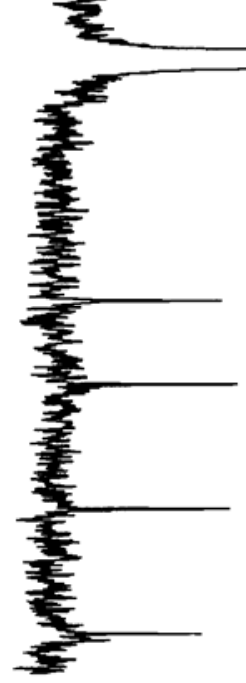

